\title{
On energy minimizers of the diblock copolymer problem
}

\author{
XIAOFENG REN ${ }^{\dagger}$ \\ Department of Mathematics and Statistics, Utah State University, \\ Logan, UT 84322, U.S.A. \\ AND \\ JUNCHENG WEI \\ Department of Mathematics, The Chinese University of Hong Kong, \\ Shatin, Hong Kong
}

[Received 17 July 2002 and in revised form 24 January 2003]

\begin{abstract}
We view the free energy of a diblock copolymer system as a variational problem, in which the integrand of the functional contains an interesting nonlocal term, and a small parameter $\epsilon$. We prove that as $\epsilon$ approaches 0 , the energy minimizers develop a growing number, of order $\epsilon^{-1 / 3}$, of periodic oscillations, explaining the micro-phase separation phenomenon.
\end{abstract}

\section{Introduction}

A di-block copolymer molecule is a linear chain consisting of two subchains $a$ and $b$ grafted covalently to each other. The subchains $a$ and $b$ are made of different monomer units $A$ and $B$, respectively. In polymer systems even a weak repulsion between unlike monomers $A$ and $B$ induces a strong repulsion between $a$ and $b$. As a result the different subchains tend to segregate below some temperature $T_{c}$, but as they are chemically bonded, even a complete segregation of subchains $a$ and $b$ cannot lead to a macroscopic phase separation. Only a local micro-phase separation occurs: micro-domains rich in $A$ and $B$ are formed.

In [12] Ohta and Kawasaki introduced a free energy functional

$$
\mathcal{F}(u)=\int_{\Omega}\left[\frac{\epsilon^{2}}{2}|\nabla u|^{2}+W(u)+\frac{\sigma}{2}\left|(-\Delta)^{-1 / 2}(u-m)\right|^{2}\right] \mathrm{d} x .
$$

The original formula in [12] is given for the whole space. The expression here on a bounded domain first appeared in Nishiura and Ohnishi [10].

The two unlike monomer units are represented by $u=-1$ and $u=1$ respectively. The connectivity of the monomers in a chain leads to the long range interaction $(\sigma / 2)\left|(-\Delta)^{-1 / 2}(u-m)\right|^{2}$ in the free energy. Here $-\Delta$ is viewed as a positive operator, and $(-\Delta)^{-1 / 2}$ is the square root of its inverse. The parameter $\sigma$ is proportional to the inverse of the square root of the total chain length of the copolymer. $\left(\epsilon^{2} / 2\right)|\nabla u|^{2}$ represents the interfacial energy density at bonding points. The parameter $\epsilon$ is proportional to the thickness of interfaces between the two monomers. $m$ stands for the mass ratio of the two monomer units.

\footnotetext{
† Supported in part by NSF grant DMS-9703727. Email: ren@math.usu.edu

${ }^{\ddagger}$ Supported in part by an Earmarked Grant of RGC of Hong Kong. Email: wei@ math.cuhk.edu.hk
} 
When this free energy is minimized, the first term of the integrand prefers large blocks of monomers, thereby reducing the combined size of interfaces between the two monomers. The function $W$ in the second term is a double-well potential with two global minima at -1 and 1 , reflecting its preference for segregated monomers over mixtures. The third term, most interesting to us, depends on $u$ nonlocally, through a global operator $(-\Delta)^{-1 / 2}$. It favors rapid oscillation between the two monomers. When all these factors compete, the phenomenon known as microphase separation occurs.

The one-dimensional case $\Omega=(0,1)$ is particularly interesting because of the laminar structures observed in diblock copolymers. In an earlier paper [13] we studied the parameter range $\sigma \sim \epsilon$. Physically this means that the size of the sample is of order $N^{2 / 3} l$ where $N$, the polymerization index, is the number of monomers in a chain molecule and $l$ is the average distance between two adjacent monomers. We proved the existence of a family of local minima when $\epsilon$ is small, which are nearly periodic with the sizes of periods comparable to the size of the domain $(0,1)$.

In this paper we study a different parameter range $\sigma \sim 1$. Physically we are taking a larger sample of size $N l$. The admissible set is

$$
X_{m}=\left\{u \in W^{1,2}(0,1): \int_{0}^{1} u(x) \mathrm{d} x=m\right\}, \quad m \in(-1,1) .
$$

The constraint $\int_{0}^{1} u=m$ reflects the total mass of one of the two micro-components. It must be in $(-1,1)$ in order to have a mix of the two monomer units $(u=-1$ and $u=1$ respectively).

We restate the functional as

$$
I_{\epsilon}(u)=\int_{0}^{1}\left[\frac{\epsilon^{2}}{2}\left|u^{\prime}\right|^{2}+W(u)+\frac{1}{2}\left|\left(-D^{2}\right)^{-1 / 2}(u-m)\right|^{2}\right] \mathrm{d} x,
$$

which we call the energy of $u$. The second order derivative operator

$$
-D^{2}:\left\{v \in W^{2,2}: v^{\prime}(0)=v^{\prime}(1)=0, \int_{0}^{1} v=0\right\} \rightarrow\left\{w \in L^{2}: \int_{0}^{1} w=0\right\}
$$

is an isometry. Its inverse is positive from $\left\{w \in L^{2}: \int_{0}^{1} w=0\right\}$ to itself. We denote the square root of this inverse by $\left(-D^{2}\right)^{-1 / 2}$. For every $u \in X_{m}$ we can solve

$$
-v^{\prime \prime}=u-m, \quad v^{\prime}(0)=v^{\prime}(1)=0, \quad \int_{0}^{1} v=0
$$

for $v$. This $v$ is often denoted by $\left(-D^{2}\right)^{-1}(u-m)$. Then 1.2 becomes

$$
I_{\epsilon}(u)=\int_{0}^{1}\left[\frac{\epsilon^{2}}{2}\left|u^{\prime}\right|^{2}+W(u)+\frac{1}{2}\left|v^{\prime}\right|^{2}\right] \mathrm{d} x .
$$

Let $u_{\epsilon}$ be a global minimum of $I_{\epsilon}$ in $X_{m}$, i.e.

$$
I_{\epsilon}\left(u_{\epsilon}\right)=\min _{u \in X_{m}} I_{\epsilon}(u) .
$$

The existence of $u_{\epsilon}$ is guaranteed by the usual variational argument. $u_{\epsilon}$ solves the Euler-Lagrange equation

$$
-\epsilon^{2} u^{\prime \prime}+f(u)+\left(-D^{2}\right)^{-1}(u-m)=\lambda
$$

where $f=W^{\prime}$. The constant $\lambda$, the Lagrange multiplier, is unknown. 
Defining $v_{\epsilon}=\left(-D^{2}\right)^{-1}\left(u_{\epsilon}-m\right)$ and $\lambda_{\epsilon}$ to be the Lagrange multiplier associated with $u_{\epsilon}$, we rewrite the Euler-Lagrange equation for $u_{\epsilon}, v_{\epsilon}$ and $\lambda_{\epsilon}$ as

$$
\left\{\begin{array}{l}
-\epsilon^{2} u^{\prime \prime}+f(u)+v=\lambda, \\
-v^{\prime \prime}=u-m, \\
u^{\prime}(0)=u^{\prime}(1)=v^{\prime}(0)=v^{\prime}(1)=0, \\
\int_{0}^{1} u=m, \quad \int_{0}^{1} v=0 .
\end{array}\right.
$$

Note that without the nonlocal interaction term in $(1.2)$ we have the more familiar functional

$$
K_{\epsilon}(u)=\int_{0}^{1}\left[\frac{\epsilon^{2}}{2}\left|u^{\prime}\right|^{2}+W(u)\right] \mathrm{d} x .
$$

Minimizers in $X_{m}$ of $K_{\epsilon}$ are well known. When $\epsilon$ is small, $K_{\epsilon}$ has two global minima. One of them has a transition layer, whose width is of order $\epsilon$, from -1 to 1 . The second is the reversal, i.e. the reflection with respect to the vertical line at $1 / 2$, of the first (see Carr, Gurtin and Slemrod [1]).

The goal of this paper is to prove the following three theorems for the global minima of the nonlocal problem $I_{\epsilon}$.

THEOREM 1.1 For small $\epsilon$ every global minimum $u_{\epsilon}$ is necessarily periodic, with exactly $N_{\epsilon} / 2$ periods, where $N_{\epsilon}$ is the number of transition layers of $u_{\epsilon}$.

THEOREM 1.2 For small $\epsilon, I_{\epsilon}$ has either two or four global minima. The case of two global minima is generic.

THEOREM 1.3 The period of the global minima of $I_{\epsilon}$ has the asymptotic expansion

$$
\left(\frac{96 c_{0} \epsilon}{\left(1-m^{2}\right)^{2}}\right)^{1 / 3}+O\left(\epsilon^{2 / 3}\right),
$$

where $c_{0}$ is defined in 2.6.

The proofs are rather straightforward, though some estimates in this paper look tedious. We obtain sharp lower and upper bounds for $I_{\epsilon}\left(u_{\epsilon}\right)$. The upper bound is deduced by a test function argument. The lower bound, which is harder to come by, comes after a careful study of $u_{\epsilon}$.

With these bounds we study the length scale between adjacent transition layers of $u_{\epsilon}$. A layer is characterized by a point $x$ where $u_{\epsilon}(x)$ is not close to -1 or 1 . For technical reasons we set a value $\alpha \in(-1,1)$, defined in 2.5 , and say that $x$ is an $\alpha$-point if $u_{\epsilon}(x)=\alpha$. An $\alpha$-point thus identifies a transition layer. We show that the distance between any two adjacent $\alpha$-points of $u_{\epsilon}$ is comparable to $\epsilon^{1 / 3}$.

The proof of this fact is in Sections 6 and 7. We denote intervals separated by the $\alpha$-points by $p_{i}$ and $q_{i}$. On a $p_{i}$ interval, $u_{\epsilon}$ is greater than $\alpha$, and on a $q_{i}$ interval, it is less than $\alpha$. In Proposition 6.1 we show that $p_{i}=O\left(\epsilon^{1 / 3}\right)$ and $q_{i}=O\left(\epsilon^{1 / 3}\right)$. Then in Proposition 7.1 we improve the two estimates to $p_{i} \sim \epsilon^{1 / 3}$ and $q_{i} \sim \epsilon^{1 / 3}$.

Proposition 7.1 has the implication that the distance between any two adjacent zeros of $v_{\epsilon}^{\prime}$ is also comparable to $\epsilon^{1 / 3}$. This allows us to localize $I_{\epsilon}$ to intervals separated by these zeros. After rescaling such intervals to $(0,1)$ we obtain a functional similar to $I_{\epsilon}$, but with a different parameter range. This new functional was the same as the one studied by the authors in [13]. The three theorems follow from some convexity properties of the functional $I_{\epsilon}$. 
The most important step in proving Proposition 6.1 is the establishment of a good lower bound for $I_{\epsilon}\left(u_{\epsilon}\right)$ in Section 5. This idea was used by Ni, Takagi and the second author in a series of papers (e.g. [7, 8, 9, 21]), but in different settings. There the solutions are all spiky, instead of being periodic.

The special case that $m=0$ and $W(-r)=W(r)$ was studied by Müller in [5]. He actually had a different looking functional

$$
\tilde{I}_{\epsilon}(w)=\int_{0}^{1}\left[\epsilon^{2}\left|w^{\prime \prime}\right|^{2}+\tilde{W}\left(w^{\prime}\right)+w^{2}\right] \mathrm{d} x
$$

in the admissible set $\left\{w \in W^{2,2}(0,1): w(0)=w(1)=0\right\}$. Under the assumption $W(r)=W(-r)$, it was proved in [5] that global minima of $\tilde{I}_{\epsilon}$ are periodic.

$\tilde{I}_{\epsilon}$ itself has an interpretation in the elasticity theory. Imagine $w$ as the displacement of an elastic bar under a loading device. $w^{\prime}$ is the strain field. The deformation of $w$ gives rise to some elastic energy whose density is $\epsilon^{2}\left|w^{\prime \prime}\right|^{2}+W\left(w^{\prime}\right)$. Also assume that the bar is placed on an elastic foundation. The foundation interacts with the bar and contributes to some more energy with density $w^{2}$. Adding these two terms we arrive at $\tilde{I}_{\epsilon}$, the total energy of the system. See Truskinovsky and Zanzotto [19, 20] for more details.

To see how $\tilde{I}_{\epsilon}$ is related to $I_{\epsilon}$, let $u$ be an element in $X_{m}$ and $v=\left(-D^{2}\right)^{-1}(u-m)$. Set $w=v^{\prime}$. Then $w^{\prime}=v^{\prime \prime}=m-u, w^{\prime \prime}=-u^{\prime}$, and

$$
I_{\epsilon}(u)=\frac{1}{2} \int_{0}^{1}\left[\epsilon^{2}\left|w^{\prime \prime}\right|^{2}+2 W\left(m-w^{\prime}\right)+w^{2}\right] \mathrm{d} x=\frac{1}{2} \tilde{I}_{\epsilon}(w),
$$

if $\tilde{W}(r)=2 W(m-r)$. Since both $\tilde{W}$ and $W$ have two global minima at -1 and $1, m$ must be 0 . What was proved in [5] translates to the statement that when $W(r)=W(-r)$ and $m=0$, the global minimizers of $I_{\epsilon}$ are periodic.

$W(r)=W(-r)$ may look like a technical restriction, but actually, together with $m=0$, it imposes mathematically a symmetry within each period of a minimizer $u_{\epsilon}$. If $T$ is a period, then $u_{\epsilon}(x)=-u_{\epsilon}(T-x)$ for $x \in(0, T)$. The use of this symmetry is a key ingredient in [5]. In terms of applications $m=0$ requires that each of the two monomer units make exactly half of the volume, which is not a suitable condition for general copolymers.

We will prove the three theorems without assuming $W(r)=W(-r)$ or $m=0$. Within each period, $u_{\epsilon}$ has no more symmetry. Instead $u_{\epsilon}$ is close to 1 on a portion of the period and close to -1 on another portion, generally of a different size, leaving the average of $u_{\epsilon}$ equal to $m$.

Our approach to the general case departs significantly from Müller's, when we analyze the important quantity $E(\epsilon, l)$, defined at the beginning of Section 10 . Here $l$ is the distance between two adjacent zeros of $v_{\epsilon}^{\prime}$. In the symmetric case $(W(r)=W(-r), m=0) E$ is convex with respect to $l$ in a wide range of $\epsilon$ and $l: \epsilon \leqslant C l /|\log l|$, as shown in [5]. This fact depends on a lower bound for eigenvalues of a linear problem (see Proposition 9.1 and the remark after its proof), when the symmetry condition is imposed. Without symmetry that linear problem has small eigenvalues. It turns out that the convexity of $E$ is valid if we can show that $\epsilon$ and $l$ lie in a narrower range: $C_{1} \epsilon^{1 / 3} \leqslant l \leqslant C_{2} \epsilon^{1 / 3}$. The remarks earlier after the statements of the three theorems explained how we prove this difficult estimate.

Other references on this subject include Ohnishi et al. [11], Fife and Hilhorst [3], Choksi [2], Henry [4], Ren and Wei [14]-[18], and Muratov [6]. 
When estimating quantities, we adopt $O(\ldots), o(\ldots), \sim$ convention. A term, say $v_{\epsilon}$, satisfies $v_{\epsilon}=O\left(\epsilon^{1 / 3}\right)$ if there exists a constant $C$ independent of $\epsilon$ such that $\left|v_{\epsilon}(x)\right| \leqslant C \epsilon^{1 / 3}$ for all $x \in(0,1)$. A term, say $v_{\epsilon}$, satisfies $v_{\epsilon}=o\left(\epsilon^{1 / 3} \log \epsilon\right)$ if there exists a function $C(\epsilon), C(\epsilon) \searrow 0$ as $\epsilon \searrow 0$, such that $\left|v_{\epsilon}(x)\right| \leqslant C(\epsilon)\left|\epsilon^{1 / 3} \log \epsilon\right|$ for all $x . O(\ldots)$ and $o(\ldots)$ also appear in inequalities. For instance, a term, say $u_{\epsilon}$, satisfies $u_{\epsilon} \leqslant 1+O\left(\epsilon^{1 / 3}\right)$ if there exists $C>0$ such that $u_{\epsilon}(x)-1 \leqslant$ $C \epsilon^{1 / 3}$ for all $x$. $\sim$ indicates a comparability relation between two quantities. A term, say $p_{i}$, satisfies $p_{i} \sim \epsilon^{1 / 3}$ if there exist constants $C_{1}$ and $C_{2}$ such that $C_{1} \epsilon^{1 / 3} \leqslant p_{i} \leqslant C_{2} \epsilon^{1 / 3}$ for all $i$.

We require that all estimating quantities, like $C, C_{1}, C_{2}$, or $C(\cdot)$, depend on $m$ and the overall shape of $W$ only. Therefore all estimates involving $O, o$ or $\sim$ in this paper are uniform with respect to any variable/parameter that may appear, like $x$ in $v_{\epsilon}(x)$ and $i$ in $p_{i}$.

\section{The local energy functional $K_{\epsilon}$}

The function $W$ in the definition of $I_{\epsilon}$ is a balanced double well. More precisely:

1. $W:(-\infty, \infty) \rightarrow[0, \infty)$ is $C^{5}$.

2. $W(r)=0$ at $r=-1$ and $r=1$, and $W(r)>0$ at any other $r$.

3. There exist $a$ and $b, a>-1, a<b, b<1$ such that $W^{\prime \prime}(r)>0$ on $(-\infty, a) \cup(b, \infty)$ and $W^{\prime \prime}(r)<0$ on $(a, b)$.

4. $W^{\prime \prime}$ is bounded.

5. $W^{\prime}$ grows linearly, i.e. there exist $C_{1}$ and $C_{2}$ such that $C_{1}|r| \leqslant\left|W^{\prime}(r)\right| \leqslant C_{2}|r|$ when $r$ is large.

We have made these conditions consistent with the ones in the reference papers, like [1,5]. The derivative of $W$ is always denoted by $f$, and the local maximum of $W$ between -1 and 1 by $\omega$.

Next we list some well-known properties of the equation

$$
-U^{\prime \prime}+f(U)=0 .
$$

It has the first integral

$$
-\left|U^{\prime}\right|^{2}+2 W(U)=2 \gamma .
$$

This first integral gives us a phase portrait of trajectories in the $U$ vs. $U^{\prime}$ plane. The two equilibria $(-1,0),(1,0)$ correspond to the two global minima of $W$ at -1 and 1 . The third equilibrium $(\omega, 0)$, $\omega \in(-1,1)$, comes from the local maximum $\omega$ of $W$. There are two heteroclinic orbits connecting $(-1,0)$ to $(1,0)$. They bound a family of periodic trajectories that in turn enclose $(\omega, 0)$. The remaining trajectories are unbounded.

One heteroclinic solution is denoted by $H$ which solves

$$
-H^{\prime \prime}+f(H)=0, \quad H(0)=\alpha, \quad H( \pm \infty)= \pm 1 .
$$

The constant $\alpha$ is a number between -1 and 1 defined later in 2.77 to identify transition layers. $H$ has the first integral

$$
-\left|H^{\prime}\right|^{2}+2 W(H)=0 .
$$

Lemma 2.1 1. There exists $C>0$ such that as $t \rightarrow \pm \infty, H(t)= \pm 1+O\left(\mathrm{e}^{-C|t|}\right), H^{\prime}( \pm t)=$ $O\left(e^{-C t}\right)$, and $H^{\prime \prime}( \pm t)=O\left(\mathrm{e}^{-C t}\right)$. 
2. Let $G_{s}, s>0$, be the increasing solution of $-G_{s}^{\prime \prime}+f\left(G_{s}\right)=0$ with $G_{s}(0)=\alpha$ and $G^{\prime}(s)$ $=0$. Then $\left\|G_{s}-H\right\|_{L^{\infty}(0, s)}=O\left(\mathrm{e}^{-v s}\right)$ for a constant $v>0$. If $G_{s}$ is the decreasing solution of the same equation and boundary conditions, then $\left\|G_{s}-H(-\cdot)\right\|_{L^{\infty}(0, s)}=O\left(\mathrm{e}^{-v s}\right)$.

Proof. 1. From 2.4 we obtain

$$
t=\int_{0}^{t} \mathrm{~d} \tau=\int_{0}^{t} \frac{\mathrm{d} H(\tau)}{H^{\prime}(\tau)}=\int_{\alpha}^{H(t)} \frac{\mathrm{d} H}{\sqrt{2 W(H)}} \sim-\log (1-H(t)) .
$$

The convergence rates at $\infty$ then follow. The case of $t \rightarrow-\infty$ is similar.

2. The constant $\gamma$ in 2.2 is $W\left(G_{s}(s)\right)$ when $U=G_{s}$. The estimate in this part follows by comparing the time variable

$$
t=\int_{0}^{t} \mathrm{~d} \tau=\int_{0}^{G_{s}(t)} \frac{\mathrm{d} G_{s}}{\sqrt{2 W\left(G_{s}\right)-2 W\left(G_{s}(s)\right)}}
$$

of $G_{s}$ with that of $H$ in part 1 .

LEMma 2.2 1. Let $G$ be a bounded solution of $-\Psi^{\prime \prime}+f^{\prime}(H) \Psi=0$ on $(a, \infty),(-\infty, a)$, or $(-\infty, \infty)$, where $H$ is the heteroclinic solution defined in $(2.3)$. Then there exists a constant $c$ such that $\Psi=c H^{\prime}$ and $H^{\prime} \in W^{1,2}(-\infty, \infty)$.

2. There exists a constant $\iota>0$ such that for very $\Phi \in W^{1,2}(-\infty, \infty)$ with

$$
\int_{-\infty}^{\infty} \Phi H^{\prime} \mathrm{d} t=0, \quad \int_{-\infty}^{\infty}\left[\left|\Phi^{\prime}\right|^{2}+f^{\prime}(H) \Phi^{2}\right] \mathrm{d} t \geqslant \iota \int_{-\infty}^{\infty} \Phi^{2} \mathrm{~d} t .
$$

Proof. 1. $H^{\prime}$ is obviously a solution of the linear equation. It is bounded and positive. Another linearly independent solution is $R(t)=H^{\prime}(t) \int_{0}^{t} \mathrm{~d} s /\left(H^{\prime}(s)\right)^{2}$. Then there exist $c$ and $c^{*}$ such that $\Psi=c H^{\prime}+c^{*} R$. However $R( \pm \infty)= \pm \infty$, while $\Psi$ is bounded. So $c^{*}=0$.

To see that $H^{\prime} \in W^{1,2}(-\infty, \infty)$ we return to the first integral 2.4 , the equation 2.3 , and the phase portrait, to compute

$$
\int_{-\infty}^{\infty}\left(H^{\prime}(t)\right)^{2} \mathrm{~d} t=\int_{-1}^{1} \sqrt{2 W(H)} \mathrm{d} H, \quad \int_{-\infty}^{\infty}\left(H^{\prime \prime}(t)\right)^{2} \mathrm{~d} t=\int_{-1}^{1} \frac{(f(H))^{2}}{\sqrt{2 W(H)}} \mathrm{d} H .
$$

Both integrals on the right sides are convergent.

2. $H$ is a global minimum of $\int_{-\infty}^{\infty}\left[\frac{1}{2}\left|G^{\prime}\right|^{2}+W(G)\right] \mathrm{d} t$ in $\left\{G \in W_{1 \mathrm{loc}}^{1,2}(-\infty, \infty): G( \pm \infty)= \pm 1\right\}$. 0 is the principal eigenvalue of the second variation at $H$, corresponding to an eigenfunction $H^{\prime}$. The next eigenvalue gives rise to $\iota$.

Let $\alpha \in(-1,1)$ be the number so that

$$
\frac{\int_{\alpha}^{1} \sqrt{W(s)} \mathrm{d} s}{1+m}=\frac{\int_{-1}^{\alpha} \sqrt{W(s)} \mathrm{d} s}{1-m} .
$$

Also define

$$
c_{-1}=\sqrt{2} \int_{-1}^{\alpha} \sqrt{W(s)} \mathrm{d} s, \quad c_{1}=\sqrt{2} \int_{\alpha}^{1} \sqrt{W(s)} \mathrm{d} s, \quad c_{0}=c_{-1}+c_{1} .
$$


(2.5) implies that

$$
\frac{c_{1}}{1+m}=\frac{c_{-1}}{1-m} .
$$

The number $\alpha$ will be used to identify transition layers. If $u_{\epsilon}$ is a global minimum of $I_{\epsilon}$ in $X_{m}$, we say $x \in(0,1)$ is an $\alpha$-point of $u_{\epsilon}$ if $u_{\epsilon}(x)=\alpha$. Of course any number in $(-1,1)$ can be used to identify transition layers of $u_{\epsilon}$. The reason why we choose this particular value will come out in Section 6.

Finally we consider the functional $K_{\epsilon}$ in (1.6) on various admissible sets. Let

$$
\begin{aligned}
k(\epsilon) & =\min \left\{K_{\epsilon}(u): u \in X_{m}\right\}, \\
k_{-1}(\epsilon) & =\min \left\{K_{\epsilon}(u): u \in W^{1,2}(0,1), u(0)=u(1)=\alpha, u \leqslant \alpha\right\}, \\
k_{1}(\epsilon) & =\min \left\{K_{\epsilon}(u): u \in W^{1,2}(0,1), u(0)=u(1)=\alpha, u \geqslant \alpha\right\}, \\
k_{-1}^{h}(\epsilon) & =\min \left\{K_{\epsilon}(u): u \in W^{1,2}(0,1), u(0)=\alpha, u \leqslant \alpha\right\}, \\
k_{1}^{h}(\epsilon) & =\min \left\{K_{\epsilon}(u): u \in W^{1,2}(0,1), u(0)=\alpha, u \geqslant \alpha\right\} .
\end{aligned}
$$

LEMmA 2.3 There exists $\mu>0$ for the following statements.

1. $k(\epsilon)=c_{0} \epsilon+O\left(\epsilon^{-\mu / \epsilon}\right)$.

2. $k_{-1}(\epsilon)=2 c_{-1} \epsilon+O\left(e^{-\mu / \epsilon}\right)$.

3. $k_{1}(\epsilon)=2 c_{1} \epsilon+O\left(e^{-\mu / \epsilon}\right)$.

4. $k_{-1}^{h}(\epsilon)=c_{-1} \epsilon+O\left(e^{-\mu / \epsilon}\right)$.

5. $k_{1}^{h}(\epsilon)=c_{1} \epsilon+O\left(e^{-\mu / \epsilon}\right)$.

Proof. Part 1 was proved in [1, Theorem 8.1]. The proofs of 2-5 are standard and we only show a sketch for 5 .

Recall $H$ in 2.3 . Use $H(x / \epsilon) \geqslant \alpha$ on $(0,1)$ as a test function to compute $K_{\epsilon}(H(\cdot / \epsilon))$. Because of 2.3 , we find

$$
K_{\epsilon}(H(\cdot / \epsilon))=\sqrt{2} \int_{0}^{1} \sqrt{W(H)} H^{\prime}(x / \epsilon) \mathrm{d} x=\epsilon \sqrt{2} \int_{\alpha}^{H(1 / \epsilon)} \sqrt{W(H(t))} \mathrm{d} t .
$$

Due to the exponential convergence rate of $H(t) \rightarrow 1$ as $t \rightarrow \infty$ (Lemma 2.1.),

$$
k_{1}^{h}(\epsilon) \leqslant \epsilon c_{1}+O\left(\epsilon^{-C / \epsilon}\right) \text {. }
$$

Now we show that the inequality (2.9) is indeed an equality. Let $w_{\epsilon}$ be a global minimum of $K_{\epsilon}$ in the admissible set $\left\{u \in W^{1,2}(0,1): u(0)=\alpha, u \geqslant \alpha\right\}$, whose existence is guaranteed by the theory of obstacle problems. Then $w_{\epsilon}$ satisfies the variational inequality

$$
\int_{0}^{1}\left[\epsilon^{2} w_{\epsilon}^{\prime}\left(\phi^{\prime}-w_{\epsilon}^{\prime}\right)+f\left(w_{\epsilon}\right)\left(\phi-w_{\epsilon}\right)\right] \mathrm{d} x \geqslant 0
$$

for every $\phi$ in the same admissible set.

The theory of variational inequalities asserts that $w_{\epsilon} \in W^{2,2}(0,1)$. Let $S=\{x \in(0,1)$ : $\left.w_{\epsilon}(x)=\alpha\right\}, U=(0,1) \backslash S$. Then $U$ is open and $S$ relatively closed in $(0,1)$. We show that $S=\emptyset$. Let $\bar{x} \in S$. Then $w_{\epsilon}(\bar{x})=\alpha$ and $w_{\epsilon}^{\prime}(\bar{x})=0$. It follows from 2.10p that

$$
-\epsilon^{2} w_{\epsilon}^{\prime \prime}+f\left(w_{\epsilon}\right)=0
$$

on $U$. If we multiply the equation by $w_{\epsilon}^{\prime}$, then since $w_{\epsilon}^{\prime}=0$ on $S$, on the whole $(0,1)$ there is a first 
integral

$$
-\epsilon^{2}\left|w_{\epsilon}^{\prime}\right|^{2}+2 W\left(w_{\epsilon}\right)=-\epsilon^{2}\left|w_{\epsilon}^{\prime}(\bar{x})\right|^{2}+2 W\left(w_{\epsilon}(\bar{x})\right)=2 W(\alpha) .
$$

This implies that $W\left(w_{\epsilon}\right) \geqslant W(\alpha)$. Then $K_{\epsilon}\left(w_{\epsilon}\right) \geqslant W(\alpha)>0$, which is inconsistent with 2.9p for small $\epsilon$. This proves that no such $\bar{x}$ exists and $S=\emptyset$. So $w_{\epsilon}$ solves (2.11) on $(0,1)$.

At $x=1,2.10$ allows two possibilities:

A: $w_{\epsilon}(1)>\alpha$ and $w_{\epsilon}^{\prime}(1)=0$, or

B: $w_{\epsilon}(1)=\alpha$.

We first consider case A. Set $x=\epsilon t, U(t)=w_{\epsilon}(\epsilon t)$. We suppress the dependence of $U$ on $\epsilon$ to keep notations simple. $U$ satisfies (2.2). The constant $\gamma$ there can be evaluated at $t=1 / \epsilon$ where $U^{\prime}(1 / \epsilon)=0$. So $\gamma$ by $W(U(1 / \epsilon))$.

As $\epsilon \searrow 0$, we have $U^{\prime}(0) \nearrow H^{\prime}(0), \gamma \searrow 0$ and the trajectory of $U$, which is a periodic orbit inside the two heteroclinic orbits, approaches that of $H$. It also follows that $U(1 / \epsilon)$ tends to 1 from the left. Without ambiguity, for small $\epsilon$ denote this $U(1 / \epsilon)=W^{-1}(\gamma)$.

Now we view $\gamma$, instead of $\epsilon$, as the controling parameter. (2.2) implies that the duration is

$$
\frac{1}{\epsilon}=\int_{0}^{1 / \epsilon} \mathrm{d} t=\int_{\alpha}^{W^{-1}(\gamma)} \frac{\mathrm{d} U}{\sqrt{2(W(U)-\gamma)}} \sim \log \gamma
$$

and the local energy satisfies the estimate

$$
\begin{aligned}
\epsilon^{-1} K_{\epsilon}\left(w_{\epsilon}\right)-c_{1} & =\int_{0}^{1 / \epsilon}\left[\frac{\left|U^{\prime}\right|^{2}}{2}+W(U)\right] \mathrm{d} t-c_{1}=\int_{\alpha}^{W^{-1}(\gamma)} \frac{2 W(U)-\gamma}{\sqrt{2(W(U)-\gamma)}} \mathrm{d} U-c_{1} \\
& \sim \gamma \log \gamma
\end{aligned}
$$

as $\gamma \searrow 0$. This yields the estimate in 5. of this lemma.

Finally we rule out case B. If we again set $U=w_{\epsilon}(\epsilon t)$, then in the phase portrait this solution corresponds to a part of a periodic trajectory as well. However at $t=1 / \epsilon,\left(U(1 / \epsilon), U^{\prime}(1 / \epsilon)\right)$ is the mirror image of $\left(U(0), U^{\prime}(0)\right)$ about the horizontal axis. After a similar argument of phase plane analysis, we find $K_{\epsilon}\left(w_{\epsilon}\right)=2 c_{1} \epsilon+O\left(e^{-\mu / \epsilon}\right)$, contradicting 2.9.

The constants $\mu$ in Lemma 2.3 and $v$ in Lemma 2.1 are henceforth fixed. They depend on $W$ and $m$ only.

Lemma 2.4 Let $w_{\epsilon}$ be a global minimum of $K_{\epsilon}$ in $X_{m}$. Define

$$
w_{1}(x)=\left\{\begin{array}{ll}
-1, & x \in\left(0, \frac{1-m}{2}\right), \\
1, & x \in\left(\frac{1-m}{2}, 1\right),
\end{array} \quad w_{2}(x)= \begin{cases}1, & x \in\left(0, \frac{1+m}{2}\right), \\
-1, & x \in\left(\frac{1+m}{2}, 1\right) .\end{cases}\right.
$$

Then either $\int_{0}^{1}\left|w_{\epsilon}-w_{1}\right| \mathrm{d} y=O(\epsilon \log \epsilon)$, or $\int_{0}^{1}\left|w_{\epsilon}-w_{2}\right| \mathrm{d} y=O(\epsilon \log \epsilon)$. For small $\epsilon, w_{\epsilon}$ is increasing in the first case and decreasing in the second case.

Proof. See Theorems 3.1 and 9.1 of [1].

\section{An upper bound of $I_{\epsilon}\left(u_{\epsilon}\right)$}

Let us agree on the notation $\operatorname{Ave}(w)$ for the mean of $w$, i.e. if $w$ is defined on $(a, b)$ then

$$
\operatorname{Ave}(w)=\frac{\int_{a}^{b} w(x) \mathrm{d} x}{b-a} .
$$


Lemma 3.1 1. For every positive integer $N$,

$$
I_{\epsilon}\left(u_{\epsilon}\right) \leqslant c_{0} \epsilon N+\frac{\left(1-m^{2}\right)^{2}}{24 N^{2}}+O\left(-\frac{\epsilon}{N} \log (\epsilon N)+\mathrm{e}^{-\mu /(\epsilon N)}\right) .
$$

2. If $N$ is taken to be the integer closest to $\left(\frac{\left(1-m^{2}\right)^{2}}{12 \epsilon c_{0}}\right)^{1 / 3}$, then

$$
I_{\epsilon}\left(u_{\epsilon}\right) \leqslant c_{0}^{2 / 3}\left(1-m^{2}\right)^{2 / 3}\left(\frac{9}{32}\right)^{1 / 3} \epsilon^{2 / 3}+O\left(\epsilon^{4 / 3} \log \epsilon\right) .
$$

Proof. Let $N$ be a positive integer and $(0,1)$ be equally divided by $N$. Set $l=1 / N$. Minimize over $u \in W^{1,2}(0, l)$, subject to $\operatorname{Ave}(u)=m$, the quantity

$$
\int_{0}^{l}\left[\frac{\epsilon^{2}}{2}\left|u^{\prime}\right|^{2}+W(u)\right] \mathrm{d} x
$$

to find $u_{0, \epsilon}$. By rescaling $x=l z$, we see that $\mathcal{U}_{0, \epsilon}(z)=u_{0, \epsilon}(l z)$ minimizes $K_{\epsilon / l}$ in $X_{m}$ and

$$
\begin{aligned}
\int_{0}^{l}\left[\frac{\epsilon^{2}}{2}\left|u_{0, \epsilon}^{\prime}\right|^{2}+W\left(u_{0, \epsilon}\right)\right] \mathrm{d} x & =l \int_{0}^{1}\left[\frac{\epsilon^{2}}{2 l^{2}}\left|\mathcal{U}_{0, \epsilon}^{\prime}\right|^{2}+W\left(\mathcal{U}_{0, \epsilon}\right)\right] \mathrm{d} z \\
& =l K_{\epsilon / l}\left(\mathcal{U}_{0, \epsilon}\right)=l k(\epsilon / l) .
\end{aligned}
$$

Extending $u_{0, \epsilon}$ to $(0,1)$ by anti-symmetric reflection and using it as a test function for an upper bound of $I_{\epsilon}\left(u_{\epsilon}\right)$, we find

$$
\int_{0}^{1}\left[\frac{\epsilon^{2}}{2}\left|u_{0, \epsilon}^{\prime}\right|^{2}+W\left(u_{0, \epsilon}\right)\right] \mathrm{d} x=N l k\left(\frac{\epsilon}{l}\right)=k(\epsilon N)=c_{0} \epsilon N+O\left(\mathrm{e}^{-\mu /(\epsilon N)}\right),
$$

where the last equation comes from Lemma 2.3

To estimate the nonlocal part of $I_{\epsilon}\left(u_{0, \epsilon}\right)$, let $v_{0, \epsilon}$ be the solution of $-v^{\prime \prime}=u_{0, \epsilon}-m, v^{\prime}(0)=$ $v^{\prime}(l)=0, \operatorname{Ave}(v)=0$. Through anti-symmetric reflection $v_{0, \epsilon}$ is extended to $(0,1)$ and $v_{0, \epsilon}=$ $\left(-D^{2}\right)^{-1}\left(u_{0, \epsilon}-m\right)$.

Estimate $v_{0, \epsilon}$ by comparing it with $v_{0}$ which solves $-v^{\prime \prime}=u_{0}-m, v^{\prime}(0)=v^{\prime}(l)=0$, Ave $(v)$ $=0$. Here $u_{0}$ is a step function with one jump from -1 to 1 , satisfying Ave $\left(u_{0}\right)=m$. Scale $(0, l)$ to $(0,1)$. Let $\mathcal{U}_{0}(z)=u_{0}(l z)$, i.e.

$$
\mathcal{U}_{0}(z)= \begin{cases}-1, & z \leqslant(1-m) / 2 \\ 1, & z>(1-m) / 2\end{cases}
$$

Let $\mathcal{V}_{0}(z)=l^{-2} v_{0}(l z)$. Then $\mathcal{V}_{0}=\left(-D^{2}\right)^{-1}\left(\mathcal{U}_{0}-m\right)$.

We record the expression for $\mathcal{V}_{0}$ for later purposes:

$$
\mathcal{V}_{0}= \begin{cases}\frac{1+m}{2}\left[z^{2}-\left(\frac{1-m}{2}\right)^{2}\right]-\frac{\left(1-m^{2}\right) m}{6}, & z \in\left[0, \frac{1-m}{2}\right], \\ -\frac{1-m}{2}\left[(1-z)^{2}-\left(\frac{1+m}{2}\right)^{2}\right]-\frac{\left(1-m^{2}\right) m}{6}, & z \in\left[\frac{1-m}{2}, 1\right] .\end{cases}
$$


Recall $\mathcal{U}_{0, \epsilon}(z)=u_{0, \epsilon}(l z)$. Define $\mathcal{V}_{0, \epsilon}(z)=l^{-2} v_{0, \epsilon}(l z)$. It is clear that $\mathcal{V}_{0, \epsilon}=\left(-D^{2}\right)^{-1}\left(\mathcal{U}_{0, \epsilon}-m\right)$. Therefore $\left\|v_{0, \epsilon}\right\|_{\infty}=l^{2}\left\|\mathcal{V}_{0, \epsilon}\right\|_{\infty}=O\left(l^{2}\right)$ and $\left\|v_{0}\right\|_{\infty}=l^{2}\left\|\mathcal{V}_{0}\right\|_{\infty}=O\left(l^{2}\right)$.

Apply Lemma 2.4 to $\mathcal{U}_{0, \epsilon}$, a minimum of $K_{\epsilon / l}$, to obtain

$$
\int_{0}^{1}\left|\mathcal{U}_{0, \epsilon}-\mathcal{U}_{0}\right| \mathrm{d} z=O\left(\frac{\epsilon}{l} \log \left(\frac{\epsilon}{l}\right)\right)
$$

which yields

$$
\int_{0}^{l}\left|u_{0, \epsilon}-u_{0}\right| \mathrm{d} x=l \int_{0}^{1}\left|\mathcal{U}_{0, \epsilon}-\mathcal{U}_{0}\right| \mathrm{d} z=l O\left(\frac{\epsilon}{l} \log \left(\frac{\epsilon}{l}\right)\right)=O\left(\epsilon \log \left(\frac{\epsilon}{l}\right)\right) .
$$

Then by multiplying the equation $-D^{2} w=u_{0, \epsilon}-u_{0}$ that $v_{0, \epsilon}-v_{0}$ satisfies by $v_{0, \epsilon}+v_{0}$ and integrating by parts, we find

$$
\begin{aligned}
\int_{0}^{l}\left(\left|v_{0, \epsilon}^{\prime}\right|^{2}-\left|v_{0}^{\prime}\right|^{2}\right) \mathrm{d} x & =\int_{0}^{l}\left(v_{0, \epsilon}^{\prime}-v_{0}^{\prime}\right)\left(v_{0, \epsilon}^{\prime}+v_{0}^{\prime}\right) \mathrm{d} x=\int_{0}^{l}\left(u_{0, \epsilon}-u_{0}\right)\left(v_{0, \epsilon}+v_{0}\right) \mathrm{d} x \\
& =O(\epsilon \log (\epsilon / l))\left\|v_{0, \epsilon}+v_{0}\right\|_{\infty}=O\left(\epsilon l^{2} \log (\epsilon / l)\right) .
\end{aligned}
$$

On the interval $(0,1)$,

$$
\int_{0}^{1}\left(\left|v_{0, \epsilon}^{\prime}\right|^{2}-\left|v_{0}^{\prime}\right|^{2}\right) \mathrm{d} x=O(\epsilon l \log (\epsilon / l))=O\left(\frac{\epsilon}{N} \log (\epsilon N)\right)
$$

$\int_{0}^{1} \frac{1}{2}\left|v_{0}^{\prime}\right|^{2} \mathrm{~d} x$ can be evaluated (using $[3.4$, or see formulae (3.7) and (3.8) of [13]):

$$
\int_{0}^{1} \frac{1}{2}\left|v_{0}^{\prime}\right|^{2} \mathrm{~d} x=\frac{\left(1-m^{2}\right)^{2}}{24 N^{2}} \text {. }
$$

Thus the nonlocal part of $I_{\epsilon}\left(u_{0, \epsilon}\right)$ is bounded by

$$
\frac{\left(1-m^{2}\right)^{2}}{24 N^{2}}+O\left(\frac{\epsilon}{N} \log (\epsilon N)\right) .
$$

Combining this with 3.2), we obtain the first part of the lemma.

This estimate hints that the number of $\alpha$-points of $u_{\epsilon}$ is of order $\epsilon^{-1 / 3}$. When $N$ is taken to be the integer closest to $\left(\left(1-m^{2}\right)^{2} /\left(12 \epsilon c_{0}\right)\right)^{1 / 3}$, the optimal integer that minimizes the right side of Lemma 3.1, we derive assertion 2.

\section{Some implications of the upper bound}

Proposition 4.1 1. $\left\|v_{\epsilon}\right\|_{\infty}=O\left(\epsilon^{1 / 3}\right)$.

2. $\lambda_{\epsilon}=O\left(\epsilon^{1 / 3}\right)$.

3. $-1+O\left(\epsilon^{1 / 3}\right) \leqslant u_{\epsilon} \leqslant 1+O\left(\epsilon^{1 / 3}\right)$. 
Proof. Lemma 3.1 implies $\int_{0}^{1}\left|v_{\epsilon}^{\prime}\right|^{2} \leqslant C \epsilon^{2 / 3}$. And since $\int_{0}^{1} v_{\epsilon}=0$, we find $\left\|v_{\epsilon}\right\|_{\infty}=O\left(\epsilon^{1 / 3}\right)$. Also by the same lemma $\int_{0}^{1} W\left(u_{\epsilon}\right) \leqslant C \epsilon^{2 / 3}$. Integrating 1.5 ) we find

$$
\left|\lambda_{\epsilon}\right|=\left|\int_{0}^{1} f\left(u_{\epsilon}\right) \mathrm{d} x\right| \leqslant \int_{0}^{1}\left|f\left(u_{\epsilon}\right)\right| \mathrm{d} x \leqslant C \int_{0}^{1} W^{1 / 2}\left(u_{\epsilon}\right) \mathrm{d} x \leqslant C\left(\int_{0}^{1} W\left(u_{\epsilon}\right) \mathrm{d} x\right)^{1 / 2} \leqslant C \epsilon^{1 / 3} .
$$

The equation 1.5 ) yields $-\epsilon^{2} u_{\epsilon}^{\prime \prime}+f\left(u_{\epsilon}\right)=O\left(\epsilon^{1 / 3}\right)$. Let $x_{\epsilon}$ be a global maximum of $u_{\epsilon}$. Then $u_{\epsilon}^{\prime \prime}\left(x_{\epsilon}\right) \leqslant 0$, whether or not $x_{\epsilon}$ is on the boundary, since $u_{\epsilon}^{\prime}(0)=u_{\epsilon}^{\prime}(1)=0$. So $f\left(u_{\epsilon}\left(x_{\epsilon}\right)\right) \leqslant O\left(\epsilon^{1 / 3}\right)$, which implies $u_{\epsilon}\left(x_{\epsilon}\right) \leqslant 1+O\left(\epsilon^{1 / 3}\right)$. The lower bound for $u_{\epsilon}$ follows by a similar argument.

It is often necessary to inspect $u_{\epsilon}$ in a scale comparable to $\epsilon$. Let $x_{\epsilon} \in(0,1)$ be an arbitrary point. Introduce $t$ and $U_{\epsilon}$ so that $\epsilon t+x_{\epsilon}=x$ and $U_{\epsilon}(t)=u_{\epsilon}(x)$. According to Proposition 4.1,, 2 , $U_{\epsilon}$ satisfies

$$
-U_{\epsilon}^{\prime \prime}+f\left(U_{\epsilon}\right)=O\left(\epsilon^{1 / 3}\right)
$$

on the expanding interval $\left(-x_{\epsilon} / \epsilon,\left(1-x_{\epsilon}\right) / \epsilon\right)$. Since Proposition 4.1$\}$ implies $\left|U_{\epsilon}\right| \leqslant 1+O\left(\epsilon^{1 / 3}\right)$, the regularity theory of second order differential equations asserts that along any sequence $U_{\epsilon_{n}}$ of $U_{\epsilon}$ with $\epsilon_{n} \rightarrow 0$ there exists a subsequence that converges locally (at least) in $C^{1}$ to a function $G$ which satisfies

$$
-G^{\prime \prime}+f(G)=0, \quad-1 \leqslant G \leqslant 1
$$

on the whole interval $(-\infty, \infty)$, or a half-interval $(a, \infty)$ or $(-\infty, b)$.

Observing the phase portrait of this equation, we conclude that $G$ must be either

A: a heteroclinic solution, i.e. a translate or a reversed translate of $H$ defined in 2.3,

B: the constant solution -1 or the constant solution 1 ,

C: the constant solution $\omega$ (the local maximum of $W$ between -1 and 1), or

$\mathrm{D}$ : a periodic solution whose trajectory is bounded by the two heteroclinic orbits in the phase portrait.

Lemma 4.2 Cases C and D do not occur.

Proof. We prove this by contradiction. Suppose that $G$ is the unstable constant $\omega$ or a periodic solution. We will construct a function whose energy is lower than that of $u_{\epsilon}$, contradicting the fact that $u_{\epsilon}$ is a minimizer. To make notations manageable, any sequence or further subsequences of $u_{\epsilon}$ will still be denoted by $u_{\epsilon}$ instead of $u_{\epsilon_{n}}$.

Take a large number $\theta>3$, to be determined later. Always let $\theta$ be an integer multiple of the period of $G$ if $G$ is periodic. Without loss of generality we assume $\lim \sup x_{\epsilon} \leqslant 1 / 2$. Let $\xi$ be a smooth function defined on $(-\infty, \infty)$ so that $\xi(t)=0$ if $t \leqslant 0, \xi(t)=1$ if $t \geqslant 1,|\xi(t)| \leqslant 1$ for all $t$. For each $r \in(1, \theta-2)$ define

$$
U_{\epsilon, r}(t)= \begin{cases}U_{\epsilon}(t), & t \notin(0, \theta), \\ \left(U_{\epsilon}(t)+1\right)(1-\xi(t))-1, & 0 \leqslant t \leqslant r, \\ 2 \xi(t-r)-1, & r \leqslant t \leqslant r+1, \\ \left(U_{\epsilon}(t)-1\right) \xi(t-\theta+1)+1, & r+1 \leqslant t \leqslant \theta .\end{cases}
$$

We have replaced $U_{\epsilon}$ in the interval $(0, \theta)$ by a function which is -1 on $(1, r)$ and 1 on $(r+1, \theta-1)$. 
Similarly set

$$
F_{r}(t)= \begin{cases}G(t), & t \notin(0, \theta), \\ (G(t)+1)(1-\xi(t))-1, & 0 \leqslant t \leqslant r, \\ 2 \xi(t-r)-1, & r \leqslant t \leqslant r+1, \\ (G(t)-1) \xi(t-\theta+1)+1, & r+1 \leqslant t \leqslant \theta .\end{cases}
$$

Since $U_{\epsilon} \rightarrow G$ in $C^{1}[0, \theta], U_{\epsilon, r} \rightarrow F_{r}$ in $C^{1}[0, \theta]$. We need to choose $r$ properly to have $\int_{0}^{\theta} U_{\epsilon, t}=$ $\int_{0}^{\theta} F_{r}$, so later the function that we will construct to have lower energy will be in the admissible set $X_{m}$.

Since $\theta$ is a multiple of the period of $G$ if $G$ is periodic, we see that $\theta^{-1} \int_{0}^{\theta} G(t) \mathrm{d} t \in(-1,1)$ is independent of $\theta$. Take $\eta>0$ so small that $\theta^{-1} \int_{0}^{\theta} G(t) \mathrm{d} t \pm \eta \in(-1,1)$. First set

$$
r=r_{1}=\frac{1-\theta^{-1} \int_{0}^{\theta} G(t) \mathrm{d} t+\eta}{2} \theta .
$$

Clearly $r_{1} \in(1, \theta-2)$ when $\theta$ is large. As $\theta \rightarrow \infty$, by the definition 4.4 of $F_{r}$,

$$
\frac{1}{\theta} \int_{0}^{\theta} F_{r_{1}}(t) \mathrm{d} t \rightarrow \frac{1}{\theta} \int_{0}^{\theta} G(t) \mathrm{d} t-\eta
$$

Then set

$$
r=r_{2}=\frac{1-\theta^{-1} \int_{0}^{\theta} G(t) \mathrm{d} t-\eta}{2} \theta
$$

which is also in $(1, \theta-2)$ when $\theta$ is large. As $\theta \rightarrow \infty$,

$$
\frac{1}{\theta} \int_{0}^{\theta} F_{r_{2}}(t) \mathrm{d} t \rightarrow \frac{1}{\theta} \int_{0}^{\theta} G(t) \mathrm{d} t+\eta
$$

Therefore if we choose $\theta$ large enough then

$$
\frac{1}{\theta} \int_{0}^{\theta} F_{r_{1}}(t) \mathrm{d} t<\frac{1}{\theta} \int_{0}^{\theta} G(t) \mathrm{d} t<\frac{1}{\theta} \int_{0}^{\theta} F_{r_{2}}(t) \mathrm{d} t .
$$

After this large $\theta$ is chosen, we take $\epsilon$ so small that

$$
\frac{1}{\theta} \int_{0}^{\theta} U_{\epsilon, r_{1}}(t) \mathrm{d} t<\frac{1}{\theta} \int_{0}^{\theta} U_{\epsilon}(t) \mathrm{d} t<\frac{1}{\theta} \int_{0}^{\theta} U_{\epsilon, r_{2}}(t) \mathrm{d} t
$$

With both $\theta$ and $\epsilon$ chosen we set $r \in\left(r_{1}, r_{2}\right)$ so that $\int_{0}^{\theta} U_{\epsilon, r}(t) \mathrm{d} t=\int_{0}^{\theta} U_{\epsilon}(t) \mathrm{d} t$.

Back to the $x$-coordinate, we define $u_{\epsilon, r}(x)=U_{\epsilon, r}(t)$ which is in the admissible set $X_{m}$. We now proceed to compare the energy of $u_{\epsilon}$ and $u_{\epsilon, r}$, starting with the local part. As $\epsilon \searrow 0$,

$$
\int_{0}^{\theta}\left|U_{\epsilon, r}^{\prime}\right|^{2} \mathrm{~d} t \rightarrow \int_{0}^{\theta}\left|F_{r}^{\prime}\right|^{2} \mathrm{~d} t=\int_{0}^{1}\left|F_{r}^{\prime}\right|^{2} \mathrm{~d} t+\int_{r}^{r+1}\left|F_{r}^{\prime}\right|^{2} \mathrm{~d} t+\int_{\theta-1}^{\theta}\left|F_{r}^{\prime}\right|^{2} \mathrm{~d} t,
$$

which is bounded from above by a number independent of $\theta$ and $r$. The same is true for

$$
\int_{0}^{\theta} W\left(U_{\epsilon, r}\right) \mathrm{d} t \rightarrow \int_{0}^{\theta} W\left(F_{r}\right) \mathrm{d} t
$$


So there exists $C>0$ independent of $\theta$ and $r$ such that

$$
\int_{0}^{\theta}\left[\frac{\left|F_{r}^{\prime}\right|^{2}}{2}+W\left(F_{r}\right)\right] \mathrm{d} t \leqslant C \text {. }
$$

Then for small $\epsilon$,

$$
\int_{x_{\epsilon}}^{x_{\epsilon}+\epsilon \theta}\left[\frac{\epsilon^{2}}{2}\left|u_{\epsilon, r}^{\prime}\right|^{2}+W\left(u_{\epsilon, r}\right)\right] \mathrm{d} x=\epsilon \int_{0}^{\theta}\left[\frac{\left|U_{\epsilon, r}^{\prime}\right|^{2}}{2}+W\left(U_{\epsilon, r}\right)\right] \mathrm{d} t \leqslant 2 \epsilon C .
$$

On the other hand since $G$, periodic or unstable constant, lies strictly away from -1 and 1 , there exists $c>0$, independent of $\theta$, such that $\int_{0}^{\theta} W(G(t)) \mathrm{d} t \geqslant c \theta$. Therefore

$$
\int_{0}^{\theta}\left[\frac{\left|G^{\prime}\right|^{2}}{2}+W(G)\right] \mathrm{d} t \geqslant c \theta .
$$

Then for small $\epsilon$,

$$
\int_{x_{\epsilon}}^{x_{\epsilon}+\epsilon \theta}\left[\frac{\epsilon^{2}}{2}\left|u_{\epsilon}^{\prime}\right|^{2}+W\left(u_{\epsilon}\right)\right] \mathrm{d} x=\epsilon \int_{0}^{\theta}\left[\frac{\left|U_{\epsilon}^{\prime}\right|^{2}}{2}+W\left(U_{\epsilon}\right)\right] \mathrm{d} t \geqslant \frac{\epsilon \theta c}{2} .
$$

We see that the local energy is reduced if $\theta$ is large.

To compare the nonlocal energy we work with the $x$-coordinate. Set $v_{\epsilon, r}=\left(-D^{2}\right)^{-1}\left(u_{\epsilon, r}-m\right)$. Then $v_{\epsilon, r}^{\prime}$ and $v_{\epsilon}^{\prime}$ agree outside $\left(x_{\epsilon}, x_{\epsilon}+\epsilon \theta\right)$. Clearly $v_{\epsilon, r}^{\prime}=O(1)$ and $v_{\epsilon}^{\prime}=O(1)$ because $u_{\epsilon, r}$ and $u_{\epsilon}$ are of order $O(1)$. Since $-\left(v_{\epsilon, r}^{\prime}-v_{\epsilon}^{\prime}\right)^{\prime}=u_{\epsilon, r}-u_{\epsilon}$ on $\left(x_{\epsilon}, x_{\epsilon}+\epsilon \theta\right)$, it follows that $v_{\epsilon, r}^{\prime}-v_{\epsilon}^{\prime}=$ $O(\epsilon \theta)$ there. Then

$$
\begin{aligned}
\int_{x_{\epsilon}}^{x_{\epsilon}+\epsilon \theta}\left|v_{\epsilon, r}^{\prime}\right|^{2} \mathrm{~d} x-\int_{x_{\epsilon}}^{x_{\epsilon}+\epsilon \theta}\left|v_{\epsilon}^{\prime}\right|^{2} \mathrm{~d} x & =\int_{x_{\epsilon}}^{x_{\epsilon}+\epsilon \theta}\left(v_{\epsilon, r}^{\prime}-v_{\epsilon}^{\prime}\right)\left(v_{\epsilon, r}^{\prime}+v_{\epsilon}^{\prime}\right) \mathrm{d} x \\
& =\int_{x_{\epsilon}}^{x_{\epsilon}+\epsilon \theta} O(\epsilon \theta) \cdot O(1) \mathrm{d} x=O\left(\epsilon^{2} \theta^{2}\right) .
\end{aligned}
$$

Combining this with 4.5 and 4.6 we deduce

$$
I_{\epsilon}\left(u_{\epsilon, r}\right)-I_{\epsilon}\left(u_{\epsilon}\right) \leqslant 2 \epsilon C-\frac{\epsilon \theta c}{2}+O\left(\epsilon^{2} \theta^{2}\right) .
$$

Just as in the construction of $U_{\epsilon, r}$, we first choose $\theta$ large and then $\epsilon$ small, so $I_{\epsilon}\left(u_{\epsilon, r}\right)<I_{\epsilon}\left(u_{\epsilon}\right)$.

We first use this lemma to study $\alpha$-points of $u_{\epsilon}$. Recall from Section 2 that $x$ is an $\alpha$-point if $u_{\epsilon}(x)=\alpha$.

Proposition 4.3 When $\epsilon$ is small, $u_{\epsilon}^{\prime}\left(x_{\epsilon}\right) \neq 0$ at every $\alpha$-point $x_{\epsilon}$.

Proof. From Lemma $4.2, u_{\epsilon}\left(\epsilon t+x_{\epsilon}\right) \rightarrow G$ locally in $C^{1}$, where $G$ is heteroclinic or \pm 1 . Since $u_{\epsilon}\left(x_{\epsilon}\right)=\alpha, G(0)=\alpha$. Then $G(t)=H(t)$ or $G(t)=H(-t)(H$ is defined in 2.3.). Then $\epsilon u_{\epsilon}^{\prime}\left(x_{\epsilon}\right) \rightarrow \pm H^{\prime}(0) \neq 0$.

The proof actually says more: $u_{\epsilon}^{\prime}\left(x_{\epsilon}\right) \rightarrow \pm \infty$. Proposition 4.3 implies that the $\alpha$-points of $u_{\epsilon}$ are nondegenerate, meaning that every time the graph of $u_{\epsilon}$ touches the horizontal level $\alpha$, it crosses it. The next application of Lemma 4.2 shows that $\alpha$-points do not appear in any neighborhood of the boundary of $(0,1)$ whose size is of order $\epsilon$. 
Proposition 4.4 If $x_{\epsilon}$ is an $\alpha$-point of $u_{\epsilon}$, then

$$
\frac{\epsilon}{x_{\epsilon}}=o(1) \quad \text { and } \quad \frac{\epsilon}{1-x_{\epsilon}}=o(1) .
$$

Proof. Of course one of $\epsilon / x_{\epsilon}=o(1)$ and $\epsilon /\left(1-x_{\epsilon}\right)=o(1)$ must hold. Suppose the former is true and the latter is false. Then we can assume $\left(1-x_{\epsilon}\right) / \epsilon \rightarrow b \geqslant 0$. Let $U_{\epsilon}(t)=u_{\epsilon}\left(\epsilon t+x_{\epsilon}\right)$. Again by Lemma 4.2 $U_{\epsilon}(t)$ converges to $H(t)$ or $H(-t)$ locally in $C^{1}$. However $0=U^{\prime}\left(\left(1-x_{\epsilon}\right) / \epsilon\right) \rightarrow$ $\pm H^{\prime}(b) \neq 0$. A contradiction.

These two propositions imply that the number of $\alpha$-points is finite for each small $\epsilon$. Denote them by $x_{1}, \ldots, x_{N_{\epsilon}}$, in increasing order. We suppress the dependence of the $x_{i}$ 's on $\epsilon$ to simplify notation. Throughout the rest of the paper we assume without loss of generality that $u_{\epsilon}>0$ on $\left(0, x_{1}\right)$ and $N_{\epsilon}$ is even. We set $M_{\epsilon}=N_{\epsilon} / 2$. Let

$$
\left\{\begin{array}{llll}
p_{1}=x_{1}, & p_{2}=x_{3}-x_{2}, & \ldots, & p_{M_{\epsilon}+1}=1-x_{N_{\epsilon}}, \\
q_{1}=x_{2}-x_{1}, & q_{2}=x_{4}-x_{3}, & \ldots, & q_{M_{\epsilon}}=x_{N_{\epsilon}}-x_{N_{\epsilon}-1} .
\end{array}\right.
$$

When no confusion exists we call the interval whose length is $p_{i}$ the $p_{i}$ interval, and the interval whose length is $q_{i}$ the $q_{i}$ interval. Because of the nondegeneracy of the $x_{i}$ 's, $u_{\epsilon}>\alpha$ on every $p_{i}$ interval and $u_{\epsilon}<\alpha$ on every $q_{i}$ interval. The last interval $\left(x_{N_{\epsilon}}, 1\right)$ is $p_{M_{\epsilon}+1}$. Again the $p_{i}$ 's and $q_{i}$ 's depend on $\epsilon$. With this setting the $\alpha$-point $x_{2 i-2}$ is followed by the $p_{i}$ interval, which is followed by $x_{2 i-1}$, which is followed by the $q_{i}$ interval.

Proposition $4.5 \epsilon / p_{i}=o(1)$ and $\epsilon / q_{i}=o(1)$.

Proof. The cases of $p_{1}$ and $p_{M_{\epsilon}+1}$ are already covered by Proposition 4.4 Suppose this proposition is false. There exist adjacent $\alpha$-points $x_{\epsilon}$ and $x_{\epsilon}^{*}$ such that $\left(x_{\epsilon}^{*}-x_{\epsilon}\right) / \epsilon \rightarrow d \geqslant 0$. Again the convergence is really along a sequence $\epsilon_{n}$ of $\epsilon$, but we stay with $\epsilon$. We can assume $u_{\epsilon}>\alpha$ on $\left(x_{\epsilon}, x_{\epsilon}^{*}\right)$. Let $U_{\epsilon}(t)=u_{\epsilon}\left(\epsilon t+x_{\epsilon}\right)$.

If $d=0$, then there exists $t_{\epsilon} \in\left(0,\left(x_{\epsilon}^{*}-x_{\epsilon}\right) / \epsilon\right)$ such that $U_{\epsilon}^{\prime}\left(t_{\epsilon}\right)=0$. As $\epsilon \searrow 0$, we have $\left(x_{\epsilon}^{*}-x_{\epsilon}\right) / \epsilon \rightarrow 0$ and $t_{\epsilon} \rightarrow 0$. Also by Lemma 4.2 and the facts that $U_{\epsilon}(0)=\alpha$ and $U_{\epsilon}>\alpha$ on $\left(0,\left(x_{\epsilon}^{*}-x_{\epsilon}\right) / \epsilon\right), U_{\epsilon}(t) \rightarrow H(t)$ locally in $C^{1}$. Then $0=U_{\epsilon}^{\prime}\left(t_{\epsilon}\right) \rightarrow H^{\prime}(0) \neq 0$. A contradiction.

If $d>0$, then again $U_{\epsilon}(t) \rightarrow H(t)$. So $\alpha=U_{\epsilon}\left(\left(x_{\epsilon}^{*}-x_{\epsilon}\right) / \epsilon\right) \rightarrow H(d)$. But $H(d)=\alpha$ is impossible, since $H(0)=\alpha$ and $H$ is strictly increasing.

LEMMA 4.6 1. For $i=2, \ldots, M_{\epsilon}+1$,

$$
\begin{aligned}
\left\|u_{\epsilon}\left(\epsilon t+x_{2 i-2}\right)-H(t)\right\|_{C^{2}\left[0, p_{i} /(2 \epsilon)\right]} & =O\left(\epsilon^{1 / 3}\right)+O\left(\mathrm{e}^{-v p_{i} /(2 \epsilon)}\right), \\
\left\|u_{\epsilon}\left(\epsilon t+x_{2 i-2}\right)-H(t)\right\|_{C^{2}\left[-q_{i-1} /(2 \epsilon), 0\right]} & =O\left(\epsilon^{1 / 3}\right)+O\left(\mathrm{e}^{-v q_{i-1} /(2 \epsilon)}\right) .
\end{aligned}
$$

2. For $i=1, \ldots, M_{\epsilon}$,

$$
\begin{aligned}
\left\|u_{\epsilon}\left(\epsilon t+x_{2 i-1}\right)-H(-t)\right\|_{C^{2}\left[0, q_{i} /(2 \epsilon)\right]} & =O\left(\epsilon^{1 / 3}\right)+O\left(\mathrm{e}^{-v q_{i} /(2 \epsilon)}\right), \\
\left\|u_{\epsilon}\left(\epsilon t+x_{2 i-1}\right)-H(-t)\right\|_{C^{2}\left[-p_{i} /(2 \epsilon), 0\right]} & =O\left(\epsilon^{1 / 3}\right)+O\left(\mathrm{e}^{-v p_{i} /(2 \epsilon)}\right) .
\end{aligned}
$$

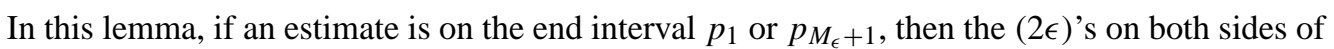
the estimate should read $\epsilon$. 
Proof. We only prove the first estimate of Lemma $4.6_{1}$, since the other three are similar. There are two different cases. When $i=1, \ldots, M_{\epsilon}, u_{\epsilon}$ is estimated on a $p_{i}$ interval with two $\alpha$-points $x_{2 i-2}$ and $x_{2 i-1}$ as the boundary. When $i=M_{\epsilon}+1, u_{\epsilon}$ is estimated on $\left(x_{2 M_{\epsilon}}, 1\right)$, an end interval. In order to study the two cases in a unified way, in this proof we extend the domain of $u_{\epsilon}$ and $v_{\epsilon}$ to $\left(0,1+p_{M_{\epsilon}+1}\right)$ by setting $u_{\epsilon}(x)=u_{\epsilon}(2-x)$ and $v_{\epsilon}(x)=v_{\epsilon}(2-x)$ for $x \in\left(1,1+p_{M_{\epsilon}+1}\right)$. then $u_{\epsilon}$ and $v_{\epsilon}$ still solve (1.5) on $\left(0,1+p_{M_{\epsilon}+1}\right)$, and $u_{\epsilon}\left(1+p_{M_{\epsilon}+1}\right)=\alpha$. Let $x=\epsilon t+x_{2 i-2}$, and $U_{\epsilon}(t)=u_{\epsilon}\left(\epsilon t+x_{2 i-2}\right)$. The proof consists of four steps.

Step 1: $\left\|U_{\epsilon}-H\right\|_{L^{\infty}\left(0, p_{i} /(2 \epsilon)\right)}=o(1)$. As $\epsilon \searrow 0$, by Proposition 4.5 $p_{i} /(2 \epsilon) \rightarrow \infty$, and by Lemma 4.2. $U_{\epsilon} \rightarrow H$ locally in $C^{1}$. If this convergence is not in $L^{\infty}\left(0, p_{i} /(2 \epsilon)\right)$, there exists $h_{\epsilon} \in\left(0, p_{i} /(2 \epsilon)\right)$ such that $\left|U_{\epsilon}\left(h_{\epsilon}\right)-H\left(h_{\epsilon}\right)\right| \not \rightarrow 0$ and $h_{\epsilon} \rightarrow \infty$. Thus $U_{\epsilon}\left(h_{\epsilon}\right)$ stays away from 1. Shift $U_{\epsilon}(t)$ to $U_{\epsilon}\left(t+h_{\epsilon}\right)$. Let $G$ be such that $U_{\epsilon}\left(t+h_{\epsilon}\right) \rightarrow G$ locally in $C^{1}$ and $-G^{\prime \prime}+f(G)=0$. Then $G$ is either 1 or heteroclinic by Lemma 4.2 . If $G=1$, then $U_{\epsilon}\left(h_{\epsilon}\right) \rightarrow 1$. A contradiction. If $G$ is heteroclinic, $G(\zeta)<\alpha$ at some $\zeta$. Then $U_{\epsilon}\left(\zeta+h_{\epsilon}\right)<\alpha$ when $\epsilon$ is small. This is impossible since for $t=\zeta+h_{\epsilon}, x=\epsilon\left(\zeta+h_{\epsilon}\right)+x_{2 i-2} \in\left(x_{2 i-2}, x_{2 i-1}\right)$ where $u_{\epsilon}>\alpha$.

Step 2: $\left\|U_{\epsilon}-H\right\|_{L^{\infty}\left(0, p_{i} /(2 \epsilon)\right)}=O\left(\epsilon^{1 / 3}\right)+O\left(\mathrm{e}^{-v p_{i} /(2 \epsilon)}\right)$. Let $G_{p_{i} /(2 \epsilon)}$ be the increasing solution of $-G^{\prime \prime}+f(G)=0$ with the boundary conditions $G_{p_{i} /(2 \epsilon)}(0)=\alpha$ and $G_{p_{i} /(2 \epsilon)}^{\prime}\left(p_{i} / \epsilon\right)=0$. Note that $G_{p_{i} /(2 \epsilon)}$ is part of a periodic trajectory in the phase plane and $G_{p_{i} /(2 \epsilon)}\left(p_{i} / \epsilon\right)=\alpha$. We first show that $\left\|U_{\epsilon}-G_{p_{i} /(2 \epsilon)}\right\|_{L^{\infty}\left(0, p_{i} / \epsilon\right)}=O\left(\epsilon^{1 / 3}\right)$.

On the contrary suppose that $\left\|U_{\epsilon}-G_{p_{i} /(2 \epsilon)}\right\|_{L^{\infty}\left(0, p_{i} / \epsilon\right)} \epsilon^{-1 / 3} \rightarrow \infty$. Let

$$
\Psi_{\epsilon}=\frac{U_{\epsilon}-G_{p_{i} /(2 \epsilon)}}{\left\|U_{\epsilon}-G_{p_{i} /(2 \epsilon)}\right\|_{L^{\infty}\left(0, p_{i} / \epsilon\right)}} .
$$

By Proposition $4.1_{1,2},-U_{\epsilon}^{\prime \prime}+f\left(U_{\epsilon}\right)=O\left(\epsilon^{1 / 3}\right)$. So $-\Psi_{\epsilon}^{\prime \prime}+f^{\prime}(\ldots) \Psi_{\epsilon}=o(1), \Psi_{\epsilon}(0)=\Psi_{\epsilon}\left(p_{i} / \epsilon\right)$ $=0$, where $f^{\prime}$ is evaluated at a number between $U_{\epsilon}$ and $G_{p_{i} /(2 \epsilon)}$, whose exact value is not important for us. We can assume that the maximum of $\left|\Psi_{\epsilon}\right|$ is achieved at $h_{\epsilon} \in\left[0, p_{i} / \epsilon\right]$, and it is a global maximum, i.e. $\Psi_{\epsilon}\left(h_{\epsilon}\right)=1$. There are three possibilities for the location of $h_{\epsilon}$ :

A: There exists $\eta>0$ such that $h_{\epsilon}<\eta$ for all $\epsilon$.

B: There exists $\eta>0$ such that $h_{\epsilon}>p_{i} / \epsilon-\eta$ for all $\epsilon$.

C: Neither of the above.

If case A occurs, by the fact that $G_{p_{i} /(2 \epsilon)} \rightarrow H$ in $L^{\infty}\left(0, p_{i} /(2 \epsilon)\right)$ as $\epsilon \rightarrow 0$, Lemma 2.1 , and Step 1, we find $\Psi_{\epsilon} \rightarrow \Psi$ locally in $C^{1}$ where $\Psi$ satisfies $-\Psi^{\prime \prime}+f^{\prime}(H) \Psi=0$ on $(0, \infty)$. Since $|\Psi| \leqslant 1$, Lemma 2.2 , asserts $\Psi=c H^{\prime}$ for some $c$. Also $\Psi(0)=\lim \Psi_{\epsilon}(0)=0$. Since $H^{\prime}(0) \neq 0$, $c=0$ and $\Psi=0$. This is clearly inconsistent with $\Psi_{\epsilon}\left(h_{\epsilon}\right)=1$ and $h_{\epsilon}<\eta$ for all small $\epsilon$.

Case B can be ruled out in the same manner. When case C occurs we assume $h_{\epsilon} \in\left(0, p_{i} / \epsilon\right)$, $h_{\epsilon} \rightarrow \infty$ and $p_{i} / \epsilon-h_{\epsilon} \rightarrow \infty$. By Step 1, or a similar assertion $\left\|U_{\epsilon}-H\left(p_{i} / \epsilon-\cdot\right)\right\|_{L^{\infty}\left(p_{i} /(2 \epsilon), p_{i} / \epsilon\right)}=$ $o(1)$, we find that in the equation for $\Psi_{\epsilon},-\Psi_{\epsilon}^{\prime \prime}\left(h_{\epsilon}\right) \geqslant 0$ (since $h_{\epsilon}$ is a maximum) and $f^{\prime}(\ldots) \Psi_{\epsilon}\left(h_{\epsilon}\right) \rightarrow f^{\prime}(1)>0$. Thus the equation cannot be satisfied at $h_{\epsilon}$ when $\epsilon$ is small. So we have proved that $\left\|U_{\epsilon}-G_{p_{i} /(2 \epsilon)}\right\|_{L^{\infty}\left(0, p_{i} / \epsilon\right)}=O\left(\epsilon^{1 / 3}\right)$. Lemma 2.1. then completes Step 2.

Step 3: $\left\|U_{\epsilon}^{\prime \prime}-H^{\prime \prime}\right\|_{L^{\infty}\left(0, p_{i} /(2 \epsilon)\right)}=O\left(\epsilon^{1 / 3}\right)+O\left(\mathrm{e}^{-v p_{i} / \epsilon}\right)$. From Steps 1, 2 and the equations 4.1 and (2.3) satisfied by $U_{\epsilon}$ and $H$ respectively,

$$
\left(U_{\epsilon}^{\prime \prime}-H^{\prime \prime}\right)=f^{\prime}(\ldots)\left(U_{\epsilon}-H\right)+O\left(\epsilon^{1 / 3}\right)=O\left(\epsilon^{1 / 3}\right)+O\left(\mathrm{e}^{-v p_{i} / \epsilon}\right) .
$$


Step 4: $\left\|U_{\epsilon}^{\prime}-H^{\prime}\right\|_{L^{\infty}\left(0, p_{i} /(2 \epsilon)\right)}=O\left(\epsilon^{1 / 3}\right)+O\left(\mathrm{e}^{-v p_{i} / \epsilon}\right)$. Let $S_{\epsilon}=U_{\epsilon}-H$. Then $S=O\left(\epsilon^{1 / 3}\right)+$ $O\left(\mathrm{e}^{-v p_{i} / \epsilon}\right)$ and $S^{\prime \prime}=O\left(\epsilon^{1 / 3}\right)+O\left(\mathrm{e}^{-v p_{i} / \epsilon}\right)$ by Steps 2 and 3 . Assume without loss of generality $h, h+1 \in\left(0, p_{i} /(2 \epsilon)\right.$.) (Otherwise consider $\left.h, h-1\right)$. Then

$$
\begin{aligned}
O\left(\epsilon^{1 / 3}\right)+O\left(\mathrm{e}^{-v p_{i} / \epsilon}\right) & =S_{\epsilon}(h+1)=S_{\epsilon}(h)+S_{\epsilon}^{\prime}(h)+\frac{1}{2} S_{\epsilon}^{\prime \prime}(\ldots) \\
& =S_{\epsilon}^{\prime}(h)+O\left(\epsilon^{1 / 3}\right)+O\left(\mathrm{e}^{-v p_{i} / \epsilon}\right)
\end{aligned}
$$

Hence $S_{\epsilon}^{\prime}(h)=O\left(\epsilon^{1 / 3}\right)+O\left(\mathrm{e}^{-v p_{i} / \epsilon}\right)$.

\section{A lower bound of $I_{\epsilon}\left(u_{\epsilon}\right)$}

A scaling in Lemma 2.3 , 3,5 yields a lower bound for the local part of $I_{\epsilon}\left(u_{\epsilon}\right)$.

LEMMA 5.1 On a $p_{i}$ or $q_{i}$ interval the local part of $I_{\epsilon}\left(u_{\epsilon}\right)$ has the lower bound

$$
\begin{aligned}
& \int_{p_{i}}\left[\frac{\epsilon^{2}}{2}\left|u_{\epsilon}^{\prime}\right|^{2}+W\left(u_{\epsilon}\right)\right] \mathrm{d} x \geqslant \begin{cases}c_{1} \epsilon+p_{i} O\left(\mathrm{e}^{-\mu p_{i} / \epsilon}\right), & i=1, M_{\epsilon}+1, \\
2 c_{1} \epsilon+p_{i} O\left(\mathrm{e}^{-\mu p_{i} / \epsilon}\right), & i \neq 1, M_{\epsilon}+1,\end{cases} \\
& \int_{q_{i}}\left[\frac{\epsilon^{2}}{2}\left|u_{\epsilon}^{\prime}\right|^{2}+W\left(u_{\epsilon}\right)\right] \mathrm{d} x \geqslant 2 c_{-1} \epsilon+q_{i} O\left(\mathrm{e}^{-\mu q_{i} / \epsilon}\right) .
\end{aligned}
$$

More difficult to find are the lower bounds for the nonlocal part of $I_{\epsilon}\left(u_{\epsilon}\right)$.

LEMMA 5.2 On a $p_{i}$ or $q_{i}$ interval the nonlocal part of $I_{\epsilon}\left(u_{\epsilon}\right)$ has the lower bound

$$
\begin{aligned}
\frac{1}{2} \int_{p_{i}}\left|v_{\epsilon}^{\prime}\right|^{2} \geqslant & \frac{(1-m)^{2}}{6} p_{i}^{3}+p_{i}^{3} O\left(\epsilon^{1 / 3}\right)+p_{i}^{2} O(\epsilon), \quad i=1, M_{\epsilon}+1, \\
\frac{1}{2} \int_{p_{i}}\left|v_{\epsilon}^{\prime}\right|^{2} \geqslant & \frac{(1-m)^{2}}{24} p_{i}^{3}+\frac{p_{i}}{2}\left[v_{\epsilon}^{\prime}\left(x_{2 i-2}\right)-\frac{(1-m) p_{i}}{2}+p_{i} O\left(\epsilon^{1 / 3}\right)+O(\epsilon)\right]^{2} \\
& +p_{i}^{3} O\left(\epsilon^{1 / 3}\right)+p_{i}^{2} O(\epsilon), \quad i \neq 1, M_{\epsilon}+1, \\
\frac{1}{2} \int_{q_{i}}\left|v_{\epsilon}^{\prime}\right|^{2} \geqslant & \frac{(1+m)^{2}}{24} q_{i}^{3}+\frac{q_{i}}{2}\left[v_{\epsilon}^{\prime}\left(x_{2 i-1}\right)+\frac{(1+m) q_{i}}{2}+q_{i} O\left(\epsilon^{1 / 3}\right)+O(\epsilon)\right]^{2} \\
& +q_{i}^{3} O\left(\epsilon^{1 / 3}\right)+q_{i}^{2} O(\epsilon), \quad i=1, \ldots, M_{\epsilon} .
\end{aligned}
$$

Proof. On $\left(0, x_{1}\right)$, with the help of Lemma 4.6, we have

$$
\begin{aligned}
v_{\epsilon}^{\prime}(x) & =v_{\epsilon}^{\prime}(0)-\int_{0}^{x}\left(u_{\epsilon}-m\right) \mathrm{d} y \\
& =-\int_{0}^{x}(1-m) \mathrm{d} y+\int_{0}^{x}\left[H\left(\frac{p_{1}-y}{\epsilon}\right)-u_{\epsilon}\right] \mathrm{d} y+\int_{0}^{x}\left[1-H\left(\frac{p_{1}-y}{\epsilon}\right)\right] \mathrm{d} y \\
& =-(1-m) x+p_{1} O\left(\epsilon^{1 / 3}\right)+p_{1} O\left(\mathrm{e}^{-v p_{1} / \epsilon}\right)+O(\epsilon) \\
& =-(1-m) x+p_{1} O\left(\epsilon^{1 / 3}\right)+O(\epsilon),
\end{aligned}
$$


where the reduction to the last line follows from the estimate $p_{1} O\left(\mathrm{e}^{-v p_{1} / \epsilon}\right)=\epsilon O\left(\left(p_{1} / \epsilon\right) \mathrm{e}^{-v p_{1} / \epsilon}\right)$ $=o(\epsilon)$. This leads to

$$
\frac{1}{2} \int_{p_{1}}\left|v_{\epsilon}^{\prime}\right|^{2} \mathrm{~d} x=\frac{(1-m)^{2} p_{1}^{3}}{6}+p_{1}^{3} O\left(\epsilon^{1 / 3}\right)+p_{1}^{2} O(\epsilon) .
$$

On $\left(x_{1}, x_{2}\right)$,

$$
v_{\epsilon}^{\prime}(x)=v_{\epsilon}^{\prime}\left(x_{1}\right)-\int_{x_{1}}^{x}\left(u_{\epsilon}-m\right) \mathrm{d} y=v_{\epsilon}^{\prime}\left(x_{1}\right)+(1+m)\left(x-x_{1}\right)+q_{1} O\left(\epsilon^{1 / 3}\right)+O(\epsilon),
$$

which implies

$$
\begin{aligned}
\frac{1}{2} \int_{q_{1}}\left|v_{\epsilon}^{\prime}\right|^{2} \mathrm{~d} x= & \frac{1}{2} \int_{q_{1}}\left[v_{\epsilon}^{\prime}\left(x_{1}\right)+(1+m)\left(x-x_{1}\right)+q_{1} O\left(\epsilon^{1 / 3}\right)+O(\epsilon)\right]^{2} \mathrm{~d} x \\
= & \frac{1}{2} \int_{q_{1}}\left[v_{\epsilon}^{\prime}\left(x_{1}\right)+(1+m)\left(x-x_{1}\right)\right]^{2} \mathrm{~d} x \\
& +v_{\epsilon}^{\prime}\left(x_{1}\right)\left[q_{1}^{2} O\left(\epsilon^{1 / 3}\right)+q_{1} O(\epsilon)\right]+q_{1}^{3} O\left(\epsilon^{1 / 3}\right)+q_{1}^{2} O(\epsilon) \\
= & \left.\frac{\left[v_{\epsilon}^{\prime}\left(x_{1}\right)+(1+m)\left(x-x_{1}\right)\right]^{3}}{6(1+m)}\right|_{x=x_{1}} ^{x=x_{2}} \\
& +v_{\epsilon}^{\prime}\left(x_{1}\right)\left[q_{1}^{2} O\left(\epsilon^{1 / 3}\right)+q_{1} O(\epsilon)\right]+q_{1}^{3} O\left(\epsilon^{1 / 3}\right)+q_{1}^{2} O(\epsilon) \\
= & \frac{1}{6(1+m)}\left[2\left(\frac{(1+m) q_{1}}{2}\right)^{3}+3(1+m) q_{1}\left(v_{\epsilon}^{\prime}\left(x_{1}\right)+\frac{(1+m) q_{1}}{2}\right)^{2}\right] \\
& +v_{\epsilon}^{\prime}\left(x_{1}\right)\left[q_{1}^{2} O\left(\epsilon^{1 / 3}\right)+q_{1} O(\epsilon)\right]+q_{1}^{3} O\left(\epsilon^{1 / 3}\right)+q_{1}^{2} O(\epsilon) \\
= & \frac{(1+m)^{2} q_{1}^{3}}{24}+\frac{q_{1}}{2}\left[v_{\epsilon}^{\prime}\left(x_{1}\right)+\frac{(1+m) q_{1}}{2}+q_{1} O\left(\epsilon^{1 / 3}\right)+O(\epsilon)\right]^{2} \\
& +q_{1}^{3} O\left(\epsilon^{1 / 3}\right)+q_{1}^{2} O(\epsilon) .
\end{aligned}
$$

We continue this argument until we reach the $q_{M_{\epsilon}}$ interval $\left(x_{N_{\epsilon}-1}, x_{N_{\epsilon}}\right)$. Finally, on $\left(x_{N_{\epsilon}}, 1\right)$ we use an estimate similar to the one on $\left(0, x_{1}\right)$, i.e. write

$$
v_{\epsilon}^{\prime}(x)=v_{\epsilon}^{\prime}(1)-\int_{1}^{x}\left(u_{\epsilon}-m\right) \mathrm{d} y=(1-m)(1-x)+p_{M_{\epsilon}+1} O\left(\epsilon^{1 / 3}\right)+O(\epsilon),
$$

to derive

$$
\frac{1}{2} \int_{p_{M_{\epsilon}+1}}\left|v_{\epsilon}^{\prime}\right|^{2} \mathrm{~d} x=\frac{(1-m)^{2} p_{M_{\epsilon}+1}^{3}}{6}+p_{M_{\epsilon}+1}^{3} O\left(\epsilon^{1 / 3}\right)+p_{M_{\epsilon}+1}^{2} O(\epsilon) .
$$

Two remarks are in order. First, the two square terms in the lemma involving $v_{\epsilon}^{\prime}\left(x_{2 i-1,2}\right)$ will be only used once, though critically, in the proof of Proposition 7.2. In the other applications they will simply be dropped.

Second, we have presented this lemma arguing first with $\left(0, x_{1}\right)$ and then proceeding to the right. As a consequence $v_{\epsilon}^{\prime}\left(x_{N_{\epsilon}}\right)$ does not appear in the estimates. Naturally, there is another version of the 
lemma where we start with $\left(x_{N_{\epsilon}}, 1\right)$ and proceed backwards. Then the second and third inequalities become

$$
\begin{aligned}
\frac{1}{2} \int_{p_{i}}\left|v_{\epsilon}^{\prime}\right|^{2} \geqslant & \frac{(1-m)^{2}}{24} p_{i}^{3}+\frac{p_{i}}{2}\left[v_{\epsilon}^{\prime}\left(x_{2 i+1}\right)+\frac{(1-m) p_{i}}{2}+p_{i} O\left(\epsilon^{1 / 3}\right)+O(\epsilon)\right]^{2} \\
& +p_{i}^{3} O\left(\epsilon^{1 / 3}\right)+p_{i}^{2} O(\epsilon), \quad i \neq 1, M_{\epsilon}+1, \\
\frac{1}{2} \int_{q_{i}}\left|v_{\epsilon}^{\prime}\right|^{2} \geqslant & \frac{(1+m)^{2}}{24} q_{i}^{3}+\frac{q_{i}}{2}\left[v_{\epsilon}^{\prime}\left(x_{2 i}\right)-\frac{(1+m) q_{i}}{2}+q_{i} O\left(\epsilon^{1 / 3}\right)+O(\epsilon)\right]^{2} \\
& +q_{i}^{3} O\left(\epsilon^{1 / 3}\right)+q_{i}^{2} O(\epsilon) .
\end{aligned}
$$

In this version $v_{\epsilon}^{\prime}\left(x_{1}\right)$ does not appear.

Lemma 5.2 yields a very rough upper bound for $p_{i}$ and $q_{i}$.

Proposition $5.3 p_{i}=O\left(\epsilon^{2 / 9}\right)$ and $q_{i}=O\left(\epsilon^{2 / 9}\right)$.

Proof. Let us consider the case of $p_{i}, i \neq 1, M_{\epsilon}+1$. The other two cases can be handled similarly. According to Lemma 5.2 .

$$
I_{\epsilon}\left(u_{\epsilon}\right) \geqslant \frac{(1-m)^{2}}{24} p_{i}^{3}+p_{i}^{3} O\left(\epsilon^{1 / 3}\right)+p_{i}^{2} O(\epsilon) .
$$

Because of Proposition 4.5 , the last two terms on the right side can be written as $p_{i}^{3} o(1)$, which is small compared to the first term on the right side. Also because of the upper bound, Lemma 3.1k, for $I_{\epsilon}\left(u_{\epsilon}\right)$, something of order $O\left(\epsilon^{2 / 3}\right)$, we find that $p_{i}^{3}=O\left(\epsilon^{2 / 3}\right)$ and $q_{i}^{3}=O\left(\epsilon^{2 / 3}\right)$.

Sum over $i$ in Lemmas 5.1 and 5.2 to obtain our first lower bound of $I_{\epsilon}\left(u_{\epsilon}\right)$.

LEMMA 5.4

$$
\begin{aligned}
I_{\epsilon}\left(u_{\epsilon}\right) \geqslant & c_{0} \epsilon N_{\epsilon}+\sum_{i=1}^{M_{\epsilon}+1} p_{i} O\left(\mathrm{e}^{-\mu p_{i} / \epsilon}\right)+\sum_{i=1}^{M_{\epsilon}} q_{i} O\left(\mathrm{e}^{-\mu q_{i} / \epsilon}\right) \\
& +\frac{(1-m)^{2}}{24}\left[4 p_{1}^{3}+\sum_{i=2}^{M_{\epsilon}} p_{i}^{3}+4 p_{M_{\epsilon}+1}^{3}\right]+\frac{(1+m)^{2}}{24} \sum_{i=1}^{M_{\epsilon}} q_{i}^{3} \\
& +\left[\sum_{i=1}^{M_{\epsilon}+1} p_{i}^{3}+\sum_{i=1}^{M_{\epsilon}} q_{i}^{3}\right] O\left(\epsilon^{1 / 3}\right)+\left[\sum_{i=1}^{M_{\epsilon}+1} p_{i}^{2}+\sum_{i=1}^{M_{\epsilon}} q_{i}^{2}\right] O(\epsilon) .
\end{aligned}
$$

An important consequence of Lemma 5.4 is that $1 / N_{\epsilon} \sim \epsilon^{1 / 3}$. We need a simple technical lemma first.

Lemma 5.5 1. In the set $\left\{\left(p_{1}, \ldots, p_{M+1}\right): p_{i}>0, i=1, \ldots, M+1, p_{1}+\cdots+p_{M+1}=d\right.$, $d>0\}, 4 p_{1}^{3}+\sum_{i=2}^{M} p_{i}^{3}+4 p_{M+1}^{3}$ is minimized when $2 p_{1}, p_{2}, \ldots, p_{M}$, and $2 p_{M+1}$ are all equal to $p=d / M$. Moreover

$$
\begin{aligned}
4 p_{1}^{3}+\sum_{i=2}^{M} p_{i}^{3}+4 p_{M+1}^{3} \geqslant & 4\left(\frac{p}{2}\right)^{3}+\sum_{i=2}^{M} p^{3}+4\left(\frac{p}{2}\right)^{3}+4 p\left(p_{1}-\frac{p}{2}\right)^{2} \\
& +\sum_{i=2}^{M} 2 p\left(p_{i}-p\right)^{2}+4 p\left(p_{M+1}-\frac{p}{2}\right)^{2} .
\end{aligned}
$$


2. In the set $\left\{\left(q_{1}, \ldots, q_{M}\right): q_{i}>0, i=1, \ldots, M, q_{1}+\cdots+q_{M}=d, d>0\right\}, \sum_{i=1}^{M} q_{i}^{3}$ is minimized when $q_{1}, \ldots, q_{M}$ are all equal to $q=d / M$. Moreover

$$
\sum_{i=1}^{M} q_{i}^{3} \geqslant \sum_{i=1}^{M} q^{3}+\sum_{i=1}^{M} 2 q\left(q_{i}-q\right)^{2}
$$

Proof. We only treat case 1 . Note that

$$
p_{i}^{3}=p^{3}+3 p^{2}\left(p_{i}-p\right)+2 p\left(p_{i}-p\right)^{2}+p_{i}\left(p_{i}-p\right)^{2} \geqslant p^{3}+3 p^{2}\left(p_{i}-p\right)+2 p\left(p_{i}-p\right)^{2}
$$

when $i \neq 1, M+1$. And when $i=1$ or $M+1$,

$$
\begin{aligned}
4 p_{i}^{3} & =4\left(\frac{p}{2}\right)^{3}+3 p^{2}\left(p_{i}-\frac{p}{2}\right)+4 p\left(p_{i}-\frac{p}{2}\right)^{2}+4 p_{i}\left(p_{i}-\frac{p}{2}\right)^{2} \\
& \geqslant 4\left(\frac{p}{2}\right)^{3}+3 p^{2}\left(p_{i}-\frac{p}{2}\right)+4 p\left(p_{i}-\frac{p}{2}\right)^{2} .
\end{aligned}
$$

The lemma then follows after we sum over $i$.

We also need the facts that

$$
\sum_{i=1}^{M_{\epsilon}+1} p_{i}=\frac{1+m}{2}+O\left(\epsilon^{1 / 3}+\epsilon N_{\epsilon}\right), \quad \sum_{i=1}^{M_{\epsilon}} q_{i}=\frac{1-m}{2}+O\left(\epsilon^{1 / 3}+\epsilon N_{\epsilon}\right) .
$$

To see (5.3) we note that

$$
m=\int_{0}^{1} u_{\epsilon} \mathrm{d} x=\sum_{i=1}^{M_{\epsilon}+1} \int_{p_{i}} u_{\epsilon} \mathrm{d} x+\sum_{i=1}^{M_{\epsilon}} \int_{q_{i}} u_{\epsilon} \mathrm{d} x
$$

Every $p_{i}$ or $q_{i}$ interval is further divided in the middle, except the end intervals. Then, for example, with $U_{\epsilon}(t)=u_{\epsilon}\left(\epsilon t+x_{2 i-2}\right)$,

$$
\begin{aligned}
\int_{x_{2 i-2}}^{x_{2 i-2}+p_{i} / 2} u_{\epsilon} \mathrm{d} x & =\epsilon \int_{0}^{p_{i} /(2 \epsilon)} U_{\epsilon} \mathrm{d} t \\
& =\epsilon \int_{0}^{p_{i} /(2 \epsilon)}\left(U_{\epsilon}-H\right) \mathrm{d} t+\epsilon \int_{0}^{p_{i} /(2 \epsilon)}(H-1) \mathrm{d} t+\frac{p_{i}}{2} .
\end{aligned}
$$

The first term of the last line is of order

$$
p_{i} O\left(\epsilon^{1 / 3}\right)+p_{i} O\left(\mathrm{e}^{-v p_{i} / \epsilon}\right)=p_{i} O\left(\epsilon^{1 / 3}\right)+\epsilon O\left(\frac{p_{i}}{\epsilon} \mathrm{e}^{-v p_{i} / \epsilon}\right)=p_{i} O\left(\epsilon^{1 / 3}\right)+o(\epsilon)
$$

by Lemma 4.6 The second term is of order $O(\epsilon)$, because $|H-1|$ is integrable on $(0, \infty)$. Summing over all the $p_{i}$ and $q_{i}$ intervals, we deduce

$$
\sum_{i=1}^{M_{\epsilon}+1} p_{i}-\sum_{i=1}^{M_{\epsilon}} q_{i}=m+O\left(\epsilon^{1 / 3}\right)+O\left(\epsilon N_{\epsilon}\right)
$$


On the other hand,

$$
\sum_{i=1}^{M_{\epsilon}+1} p_{i}+\sum_{i=1}^{M_{\epsilon}} q_{i}=1
$$

(5.3) follows after we solve these two equations.

Proposition $5.61 / N_{\epsilon} \sim \epsilon^{1 / 3}$.

Proof. We only need a weaker version of Lemma 5.4 Note that

$$
\sum_{i=1}^{M_{\epsilon}} p_{i} O\left(\mathrm{e}^{-\mu p_{i} / \epsilon}\right)=\epsilon \sum_{i=1}^{M_{\epsilon}} \frac{p_{i}}{\epsilon} O\left(\mathrm{e}^{-\mu p_{i} / \epsilon}\right)=N_{\epsilon} O(\epsilon)
$$

since $\left(p_{i} / \epsilon\right) O\left(\mathrm{e}^{-\mu p_{i} / \epsilon}\right)=o(1)$. By Proposition 4.5 .

$$
p_{i}^{3} O\left(\epsilon^{1 / 3}\right)+p_{i}^{2} O(\epsilon)=p_{i}^{3}\left(O\left(\epsilon^{1 / 3}\right)+\frac{\epsilon}{p_{i}} O(1)\right)=p_{i}^{3} o(1) .
$$

Then by Lemma 5.4

$$
\begin{aligned}
I_{\epsilon}\left(u_{\epsilon}\right) \geqslant & c_{0} \epsilon N_{\epsilon}+N_{\epsilon} o(\epsilon)+\frac{(1-m)^{2}}{24}\left[4 p_{1}^{3}+\sum_{i=2}^{M_{\epsilon}} p_{i}^{3}+4 p_{M_{\epsilon}+1}^{3}\right] \\
& +\frac{(1+m)^{2}}{24} \sum_{i=1}^{M_{\epsilon}} q_{i}^{3}+\left[\sum_{i=1}^{M_{\epsilon}+1} p_{i}^{3}+\sum_{i=1}^{M_{\epsilon}} q_{i}^{3}\right] o(1) \\
= & c_{0} \epsilon N_{\epsilon}+N_{\epsilon} o(\epsilon)+\left(\frac{(1-m)^{2}}{24}+o(1)\right)\left[4 p_{1}^{3}+\sum_{i=1}^{M_{\epsilon}} p_{i}^{3}+4 p_{M_{\epsilon}+1}^{3}\right] \\
& +\left(\frac{(1+m)^{2}}{24}+o(1)\right) \sum_{i=1}^{M_{\epsilon}} q_{i}^{3} .
\end{aligned}
$$

According to Lemma 5.5 and $5.3,4 p_{1}^{3}+p_{2}^{3}+\cdots+4 p_{M_{\epsilon}+1}^{3}$ achieves its minimum if all $2 p_{1}, p_{2}$, $\ldots, 2 p_{M_{\epsilon}+1}$ happen to be

$$
p=\frac{1}{M_{\epsilon}} \sum_{i=1}^{M_{\epsilon}+1} p_{i}=\frac{\frac{1+m}{2}+O\left(\epsilon^{1 / 3}+\epsilon N_{\epsilon}\right)}{M_{\epsilon}}=\frac{\frac{1+m}{2}+o(1)}{M_{\epsilon}} .
$$

Therefore

$$
4 p_{1}^{3}+\sum_{i=2}^{M_{\epsilon}} p_{i}^{3}+4 p_{M_{\epsilon}+1}^{3} \geqslant M_{\epsilon}^{-2}\left(\frac{1+m}{2}+o(1)\right)^{3} .
$$

After applying the same argument to $q_{i}$, we deduce from 5.4 that

$$
\begin{aligned}
I_{\epsilon}\left(u_{\epsilon}\right) \geqslant & c_{0} \epsilon N_{\epsilon}+N_{\epsilon} o(\epsilon)+\frac{(1-m)^{2}}{24}\left(\frac{1+m}{2}\right)^{3} M_{\epsilon}^{-2} \\
& +\frac{(1+m)^{2}}{24}\left(\frac{1-m}{2}\right)^{3} M_{\epsilon}^{-2}+M_{\epsilon}^{-2} o(1) \\
= & c_{0} \epsilon N_{\epsilon}+N_{\epsilon} o(\epsilon)+\frac{\left(1-m^{2}\right)^{2}}{24 N_{\epsilon}^{2}}+N_{\epsilon}^{-2} o(1) .
\end{aligned}
$$


Recall the upper bound, Lemma 3.1 , for $I_{\epsilon}\left(u_{\epsilon}\right)$. We find

$$
c_{0} \epsilon N_{\epsilon}+N_{\epsilon} o(\epsilon)+\frac{\left(1-m^{2}\right)^{2}}{24 N_{\epsilon}^{2}}+N_{\epsilon}^{-2} o(1)=O\left(\epsilon^{2 / 3}\right) .
$$

Therefore

$$
N_{\epsilon}=O\left(\epsilon^{-1 / 3}\right), \quad N_{\epsilon}^{-2}=O\left(\epsilon^{2 / 3}\right),
$$

which completes the proof.

\section{The first estimation of $p_{i}$ and $q_{i}$}

The crude lower and upper bounds for $p_{i}$ and $q_{i}$ in Propositions 4.5 and 5.3 are improved in this and the next sections. The upper bound is lowered to $O\left(\epsilon^{1 / 3}\right)$ first. To prove this we have to treat long $p_{i}$ and $q_{i}$ intervals and possible short $p_{i}$ and $q_{i}$ intervals differently. Let $c_{2}$ be a positive number large enough so that when $p_{i} \geqslant-c_{2} \epsilon \log \epsilon$,

$$
p_{i} \mathrm{e}^{-\mu p_{i} / \epsilon}=O\left(\epsilon^{13 / 9}\right), \quad \mathrm{e}^{-v p_{i} / \epsilon}=o\left(\epsilon^{1 / 3}\right) .
$$

When $p_{i}$ (or $q_{i}$ ) is not an end interval, we say $p_{i}$ (or $q_{i}$ ) is long if $p_{i} \geqslant-c_{2} \epsilon \log \epsilon$ (or $q_{i} \geqslant$ $-c_{2} \epsilon \log \epsilon$ ). When $p_{i}$ (or $q_{i}$ ) is an end interval, we say $p_{i}$ (or $q_{i}$ ) is long if $p_{i} \geqslant-\left(c_{2} / 2\right) \epsilon \log \epsilon$ (or $q_{i} \geqslant-\left(c_{2} / 2\right) \epsilon \log \epsilon$ ). Otherwise we say $p_{i}$ (or $q_{i}$ ) is short. Let $P_{L}$ and $P_{S}$ be the numbers of long and short $p_{i}$ intervals respectively, and $Q_{L}$ and $Q_{S}$ be the numbers of long and short $q_{i}$ intervals respectively. Here we count an end interval as $1 / 2$, so $P_{L}, P_{S}, Q_{L}, Q_{S}$ are integers or half-integers.

In the next section we will show that short intervals do not exist (see (7.6)).

Proposition $6.1 p_{i}=O\left(\epsilon^{1 / 3}\right)$ and $q_{i}=O\left(\epsilon^{1 / 3}\right)$.

Proof. On a short $p_{i}$ or $q_{i}$ interval we ignore the nonlocal part of the energy and use Lemma 5.1 to obtain

$\int_{p_{i}}\left[\frac{\epsilon^{2}}{2}\left|u_{\epsilon}^{\prime}\right|^{2}+W\left(u_{\epsilon}\right)+\frac{1}{2}\left|v_{\epsilon}^{\prime}\right|^{2}\right] \mathrm{d} x \geqslant c_{1} \epsilon, \quad \int_{q_{i}}\left[\frac{\epsilon^{2}}{2}\left|u_{\epsilon}^{\prime}\right|^{2}+W\left(u_{\epsilon}\right)+\frac{1}{2}\left|v_{\epsilon}^{\prime}\right|^{2}\right] \mathrm{d} x \geqslant c_{-1} \epsilon$.

Here we have again used the fact that

$$
2 c_{1} \epsilon+p_{i} O\left(\mathrm{e}^{-\mu p_{i} / \epsilon}\right)=2 c_{1} \epsilon+\epsilon O\left(\frac{p_{i}}{\epsilon} \mathrm{e}^{-\mu p_{i} / \epsilon}\right)=2 c_{1} \epsilon+o(\epsilon) \geqslant c_{1} \epsilon
$$

when $\epsilon$ is small. If an end interval, $p_{1}$ or $p_{M_{\epsilon}+1}$, happens to be short, replace $c_{1} \epsilon$ by $\left(c_{1} / 2\right) \epsilon$ in 6.2 .

On a long interval we note by Proposition 5.3 that $O(\epsilon) p_{i}^{2}$ which appears in Lemma 5.2 is $O\left(\epsilon^{13 / 9}\right)$. Then by Lemmas 5.15 .2 and the definition 6.1 of long intervals,

$$
\begin{aligned}
\int_{p_{i}}\left[\frac{\epsilon^{2}}{2}\left|u_{\epsilon}^{\prime}\right|^{2}+W\left(u_{\epsilon}\right)\right. & \left.+\frac{1}{2}\left|v_{\epsilon}^{\prime}\right|^{2}\right] \mathrm{d} x \\
& \geqslant 2 c_{1} \epsilon+O\left(\mathrm{e}^{-\mu p_{i} / \epsilon}\right)+\left[\frac{(1-m)^{2}}{24}+O\left(\epsilon^{1 / 3}\right)\right] p_{i}^{3}+O(\epsilon) p_{i}^{2} \\
& =2 c_{1} \epsilon+\left[\frac{(1-m)^{2}}{24}+O\left(\epsilon^{1 / 3}\right)\right] p_{i}^{3}+O\left(\epsilon^{13 / 9}\right),
\end{aligned}
$$




$$
\int_{q_{i}}\left[\frac{\epsilon^{2}}{2}\left|u_{\epsilon}^{\prime}\right|^{2}+W\left(u_{\epsilon}\right)+\frac{1}{2}\left|v_{\epsilon}^{\prime}\right|^{2}\right] \mathrm{d} x \geqslant 2 c_{-1} \epsilon+\left[\frac{(1+m)^{2}}{24}+O\left(\epsilon^{1 / 3}\right)\right] q_{i}^{3}+O\left(\epsilon^{13 / 9}\right) .
$$

If $p_{i}$ happens to be the end interval $p_{1}$ or $p_{M_{\epsilon}+1}$, then $(6.3)$ is replaced by

$$
c_{1} \epsilon+\left[\frac{(1-m)^{2}}{6}+O\left(\epsilon^{1 / 3}\right)\right] p_{i}^{3}+O\left(\epsilon^{13 / 9}\right)
$$

Sum (6.2) through (6.4) over $i$ :

$$
\begin{aligned}
I_{\epsilon}\left(u_{\epsilon}\right) \geqslant & c_{1} \epsilon P_{S}+c_{-1} \epsilon Q_{S}+O\left(\epsilon^{10 / 9}\right) \\
& +\sum_{i: \text { long }}\left\{2 c_{1} \epsilon+\left[\frac{(1-m)^{2}}{24}+O\left(\epsilon^{1 / 3}\right)\right] p_{i}^{3}\right\} \\
& +\sum_{i: \text { long }}\left\{2 c_{-1} \epsilon+\left[\frac{(1+m)^{2}}{24}+O\left(\epsilon^{1 / 3}\right)\right] q_{i}^{3}\right\},
\end{aligned}
$$

where $O\left(\epsilon^{10 / 9}\right)$ follows from $\left(P_{L}+Q_{L}\right) O\left(\epsilon^{13 / 9}\right)=N_{\epsilon} O\left(\epsilon^{13 / 9}\right)=O\left(\epsilon^{10 / 9}\right)$ by Proposition 5.6. Again if $i$ in the first sum of the last inequality happens to be 1 or $M_{\epsilon}+1$, the quantity in the sum should read the first two terms of 6.5 .

Note that with Proposition 5.6, (5.3) is simplified to

$$
\sum_{i=1}^{M_{\epsilon}+1} p_{i}=\frac{1+m}{2}+O\left(\epsilon^{1 / 3}\right), \quad \sum_{i=1}^{M_{\epsilon}} q_{i}=\frac{1-m}{2}+O\left(\epsilon^{1 / 3}\right)
$$

Therefore

$$
\sum_{i: \text { long }} p_{i}=\frac{1+m}{2}+O\left(\epsilon^{1 / 3}\right), \quad \sum_{i: \text { long }} q_{i}=\frac{1-m}{2}+O\left(\epsilon^{1 / 3}\right)
$$

since

$$
\sum_{i: \text { long }} p_{i}=\sum_{i=1}^{M_{\epsilon}+1} p_{i}-\sum_{i: \text { short }} p_{i}=\frac{1+m}{2}+O\left(\epsilon^{1 / 3}\right)+N_{\epsilon} O(-\epsilon \log \epsilon) .
$$

We again use Lemma 5.5 to deduce, using the same convention when an end interval is involved,

$$
\begin{aligned}
I_{\epsilon}\left(u_{\epsilon}\right) \geqslant & c_{1} \epsilon P_{S}+c_{-1} \epsilon Q_{S}+O\left(\epsilon^{10 / 9}\right) \\
& +\sum_{i: \text { long }}\left\{2 c_{1} \epsilon+\left[\frac{(1-m)^{2}}{24}+O\left(\epsilon^{1 / 3}\right)\right] p^{3}\right\} \\
& +\sum_{i: \text { long }}\left\{2 c_{1} \epsilon+\left[\frac{(1+m)^{2}}{24}+O\left(\epsilon^{1 / 3}\right)\right] q^{3}\right\} \\
\geqslant & c_{1} \epsilon P_{S}+c_{-1} \epsilon Q_{S}+O\left(\epsilon^{10 / 9}\right)+2 c_{1} \epsilon P_{L}+2 c_{-1} \epsilon Q_{L} \\
& +P_{L}^{-2}\left[\frac{(1-m)^{2}}{24}+O\left(\epsilon^{1 / 3}\right)\right]\left(\frac{1+m}{2}+O\left(\epsilon^{1 / 3}\right)\right)^{3} \\
& +Q_{L}^{-2}\left[\frac{(1+m)^{2}}{24}+O\left(\epsilon^{1 / 3}\right)\right]\left(\frac{1-m}{2}+O\left(\epsilon^{1 / 3}\right)\right)^{3},
\end{aligned}
$$


where the last step follows from 6.8 and

$$
p=P_{L}^{-1} \sum_{i: \text { long }} p_{i}, \quad q=Q_{L}^{-1} \sum_{i: \text { long }} q_{i} .
$$

The upper bound of $I_{\epsilon}\left(u_{\epsilon}\right)$, Lemma 3.1 , then implies

$$
2 c_{1} \epsilon P_{L}=O\left(\epsilon^{2 / 3}\right), \quad P_{L}^{-2}\left[\frac{(1-m)^{2}}{24}+O\left(\epsilon^{1 / 3}\right)\right]\left(\frac{1-m}{2}+O\left(\epsilon^{1 / 3}\right)\right)^{3}=O\left(\epsilon^{2 / 3}\right) .
$$

Therefore, after applying a similar argument to $q_{i}$, we find

$$
P_{L} \sim \epsilon^{-1 / 3}, \quad Q_{L} \sim \epsilon^{-1 / 3} .
$$

6.10) in turn simplifies 6.9 to

$$
\begin{aligned}
I_{\epsilon}\left(u_{\epsilon}\right) \geqslant & c_{1} \epsilon P_{S}+c_{-1} \epsilon Q_{S}+O(\epsilon) \\
& +2 c_{1} \epsilon P_{L}+P_{L}^{-2} \frac{(1-m)^{2}}{24}\left(\frac{1+m}{2}\right)^{3} \\
& +2 c_{-1} \epsilon Q_{L}+Q_{L}^{-2} \frac{(1+m)^{2}}{24}\left(\frac{1-m}{2}\right)^{3} .
\end{aligned}
$$

Now the mysterious definition (2.5) of $\alpha$ comes into play. Relation (2.7) implies that the last two lines in (6.11) are proportional. They are simultaneously minimized if $P_{L}$ and $Q_{L}$ happen to be the integer or half-integer that minimizes them. Denote this integer or half-integer by $R_{\epsilon}$. As in Lemma $3.12, R_{\epsilon} \sim \epsilon^{-1 / 3}$. Then we deduce from 6.11, replacing both $P_{L}$ and $Q_{L}$ by $R_{\epsilon}$,

$$
I_{\epsilon}\left(u_{\epsilon}\right) \geqslant c_{1} \epsilon P_{S}+c_{-1} \epsilon Q_{S}+O(\epsilon)+2 c_{0} \epsilon R_{\epsilon}+\frac{\left(1-m^{2}\right)^{2}}{96 R_{\epsilon}^{2}} \text {. }
$$

Now use $N=2 R_{\epsilon}$ in Lemma 3.1, to obtain an upper bound

$$
I_{\epsilon}\left(u_{\epsilon}\right) \leqslant 2 c_{0} \epsilon R_{\epsilon}+\frac{\left(1-m^{2}\right)^{2}}{96 R_{\epsilon}^{2}}+O\left(\epsilon^{4 / 3} \log \epsilon\right),
$$

which, combined with 6.12, gives $c_{1} \epsilon P_{S}+c_{-1} \epsilon Q_{S}=O(\epsilon)$. Therefore

$$
P_{S}=O(1), \quad Q_{S}=O(1) .
$$

We now revisit (6.6) with the full power of Lemma 5.5 Because we know from 6.10) that

$$
p \sim \epsilon^{1 / 3}, \quad q \sim \epsilon^{1 / 3},
$$

and also because of 6.10 and 6.13 , using them to handle the error terms we find that 6.6 yields

$$
\begin{aligned}
I_{\epsilon}\left(u_{\epsilon}\right) \geqslant & O(\epsilon)+\sum_{i: \text { long }}\left\{2 c_{1} \epsilon+\left[\frac{(1-m)^{2}}{24}+O\left(\epsilon^{1 / 3}\right)\right] p_{i}^{3}\right\} \\
& +\sum_{i: \text { long }}\left\{2 c_{1} \epsilon+\left[\frac{(1+m)^{2}}{24}+O\left(\epsilon^{1 / 3}\right)\right] q_{i}^{3}\right\}
\end{aligned}
$$




$$
\begin{aligned}
\geqslant & O(\epsilon)+\sum_{i: \text { long }}\left\{2 c_{1} \epsilon+\left[\frac{(1-m)^{2}}{24}+O\left(\epsilon^{1 / 3}\right)\right]\left[p^{3}+2 p\left(p_{i}-p\right)^{2}\right]\right\} \\
& +\sum_{i: \text { long }}\left\{2 c_{1} \epsilon+\left[\frac{(1+m)^{2}}{24}+O\left(\epsilon^{1 / 3}\right)\right]\left[q^{3}+2 q\left(q_{i}-q\right)^{2}\right]\right\} \\
= & O(\epsilon)+2 c_{1} \epsilon P_{L}+P_{L}^{-2} \frac{(1-m)^{2}}{24}\left(\frac{1+m}{2}\right)^{3} \\
& +2 c_{-1} \epsilon Q_{L}+Q_{L}^{-2} \frac{(1+m)^{2}}{24}\left(\frac{1-m}{2}\right)^{3} \\
& +2 \sum_{i: \text { long }}\left[\frac{(1+m)^{2}}{24}+O\left(\epsilon^{1 / 3}\right)\right] p\left(p_{i}-p\right)^{2} \\
& +2 \sum_{i: \text { long }}\left[\frac{(1-m)^{2}}{24}+O\left(\epsilon^{1 / 3}\right)\right] q\left(q_{i}-q\right)^{2} .
\end{aligned}
$$

If $p_{i}$ is an end interval $p_{1}$ or $p_{M_{\epsilon}+1}$, then in the second last line $p\left(p_{i}-p\right)$ should read $2 p\left(p_{i}-p / 2\right)$. We again replace $P_{L}$ and $Q_{L}$ by $R_{\epsilon}$, introduced before 6.12), to have a quantity less than or equal to $I_{\epsilon}\left(u_{\epsilon}\right)$. Also take $N=2 R_{\epsilon}$ in Lemma 3.1 to bound $I_{\epsilon}\left(u_{\epsilon}\right)$ from above. Combining these two bounds, as in the argument before 6.13, we obtain

$$
O(\epsilon)+\sum_{i: \text { long }} \frac{(1+m)^{2}}{24} p\left(p_{i}-p\right)^{2}+\sum_{i: \text { long }} \frac{(1-m)^{2}}{24} q\left(q_{i}-q\right)^{2} \leqslant O\left(\epsilon^{4 / 3} \log \epsilon\right)
$$

i.e.

$$
\sum_{i: \text { long }} \frac{(1+m)^{2}}{24} p\left(p_{i}-p\right)^{2}+\sum_{i: \text { long }} \frac{(1-m)^{2}}{24} q\left(q_{i}-q\right)^{2}=O(\epsilon)
$$

which implies $p\left(p_{i}-p\right)^{2}=O(\epsilon), q\left(q_{i}-q\right)^{2}=O(\epsilon)$. The proposition follows since $p \sim \epsilon^{1 / 3}$ and $q \sim \epsilon^{1 / 3}$, by 6.14 .

With the help of Proposition 6.1. Lemma 4.6 is sharpened to

Lemma 6.2 1. For $i=2, \ldots, M_{\epsilon}+1$, if the $q_{i-1}$ interval before $x_{2 i-2}$ and the $p_{i}$ interval after $x_{2 i-2}$ are both long then

$$
\begin{aligned}
\left\|u_{\epsilon}\left(\epsilon t+x_{2 i-2}\right)-H(t)\right\|_{C^{2}\left[0, p_{i} /(2 \epsilon)\right]} & =o\left(\epsilon^{1 / 3}\right), \\
\left\|u_{\epsilon}\left(\epsilon t+x_{2 i-2}\right)-H(t)\right\|_{C^{2}\left[-q_{i-1} /(2 \epsilon), 0\right]} & =o\left(\epsilon^{1 / 3}\right) .
\end{aligned}
$$

2. For $i=1, \ldots, M_{\epsilon}$, if the $p_{i}$ interval before $x_{2 i-1}$ and the $q_{i}$ interval after $x_{2 i-1}$ are both long then

$$
\begin{aligned}
\left\|u_{\epsilon}\left(\epsilon t+x_{2 i-1}\right)-H(-t)\right\|_{C^{2}\left[0, q_{i} /(2 \epsilon)\right]} & =o\left(\epsilon^{1 / 3}\right) \\
\left\|u_{\epsilon}\left(\epsilon t+x_{2 i-1}\right)-H(-t)\right\|_{C^{2}\left[-p_{i} /(2 \epsilon), 0\right]} & =o\left(\epsilon^{1 / 3}\right) .
\end{aligned}
$$


Proof. It follows from $\int_{0}^{1}\left|v_{\epsilon}^{\prime}\right|^{2}=O\left(\epsilon^{2 / 3}\right)$ and $v_{\epsilon}^{\prime \prime}=O(1)$ that $v_{\epsilon}^{\prime}=o(1)$ on $(0,1)$. Let $x_{2 i-2}$ be an $\alpha$-point between two long intervals $q_{i-1}$ and $p_{i}$. For every $x \in(a, b):=\left(x_{2 i-2}-q_{i-1} / 2, x_{2 i-2}+\right.$ $\left.p_{i} / 2\right)$, by Proposition 6.1 .

$$
v_{\epsilon}(x)=v_{\epsilon}\left(x_{2 i-2}\right)+\int_{x_{2 i-2}}^{x} v_{\epsilon}^{\prime} \mathrm{d} x=v_{\epsilon}\left(x_{2 i-2}\right)+o(1) \cdot O\left(\epsilon^{1 / 3}\right)=v_{\epsilon}\left(x_{2 i-2}\right)+o\left(\epsilon^{1 / 3}\right) .
$$

Let $u_{\epsilon}=w_{\epsilon}+\phi_{\epsilon}$ where $w_{\epsilon}=H\left(\left(x-x_{2 i-2}\right) / \epsilon\right)$. Lemma 4.6 and the definition 6.1 of long intervals imply that

$$
\left\|\phi_{\epsilon}\left(\epsilon t+x_{2 i-2}\right)\right\|_{C^{2}[a, b]}=O\left(\epsilon^{1 / 3}\right)+O\left(\mathrm{e}^{-v p_{i} / \epsilon}\right)+O\left(\mathrm{e}^{-v q_{i-1} / \epsilon}\right)=O\left(\epsilon^{1 / 3}\right) .
$$

Rewrite $(1.5)$ as

$$
-\epsilon^{2}\left(w_{\epsilon}^{\prime \prime}+\phi_{\epsilon}^{\prime \prime}\right)+f\left(w_{\epsilon}\right)+f^{\prime}\left(w_{\epsilon}\right) \phi_{\epsilon}+\frac{1}{2} f^{\prime \prime}(\ldots) \phi_{\epsilon}^{2}+v_{\epsilon}\left(x_{2 i-2}\right)-\lambda_{\epsilon}+o\left(\epsilon^{1 / 3}\right)=0,
$$

which is simplified to

$$
-\epsilon^{2} \phi_{\epsilon}^{\prime \prime}+f^{\prime}\left(w_{\epsilon}\right) \phi_{\epsilon}+v_{\epsilon}\left(x_{2 i-2}\right)-\lambda_{\epsilon}+o\left(\epsilon^{1 / 3}\right)=0
$$

if we use 6.17 for $\phi_{\epsilon}$ in the $f^{\prime \prime}$ term. Multiply this equation by $w_{\epsilon}^{\prime}$ and integrate over $(a, b)$ :

$$
\int_{a}^{b}\left[-\epsilon^{2} \phi_{\epsilon}^{\prime \prime} w_{\epsilon}^{\prime}+f^{\prime}\left(w_{\epsilon}\right) \phi_{\epsilon}^{\prime} w_{\epsilon}^{\prime}\right]+\int_{a}^{b}\left[v_{\epsilon}\left(x_{2 i-2}\right)-\lambda_{\epsilon}+o\left(\epsilon^{1 / 3}\right)\right] w_{\epsilon}^{\prime}=0 .
$$

Then integrate by parts to get

$$
\left.\left(-\epsilon^{2} \phi_{\epsilon}^{\prime} w_{\epsilon}^{\prime}+\epsilon^{2} \phi_{\epsilon} w_{\epsilon}^{\prime \prime}\right)\right|_{x=a} ^{x=b}+\left[v_{\epsilon}\left(x_{2 i-2}\right)-\lambda_{\epsilon}+o\left(\epsilon^{1 / 3}\right)\right](2+o(1))=0 .
$$

Use 6.17) again to deduce

$$
\begin{aligned}
{\left[v_{\epsilon}\left(x_{i}\right)\right.} & \left.-\lambda_{\epsilon}+o\left(\epsilon^{1 / 3}\right)\right](2+o(1)) \\
& =-\left[-O\left(\epsilon^{2} \epsilon^{1 / 3} \epsilon^{-1} \epsilon^{-1}\right) H^{\prime}\left(\frac{x-x_{2 i-2}}{\epsilon}\right)+O\left(\epsilon^{2} \epsilon^{1 / 3} \epsilon^{-2}\right) H^{\prime \prime}\left(\frac{x-x_{2 i-2}}{\epsilon}\right)\right]_{x=a}^{x=b} \\
& =O\left(\epsilon^{1 / 3}\right) \cdot o(1)+O\left(\epsilon^{1 / 3}\right) \cdot o(1)=o\left(\epsilon^{1 / 3}\right) .
\end{aligned}
$$

Therefore $v_{\epsilon}\left(x_{i}\right)-\lambda_{\epsilon}=o\left(\epsilon^{1 / 3}\right)$. Combining this with 6.16), we deduce that on $(0,1), v_{\epsilon}-\lambda_{\epsilon}=$ $o\left(\epsilon^{1 / 3}\right)$ and $u_{\epsilon}$ satisfies $-\epsilon^{2} u_{\epsilon}^{\prime \prime}+f\left(u_{\epsilon}\right)=o\left(\epsilon^{1 / 3}\right)$.

Now we follow the proof of Lemma 4.6, with all the $O\left(\epsilon^{1 / 3}\right), O\left(\mathrm{e}^{-v p_{i} / \epsilon}\right)$ and $O\left(\mathrm{e}^{-v q_{i}} \epsilon\right)$ terms replaced by $o\left(\epsilon^{1 / 3}\right)$, to complete the proof of this lemma.

This upgrade to Lemma 4.6 gives us a much needed improvement of the lower bound in Lemma5.2

Lemma 6.3 On a long $p_{i}$ ( $q_{i}$ respectively) interval which is not adjacent (to the left or right) to a short interval, the nonlocal part of $I_{\epsilon}\left(u_{\epsilon}\right)$ has the lower bound

$$
\frac{1}{2} \int_{p_{i}}\left|v_{\epsilon}^{\prime}\right|^{2} \geqslant \frac{(1-m)^{2}}{6} p_{i}^{3}+o\left(\epsilon^{4 / 3}\right), \quad i=1, M_{\epsilon}+1,
$$




$$
\begin{aligned}
& \frac{1}{2} \int_{p_{i}}\left|v_{\epsilon}^{\prime}\right|^{2} \geqslant \frac{(1-m)^{2}}{24} p_{i}^{3}+\frac{p_{i}}{2}\left[v_{\epsilon}^{\prime}\left(x_{2 i-2}\right)-\frac{(1-m) p_{i}}{2}+o\left(\epsilon^{2 / 3}\right)\right]^{2}+o\left(\epsilon^{4 / 3}\right), \\
& i \neq 1, M_{\epsilon}+1 \\
& \frac{1}{2} \int_{q_{i}}\left|v_{\epsilon}^{\prime}\right|^{2} \geqslant \frac{(1+m)^{2}}{24} q_{i}^{3}+\frac{q_{i}}{2}\left[v_{\epsilon}^{\prime}\left(x_{2 i-1}\right)+\frac{(1+m) q_{i}}{2}+o\left(\epsilon^{2 / 3}\right)\right]^{2}+o\left(\epsilon^{4 / 3}\right) .
\end{aligned}
$$

Proof. We follow the proof of Lemma 5.2 with all the $O\left(\epsilon^{1 / 3}\right)$ 's replaced by $o\left(\epsilon^{1 / 3}\right)$, using Proposition 6.1 along the way to simplify error terms.

As pointed out in the second remark following the proof of Lemma 5.2 there is another version of Lemma 6.3 analogous to 5.2 :

$$
\begin{aligned}
& \frac{1}{2} \int_{p_{i}}\left|v_{\epsilon}^{\prime}\right|^{2} \geqslant \frac{(1-m)^{2}}{24} p_{i}^{3}+\frac{p_{i}}{2}\left[v_{\epsilon}^{\prime}\left(x_{2 i+1}\right)+\frac{(1-m) p_{i}}{2}+o\left(\epsilon^{2 / 3}\right)\right]^{2}+o\left(\epsilon^{4 / 3}\right), \\
& i \neq 1, M_{\epsilon}+1, \\
& \frac{1}{2} \int_{q_{i}}\left|v_{\epsilon}^{\prime}\right|^{2} \geqslant \frac{(1+m)^{2}}{24} q_{i}^{3}+\frac{q_{i}}{2}\left[v_{\epsilon}^{\prime}\left(x_{2 i}\right)-\frac{(1+m) q_{i}}{2}+o\left(\epsilon^{2 / 3}\right)\right]^{2}+o\left(\epsilon^{4 / 3}\right) .
\end{aligned}
$$

\section{The second estimation of $p_{i}$ and $q_{i}$}

The goal of this section is to improve Proposition 6.1 to $p_{i} \sim \epsilon^{1 / 3}$ and $q_{i} \sim \epsilon^{1 / 3}$. In particular we need to show that there are no short intervals. When dealing with the end intervals, this section adopts the same convention as in the last section.

We now redo the proof of Proposition 6.1 with this new lower bound, Lemma 6.3, to improve the proposition to

Proposition $7.1 p_{i} \sim \epsilon^{1 / 3}$ and $q_{i} \sim \epsilon^{1 / 3}$.

Proof. We follow the argument in the proof of Proposition 6.1 leading to 6.6, using Lemma 6.3 instead of Lemma 5.2

More specifically on a short interval we use the same estimates 6.2). For a long interval, there are two possibilities: either it is adjacent to a short interval, or it is not. In the first case, we retain the estimates 6.3 and 6.4, which are simplified by Proposition 6.1 to

$$
\begin{aligned}
& \int_{p_{i}}\left[\frac{\epsilon^{2}}{2}\left|u_{\epsilon}^{\prime}\right|^{2}+W\left(u_{\epsilon}\right)+\frac{1}{2}\left|v_{\epsilon}^{\prime}\right|^{2}\right] \mathrm{d} x \geqslant 2 c_{1} \epsilon+\frac{(1-m)^{2}}{24} p_{i}^{3}+O\left(\epsilon^{4 / 3}\right), \\
& \int_{q_{i}}\left[\frac{\epsilon^{2}}{2}\left|u_{\epsilon}^{\prime}\right|^{2}+W\left(u_{\epsilon}\right)+\frac{1}{2}\left|v_{\epsilon}^{\prime}\right|^{2}\right] \mathrm{d} x \geqslant 2 c_{-1} \epsilon+\frac{(1+m)^{2}}{24} q_{i}^{3}+O\left(\epsilon^{4 / 3}\right) .
\end{aligned}
$$

In the second case we apply Lemma 6.3 to obtain

$$
\begin{aligned}
& \int_{p_{i}}\left[\frac{\epsilon^{2}}{2}\left|u_{\epsilon}^{\prime}\right|^{2}+W\left(u_{\epsilon}\right)+\frac{1}{2}\left|v_{\epsilon}^{\prime}\right|^{2}\right] \mathrm{d} x \geqslant 2 c_{1} \epsilon+\frac{(1-m)^{2}}{24} p_{i}^{3}+o\left(\epsilon^{4 / 3}\right), \\
& \int_{q_{i}}\left[\frac{\epsilon^{2}}{2}\left|u_{\epsilon}^{\prime}\right|^{2}+W\left(u_{\epsilon}\right)+\frac{1}{2}\left|v_{\epsilon}^{\prime}\right|^{2}\right] \mathrm{d} x \geqslant 2 c_{-1} \epsilon+\frac{(1+m)^{2}}{24} q_{i}^{3}+o\left(\epsilon^{4 / 3}\right) .
\end{aligned}
$$


As we sum over (6.2), (7.1) and (7.2) we note that there are at most $O(1)$ terms from (7.1) because of 6.13 , and $P_{L} \sim \epsilon^{-1 / 3}$ (and $Q_{L} \sim \epsilon^{-1 / 3}$ by 6.10 ) terms from 2.2 . Therefore

$$
\begin{aligned}
I_{\epsilon}\left(u_{\epsilon}\right) \geqslant & c_{1} \epsilon P_{S}+c_{-1} \epsilon Q_{S}+o(\epsilon)+\sum_{i: \text { long }}\left[2 c_{1} \epsilon+\frac{(1-m)^{2}}{24} p_{i}^{3}\right] \\
& +\sum_{i: \text { long }}\left[2 c_{1} \epsilon+\frac{(1+m)^{2}}{24} q_{i}^{3}\right] .
\end{aligned}
$$

Formula (6.8) needs to be improved as well. Because of $(6.13)$ and the definition of short intervals,

$$
m=\int_{0}^{1} u_{\epsilon} \mathrm{d} x=\sum_{i: \text { long }} \int_{p_{i}} u_{\epsilon} \mathrm{d} x+\sum_{i: \text { long }} \int_{q_{i}} u_{\epsilon} \mathrm{d} x+O(\epsilon \log \epsilon) .
$$

Again every long $p_{i}$ or $q_{i}$ interval is further divided in the middle, except the end intervals. For example, with $U_{\epsilon}(t)=u_{\epsilon}\left(\epsilon t+x_{2 i-2}\right)$,

$$
\begin{aligned}
\int_{x_{2 i-2}}^{x_{2 i-2}+p_{i} / 2} u_{\epsilon} \mathrm{d} x & =\epsilon \int_{0}^{p_{i} /(2 \epsilon)} U_{\epsilon} \mathrm{d} t \\
& =\epsilon \int_{0}^{p_{i} /(2 \epsilon)}\left(U_{\epsilon}-H\right) \mathrm{d} t+\epsilon \int_{0}^{p_{i} /(2 \epsilon)}(H-1) \mathrm{d} t+\frac{p_{i}}{2} .
\end{aligned}
$$

Now if one of the intervals before or after $x_{2 i-2}$ is short, we use the same estimate as in the proof of Proposition 6.1, i.e.

$$
\int_{x_{2 i-2}}^{x_{2 i-2}+p_{i} / 2} u_{\epsilon} \mathrm{d} x=\frac{p_{i}}{2}+p_{i} O\left(\epsilon^{1 / 3}\right)+O(\epsilon)=\frac{p_{i}}{2}+O\left(\epsilon^{2 / 3}\right) .
$$

There are at most $O(1)$ such $x_{2 i-2}$ 's. If neither of the intervals before or after $x_{2 i-2}$ is short, we use Lemma 6.2 to find

$$
\int_{x_{2 i-2}}^{x_{2 i-2}+p_{i} / 2} u_{\epsilon} \mathrm{d} x=p_{i} o\left(\epsilon^{1 / 3}\right)+O(\epsilon)+\frac{p_{i}}{2}=\frac{p_{i}}{2}+o\left(\epsilon^{2 / 3}\right) .
$$

There are $P_{L} \sim \epsilon^{-1 / 3}$ such $x_{2 i-2}$ 's.

Now we sum over all long intervals to find

$$
\sum_{i: \text { long }} p_{i}-\sum_{i: \text { long }} q_{i}=m+o\left(\epsilon^{1 / 3}\right) .
$$

On the other hand, by 6.13,

$$
\sum_{i: \text { long }} p_{i}+\sum_{i: \text { long }} q_{i}=1-\sum_{i: \text { short }} p_{i}-\sum_{i: \text { short }} q_{i}=1-O(1) \cdot O(\epsilon \log \epsilon)=1+o\left(\epsilon^{1 / 3}\right) .
$$

The last two equations imply

$$
\sum_{i: \text { long }} p_{i}=\frac{1+m}{2}+o\left(\epsilon^{1 / 3}\right), \quad \sum_{i: \text { long }} q_{i}=\frac{1-m}{2}+o\left(\epsilon^{1 / 3}\right) .
$$

Again we set

$$
p=P_{L}^{-1} \sum_{i: \text { long }} p_{i}, \quad q=Q_{L}^{-1} \sum_{i: \text { long }} q_{i},
$$


and continue from 7.3 with the help of Lemma 5.5 and (7.4):

$$
\begin{aligned}
I_{\epsilon}\left(u_{\epsilon}\right) \geqslant & c_{1} \epsilon P_{S}+c_{-1} \epsilon Q_{S}+o(\epsilon) \\
& +\sum_{i: \text { long }}\left[2 c_{1} \epsilon+\frac{(1-m)^{2}}{24} p^{3}\right]+\sum_{i: \text { long }}\left[2 c_{1} \epsilon+\frac{(1+m)^{2}}{24} q^{3}\right] \\
\geqslant & c_{1} \epsilon P_{S}+c_{-1} \epsilon Q_{S}+o(\epsilon)+2 c_{1} \epsilon P_{L}+2 c_{-1} \epsilon Q_{L} \\
& +P_{L}^{-2} \frac{(1-m)^{2}}{24}\left(\frac{1+m}{2}+o\left(\epsilon^{1 / 3}\right)\right)^{3} \\
& +Q_{L}^{-2} \frac{(1+m)^{2}}{24}\left(\frac{1-m}{2}+o\left(\epsilon^{1 / 3}\right)\right)^{3} \\
= & c_{1} \epsilon P_{S}+c_{-1} \epsilon Q_{S}+o(\epsilon)+2 c_{1} \epsilon P_{L}+2 c_{-1} \epsilon Q_{L} \\
& +P_{L}^{-2} \frac{(1-m)^{2}}{24}\left(\frac{1+m}{2}\right)^{3}+Q_{L}^{-2} \frac{(1+m)^{2}}{24}\left(\frac{1-m}{2}\right)^{3}
\end{aligned}
$$

where the simplification of error terms to the last two lines uses the estimate 6.10$)$ of $P_{L}$ and $Q_{L}$.

The last quantity is further reduced after we replace $P_{L}$ and $Q_{L}$ both by $R_{\epsilon}$, introduced before 6.12. Also take $N=2 R_{\epsilon}$ in Lemma 3.1, to have an upper bound. Combine these two bounds to deduce

$$
2 c_{0} \epsilon R_{\epsilon}+\frac{\left(1-m^{2}\right)^{2}}{96 R_{\epsilon}^{2}}+O\left(\epsilon^{4 / 3} \log \epsilon\right) \geqslant c_{1} \epsilon P_{S}+c_{-1} \epsilon Q_{S}+o(\epsilon)+2 c_{0} \epsilon R_{\epsilon}+\frac{\left(1-m^{2}\right)^{2}}{96 R_{\epsilon}^{2}},
$$

which leads to $c_{1} \epsilon P_{S}+c_{-1} \epsilon Q_{S}=o(\epsilon)$. Hence

$$
P_{S}=Q_{S}=0
$$

There are no short intervals and $P_{L}=Q_{L}=M_{\epsilon}=N_{\epsilon} / 2 \sim \epsilon^{-1 / 3}$.

Revisit (7.3) to deduce, using (7.4, , 7.6 and Lemma 5.5,

$$
\begin{aligned}
I_{\epsilon}\left(u_{\epsilon}\right) \geqslant & \sum_{i=1}^{M_{\epsilon}+1}\left[2 c_{1} \epsilon+\frac{(1-m)^{2}}{24} p_{i}^{3}\right]+\sum_{i=1}^{M_{\epsilon}}\left[2 c_{-1} \epsilon+\frac{(1+m)^{2}}{24} q_{i}^{3}\right]+o(\epsilon) \\
\geqslant & \sum_{i=1}^{M_{\epsilon}+1}\left[2 c_{1} \epsilon+\frac{(1-m)^{2}}{24} p^{3}\right]+\sum_{i=1}^{M_{\epsilon}}\left[2 c_{-1} \epsilon+\frac{(1+m)^{2}}{24} q^{3}\right]+o(\epsilon) \\
& +\frac{(1-m)^{2}}{24} \sum_{i=1}^{M_{\epsilon}+1} p\left(p_{i}-p\right)^{2}+\frac{(1+m)^{2}}{24} \sum_{i=1}^{M_{\epsilon}} q\left(q_{i}-q\right)^{2} \\
= & o(\epsilon)+2 c_{1} \epsilon M_{\epsilon}+\frac{(1-m)^{2}}{24}\left(\frac{1+m}{2}\right)^{3} M_{\epsilon}^{-2} \\
& +2 c_{-1} \epsilon M_{\epsilon}+\frac{(1+m)^{2}}{24}\left(\frac{1-m}{2}\right)^{3} M_{\epsilon}^{-2} \\
& +\frac{(1-m)^{2}}{24} \sum_{i=1}^{M_{\epsilon}+1} p\left(p_{i}-p\right)^{2}+\frac{(1+m)^{2}}{24} \sum_{i=1}^{M_{\epsilon}} q\left(q_{i}-q\right)^{2}
\end{aligned}
$$




$$
\begin{aligned}
= & o(\epsilon)+c_{0} \epsilon N_{\epsilon}+\frac{\left(1-m^{2}\right)^{2}}{24 N_{\epsilon}^{2}} \\
& +\frac{(1-m)^{2}}{24} \sum_{i=1}^{M_{\epsilon}+1} p\left(p_{i}-p\right)^{2}+\frac{(1+m)^{2}}{24} \sum_{i=1}^{M_{\epsilon}} q\left(q_{i}-q\right)^{2} .
\end{aligned}
$$

Use $N_{\epsilon}$ for $N$ in Lemma 3.1, and deduce, as in 6.15,

$$
\frac{(1-m)^{2}}{24} \sum_{i=1}^{M_{\epsilon}+1} p\left(p_{i}-p\right)^{2}+\frac{(1+m)^{2}}{24} \sum_{i=1}^{M_{\epsilon}} q\left(q_{i}-q\right)^{2}=o(\epsilon) .
$$

This implies, since $p \sim \epsilon^{1 / 3}$ and $q \sim \epsilon^{1 / 3}$ by 6.14, that $p_{i}-p=o\left(\epsilon^{1 / 3}\right), q_{i}-q=o\left(\epsilon^{1 / 3}\right)$. Therefore $p_{i} \sim \epsilon^{1 / 3}$ and $q_{i} \sim \epsilon^{1 / 3}$.

We turn our attention to the zeros of $v_{\epsilon}^{\prime}$ from the $\alpha$-points of $u_{\epsilon}$.

Proposition 7.2 Let $x_{1}, \ldots, x_{N_{\epsilon}}$ be the $\alpha$-points of $u_{\epsilon}$. Then $v_{\epsilon}^{\prime}$ has exactly $N_{\epsilon}-1$ zeros, denoted by $y_{1}, \ldots, y_{N_{\epsilon}-1}$, in $(0,1)$, distributed between the $\alpha$-points of $u_{\epsilon}$, i.e.

$$
0<x_{1}<y_{1}<x_{2}<y_{2}<\ldots<x_{N_{\epsilon}-1}<y_{N_{\epsilon}-1}<x_{N_{\epsilon}}<1,
$$

with the property $y_{i}=\left(x_{i}+x_{i+1}\right) / 2+o\left(\epsilon^{1 / 3}\right)$. In particular $y_{i+1}-y_{i} \sim \epsilon^{1 / 3}$.

Proof. We first claim that for $i=1, \ldots, M_{\epsilon}$,

$$
v_{\epsilon}^{\prime}\left(x_{2 i-1}\right)=-\frac{(1+m) q_{i}}{2}+o\left(\epsilon^{1 / 3}\right), \quad v_{\epsilon}^{\prime}\left(x_{2 i-2}\right)=\frac{(1-m) p_{i}}{2}+o\left(\epsilon^{1 / 3}\right) .
$$

The careful reader may have noticed that $v_{\epsilon}^{\prime}\left(x_{N_{\epsilon}}\right)$ is not covered here. We will fix this problem later. We assemble a lower bound for $I_{\epsilon}\left(u_{\epsilon}\right)$ one last time, using Lemmas 5.1, 6.3, 7.6) and Proposition 7.1 .

$$
\begin{aligned}
I_{\epsilon}\left(u_{\epsilon}\right) \geqslant & c_{0} \epsilon N_{\epsilon}+\frac{(1-m)^{2}}{24}\left[4 p_{1}^{3}+\sum_{i=2}^{M_{\epsilon}} p_{i}^{3}+4 p_{M_{\epsilon}+1}^{3}\right]+\frac{(1+m)^{2}}{24} \sum_{i=1}^{M_{\epsilon}} q_{i}^{3} \\
& +\sum_{i=2}^{M_{\epsilon}} \frac{p_{i}}{2}\left[v_{\epsilon}^{\prime}\left(x_{2 i-2}\right)-\frac{(1-m) p_{i}}{2}+o\left(\epsilon^{2 / 3}\right)\right]^{2} \\
& +\sum_{i=1}^{M_{\epsilon}} \frac{q_{i}}{2}\left[v_{\epsilon}^{\prime}\left(x_{2 i-1}\right)+\frac{(1+m) q_{i}}{2}+o\left(\epsilon^{2 / 3}\right)\right]^{2}+o(\epsilon) \\
\geqslant & c_{0} \epsilon N_{\epsilon}+\frac{\left(1-m^{2}\right)^{2}}{24 N_{\epsilon}^{2}}+o(\epsilon) \\
& +\sum_{i=2}^{M_{\epsilon}} \frac{p_{i}}{2}\left[v_{\epsilon}^{\prime}\left(x_{2 i-2}\right)-\frac{(1-m) p_{i}}{2}+o\left(\epsilon^{2 / 3}\right)\right]^{2} \\
& +\sum_{i=1}^{M_{\epsilon}} \frac{q_{i}}{2}\left[v_{\epsilon}^{\prime}\left(x_{2 i-1}\right)+\frac{(1+m) q_{i}}{2}+o\left(\epsilon^{2 / 3}\right)\right]^{2}
\end{aligned}
$$


where the last inequality follows from Lemma 5.5 (7.4) as in (7.7). Note that this is the only place where the full power of Lemma 6.3 is realized. We match this lower bound with the upper bound, Lemma $3.1_{l}$, setting $N=N_{\epsilon}$. Then

$\sum_{i=2}^{M_{\epsilon}} \frac{p_{i}}{2}\left[v_{\epsilon}^{\prime}\left(x_{2 i-2}\right)-\frac{(1-m) p_{i}}{2}+o\left(\epsilon^{2 / 3}\right)\right]^{2}+\sum_{i=1}^{M_{\epsilon}} \frac{q_{i}}{2}\left[v_{\epsilon}^{\prime}\left(x_{2 i-1}\right)+\frac{(1+m) q_{i}}{2}+o\left(\epsilon^{2 / 3}\right)\right]^{2}=o(\epsilon)$.

Since $p_{i}, q_{i} \sim \epsilon^{1 / 3}$ (Proposition 7.1, we obtain 7.9.

We now fix the problem about $v_{\epsilon}^{\prime}\left(x_{N_{\epsilon}}\right)$ in this claim. Simply repeat the same argument with 6.19), the other version of Lemma 6.3 mentioned after its proof. Then we find that for $i=$ $1, \ldots, M_{\epsilon}$,

$$
v_{\epsilon}^{\prime}\left(x_{2 i-1}\right)=-\frac{(1-m) p_{i}}{2}+o\left(\epsilon^{1 / 3}\right), \quad v_{\epsilon}^{\prime}\left(x_{2 i}\right)=\frac{(1+m) q_{i}}{2}+o\left(\epsilon^{1 / 3}\right) .
$$

We take up the example of $x_{1}$ and $x_{2}$ between which we will find $y_{1}$. Other cases can be handled similarly. Estimate $v_{\epsilon}^{\prime}\left(x_{1}\right)$ by 7.9 and $v_{\epsilon}^{\prime}\left(x_{2}\right)$ by 7.11 :

$$
v_{\epsilon}^{\prime}\left(x_{1}\right)=-\frac{(1+m) q_{1}}{2}+o\left(\epsilon^{1 / 3}\right), \quad v_{\epsilon}^{\prime}\left(x_{2}\right)=\frac{(1+m) q_{1}}{2}+o\left(\epsilon^{1 / 3}\right)
$$

We make a note here that estimating $v_{\epsilon}^{\prime}\left(x_{2}\right)$ by 7.9$)$ will give $(1+m) q_{1}=(1-m) p_{2}+o\left(\epsilon^{1 / 3}\right)$. By $(7.12)$ there exists $y_{1} \in\left(x_{1}, x_{2}\right)$ such that $v_{\epsilon}^{\prime}\left(y_{1}\right)=0$, since $q_{1} \sim \epsilon^{1 / 3}$ by Proposition 7.1.

Next we estimate $y_{1}-x_{1}$. For this purpose we use 5.1] to find

$$
0=v_{\epsilon}^{\prime}\left(y_{1}\right)=v_{\epsilon}^{\prime}\left(x_{1}\right)+(1+m)\left(y_{1}-x_{1}\right)+O\left(\epsilon^{2 / 3}\right),
$$

which implies, with the help of 7.12,

$$
y_{1}-x_{1}=-\frac{v_{\epsilon}^{\prime}\left(x_{1}\right)}{1+m}+O\left(\epsilon^{2 / 3}\right)=\frac{q_{1}}{2}+o\left(\epsilon^{1 / 3}\right) .
$$

Finally, we see that $y_{1}$, which must be in an $o\left(\epsilon^{1 / 3}\right)$ neighborhood of $\left(x_{1}+x_{2}\right) / 2$, is unique. For by Lemma 6.2 in this neighborhood $v_{\epsilon}^{\prime \prime} \sim 1+m$, so $v_{\epsilon}^{\prime}$ is strictly increasing there.

\section{The one layer local minima of $J_{\epsilon, l}$}

Let $l_{i}=y_{i}-y_{i-1}, i=1, \ldots, N_{\epsilon}$, where $y_{0}=0, y_{N_{\epsilon}}=1$. Between two zero points of $v_{\epsilon}^{\prime}$ we integrate the equation $-v_{\epsilon}^{\prime \prime}=u_{\epsilon}-m$ to find $l_{i}^{-1} \int_{y_{i-1}}^{y_{i}} u_{\epsilon} \mathrm{d} x=m$. This allows us to localize the energy of $u_{\epsilon}$ on $\left(y_{i-1}, y_{i}\right)$. If we set $l_{i} z+y_{i-1}=x, \mathcal{U}_{\epsilon, i}(z)=u_{\epsilon}(x)$, and $\mathcal{V}_{\epsilon, i}(z)=l_{i}^{-2} v_{\epsilon}(x)$, then $\int_{0}^{1} \mathcal{U}_{\epsilon, i} \mathrm{~d} z=m,-\mathcal{V}_{\epsilon, i}^{\prime \prime}=\mathcal{U}_{\epsilon, i}-m, \mathcal{V}_{\epsilon, i}^{\prime}(0)=\mathcal{V}_{\epsilon, i}^{\prime}(1)=0$. More importantly,

$$
\begin{aligned}
I_{\epsilon}\left(u_{\epsilon}\right) & =\sum_{i=1}^{N_{\epsilon}} \int_{y_{i-1}}^{y_{i}}\left[\frac{\epsilon^{2}}{2}\left|u_{\epsilon}^{\prime}\right|^{2}+W\left(u_{\epsilon}\right)+\frac{1}{2}\left|v_{\epsilon}^{\prime}\right|^{2}\right] \mathrm{d} x \\
& =\sum_{i=1}^{N_{\epsilon}} l_{i} \int_{0}^{1}\left[\frac{\epsilon^{2}}{2 l_{i}^{2}}\left|\mathcal{U}_{\epsilon, i}^{\prime}\right|^{2}+W\left(\mathcal{U}_{\epsilon, i}\right)+\frac{l_{i}^{2}}{2}\left|\mathcal{V}_{\epsilon, i}^{\prime}\right|^{2}\right] \mathrm{d} z=\sum_{i=1}^{N_{\epsilon}} l_{i} J_{\epsilon, l_{i}}\left(\mathcal{U}_{\epsilon, i}\right),
\end{aligned}
$$


if we define a new variational functional:

$$
J_{\epsilon, l}(\mathcal{U})=\int_{0}^{1}\left[\frac{\epsilon^{2}}{2 l^{2}}\left|\mathcal{U}^{\prime}\right|^{2}+W(\mathcal{U})+\frac{l^{2}}{2}\left|\left(-D^{2}\right)^{-1 / 2}(\mathcal{U}-m)\right|^{2}\right] \mathrm{d} z, \quad \mathcal{U} \in X_{m} .
$$

This functional has two parameters, $\epsilon$ and $l$. Because of Proposition 7.2, we only need to consider the range of $\epsilon$ and $l$ that satisfies $l \sim \epsilon^{1 / 3}$, i.e. we assume that there exist $C_{1}$ and $C_{2}$ such that

$$
\epsilon \rightarrow 0, \quad C_{1} \epsilon^{1 / 3} \leqslant l \leqslant C_{2} \epsilon^{1 / 3} .
$$

It is sometimes more convenient to use a different pair of parameters, $\varepsilon$ and $d$, where

$$
\varepsilon=\epsilon / l \sim \epsilon^{2 / 3} \rightarrow 0, \quad d=l^{3} / \epsilon \sim 1 .
$$

With respect to these new parameters $J_{\epsilon, l}$ in 8.2 takes the form

$$
J_{\varepsilon, d}(\mathcal{U})=\int_{0}^{1}\left[\frac{\varepsilon^{2}}{2}\left|\mathcal{U}^{\prime}\right|^{2}+W(\mathcal{U})+\frac{\varepsilon d}{2}\left|\left(-D^{2}\right)^{-1 / 2}(\mathcal{U}-m)\right|^{2}\right] \mathrm{d} z .
$$

The Euler-Lagrange equation of this functional is

$$
\left\{\begin{array}{l}
-\varepsilon^{2} \mathcal{U}^{\prime \prime}+f(\mathcal{U})+\varepsilon d\left(-D^{2}\right)^{-1}(\mathcal{U}-m)=\lambda \\
\mathcal{U}^{\prime}(0)=\mathcal{U}^{\prime}(1)=0, \quad \int_{0}^{1} \mathcal{U} \mathrm{d} z=m
\end{array}\right.
$$

It was proved in Theorem 1.1 of [13] that $J_{\varepsilon, d}$ has a number of local minima. We focus on the ones with one transition layer. The theorem asserts that there exists $\delta>0$, independent of $\varepsilon$ and $d$, such that in the ball

$$
B_{\delta}=\left\{\mathcal{U} \in L^{2}(0,1):\left\|\mathcal{U}-\mathcal{U}_{0}\right\|_{2}<\delta\right\}
$$

there is $\mathcal{U}_{\varepsilon}$ with

$$
J_{\varepsilon, d}\left(\mathcal{U}_{\varepsilon}\right)=\inf \left\{J_{\varepsilon, d}(\mathcal{U}): \mathcal{U} \in B_{\delta}\right\},
$$

for all $\varepsilon$ and $d$ in the range (8.4). Here $\mathcal{U}_{0} \in X_{m}$ is the same function as in 3.3 .

Note that in its notation the local minimum $\mathcal{U}_{\varepsilon}$ 's dependence on $d$ is suppressed. Also it was proved in Theorem 1.1 of [13] that

$$
\begin{gathered}
\lim _{\varepsilon \rightarrow 0}\left\|\mathcal{U}_{\varepsilon}-\mathcal{U}_{0}\right\|_{L^{2}(0,1)}=0, \\
\lim _{\varepsilon \rightarrow 0} \varepsilon^{-1} J_{\varepsilon, d}\left(\mathcal{U}_{\varepsilon}\right)=c_{0}+\int_{0}^{1} \frac{d}{2}\left|\left(-D^{2}\right)^{-1 / 2}\left(\mathcal{U}_{0}-m\right)\right|^{2} \mathrm{~d} z .
\end{gathered}
$$

The reversal $\mathcal{U}_{\varepsilon}^{R}$ of $\mathcal{U}_{\varepsilon}$, i.e. $\mathcal{U}_{\varepsilon}^{R}(z)=\mathcal{U}_{\varepsilon}(1-z)$, is a local minimum of $J_{\varepsilon, d}$ in

$$
B_{\delta}^{R}=\left\{\mathcal{U} \in L^{2}(0,1):\left\|\mathcal{U}-\mathcal{U}_{0}^{R}\right\|<\delta\right\},
$$

where $\mathcal{U}_{0}^{R}$ is the reversal of $\mathcal{U}_{0} . \mathcal{U}_{\varepsilon}^{R}$ has properties similar to 8.7 and 8.8 . Here $\delta$ is sufficiently small so that $B_{\delta} \cap B_{\delta}^{R}=\emptyset$. 
Given $\mathcal{U}_{\varepsilon}$ let $\mathcal{V}_{\varepsilon}=\left(-D^{2}\right)^{-1}\left(\mathcal{U}_{\varepsilon}-m\right)$, and $\lambda_{\varepsilon}$ the Lagrange multiplier of 8.6 associated with $\mathcal{U}_{\varepsilon}$. Following the argument of Proposition 4.1, with the help of (8.8), we find

$$
\left\{\begin{array}{l}
\left\|\mathcal{V}_{\varepsilon}\right\|_{L^{\infty}(0,1)}=O(1), \\
\lambda_{\varepsilon}=O\left(\varepsilon^{1 / 2}\right), \\
-1+O\left(\varepsilon^{1 / 2}\right) \leqslant \mathcal{U}_{\varepsilon} \leqslant 1+O\left(\varepsilon^{1 / 2}\right) .
\end{array}\right.
$$

As in the earlier sections, we often study $\mathcal{U}_{\varepsilon}$ on a smaller scale. Let $z_{\varepsilon} \in(0,1)$. Introduce $U_{\varepsilon}(t)=$ $\mathcal{U}_{\varepsilon}\left(\varepsilon t+z_{\varepsilon}\right)$. Then $(8.6)$ and $8.9(2,3)$ imply that $-U_{\varepsilon}^{\prime \prime}+f\left(U_{\varepsilon}\right)=O\left(\varepsilon^{1 / 2}\right)$ and $U_{\varepsilon} \rightarrow G$ locally in $C^{1}$ (at least), where $G$ is a solution of $-G^{\prime \prime}+f(G)=0$. Similarly to Lemma 4.2 we find that $G$ is heteroclinic or \pm 1 . For if this is not true, then $G=\omega$, the local maximum of $W$, or is periodic. In either case, for $\theta>0$,

$$
\liminf _{\varepsilon \rightarrow 0} \varepsilon^{-1} J_{\varepsilon, d}\left(\mathcal{U}_{\varepsilon}\right) \geqslant \liminf _{\varepsilon \rightarrow 0} \varepsilon^{-1} \int_{z_{\varepsilon}-\varepsilon \theta}^{z_{\varepsilon}+\varepsilon \theta} W\left(\mathcal{U}_{\varepsilon}\right) \mathrm{d} z=\int_{-\theta}^{\theta} W(G) \mathrm{d} t .
$$

The last quantity can be made arbitrarily large if we choose $\theta$ large. This contradicts 8.8 .

In this section we do not need to use $\alpha$ to characterize transition layers. But for the sake of consistency we continue to do so. Following the same arguments in Propositions 4.3 4.4 and 4.5, we obtain

PROPOSITION 8.1 1. At every $\alpha$-point $z_{\varepsilon}, \mathcal{U}_{\varepsilon}^{\prime}\left(z_{\varepsilon}\right) \neq 0$.

2. If $z_{\varepsilon}$ is an $\alpha$-point, then $\varepsilon / z_{\varepsilon}=o(1)$ and $\varepsilon /\left(1-z_{\varepsilon}\right)=o(1)$.

3. If $z_{\varepsilon}$ and $z_{\varepsilon}^{*}$ are two $\alpha$-points, then $\varepsilon /\left|z_{\varepsilon}-z_{\varepsilon}^{*}\right|=o(1)$.

Proposition 8.2 When $\varepsilon$ is small, $\mathcal{U}_{\varepsilon}$ has a unique $\alpha$-point, denoted by $z_{\varepsilon}$. As $\varepsilon \searrow 0$,

$$
z_{\varepsilon} \rightarrow \frac{1-m}{2} \text { and }\left\|\mathcal{U}_{\varepsilon}-H\left(\frac{\cdot-z_{\varepsilon}}{\varepsilon}\right)\right\|_{L^{\infty}(0,1)} \rightarrow 0
$$

Proof. To prove the existence of an $\alpha$-point, note that $\int_{0}^{1} \mathcal{U}_{\varepsilon}=m$ implies that there exists $z_{\varepsilon}^{\prime}$ where $\mathcal{U}_{\varepsilon}\left(z_{\varepsilon}^{\prime}\right)=m$. Similarly to the location of $\alpha$-points (Proposition 8.1 ) $), \varepsilon / z_{\varepsilon}^{\prime}=o(1)$ and $\varepsilon /\left(1-z_{\varepsilon}^{\prime}\right)$ $=o(1)$. Moreover $\mathcal{U}_{\varepsilon}\left(\varepsilon t+z_{\varepsilon}^{\prime}\right)$ converges in $C^{1}$ to a heteroclinic solution of $-G^{\prime \prime}+f(G)=0$ with $G(0)=m$ by the remarks following 8.9 . Then $\mathcal{U}_{\varepsilon}\left(z_{\varepsilon}\right)=\alpha$ at a point $z_{\varepsilon}$ such that $\left|z_{\varepsilon}-z_{\varepsilon}^{\prime}\right|=O(\varepsilon)$.

To show the uniqueness of $z_{\varepsilon}$, suppose on the contrary there are two $\alpha$-points, $z_{\varepsilon}$ and $z_{\varepsilon}^{*}$, of $\mathcal{U}_{\varepsilon}$. Without loss of generality assume $\mathcal{U}_{\varepsilon}^{\prime}\left(z_{\varepsilon}\right)>0$ and $\mathcal{U}_{\varepsilon}^{\prime}\left(z_{\varepsilon}^{*}\right)<0$ by Proposition 8.1 . Then by Proposition $8.12,3$ and the remarks after 8.9 , for every $\theta>0$, as $\varepsilon \searrow 0$,

$$
\begin{aligned}
\varepsilon^{-1} \int_{0}^{1}\left[\frac{\varepsilon^{2}}{2}\left|\mathcal{U}_{\varepsilon}^{\prime}\right|^{2}+W\left(\mathcal{U}_{\varepsilon}\right)\right] \mathrm{d} z \\
\geqslant \int_{-\theta}^{\theta}\left[\frac{1}{2}\left|\frac{\mathrm{d}}{\mathrm{d} t} \mathcal{U}_{\varepsilon}\left(\varepsilon t+z_{\varepsilon}\right)\right|^{2}+W\left(\mathcal{U}_{\varepsilon}\left(\varepsilon t+z_{\varepsilon}\right)\right)\right] \mathrm{d} t \\
\quad+\int_{-\theta}^{\theta}\left[\frac{1}{2}\left|\frac{\mathrm{d}}{\mathrm{d} t} \mathcal{U}_{\varepsilon}\left(\varepsilon t+z_{\varepsilon}^{*}\right)\right|^{2}+W\left(\mathcal{U}_{\varepsilon}\left(\varepsilon t+z_{\varepsilon}^{*}\right)\right)\right] \mathrm{d} t \\
\rightarrow \int_{-\theta}^{\theta}\left[\frac{1}{2}\left|H^{\prime}(t)\right|^{2}+W(H(t))\right] \mathrm{d} t+\int_{-\theta}^{\theta}\left[\frac{1}{2}\left|H^{\prime}(-t)\right|^{2}+W(H(-t))\right] \mathrm{d} t \geqslant \frac{3 c_{0}}{2}
\end{aligned}
$$


if we choose $\theta$ large enough. On the other hand,

$$
\int_{0}^{1} \frac{d}{2}\left|\left(-D^{2}\right)^{-1 / 2}\left(\mathcal{U}_{\varepsilon}-m\right)\right|^{2} \mathrm{~d} z \rightarrow \int_{0}^{1} \frac{d}{2}\left|\left(-D^{2}\right)^{-1 / 2}\left(\mathcal{U}_{0}-m\right)\right|^{2} \mathrm{~d} z
$$

because of 8.7 and the continuity of the nonlocal part of $J_{\varepsilon, d}$ in the $L^{2}$ norm. Therefore

$$
\liminf _{\varepsilon \rightarrow 0} \varepsilon^{-1} J_{\varepsilon, d}\left(\mathcal{U}_{\varepsilon}\right) \geqslant \frac{3 c_{0}}{2}+\int_{0}^{1} \frac{d}{2}\left|\left(-D^{2}\right)^{-1 / 2}\left(\mathcal{U}_{0}-m\right)\right|^{2} \mathrm{~d} z
$$

contradicting $(8.8)$.

$\mathcal{U}_{\varepsilon}\left(\varepsilon t+z_{\varepsilon}\right)$ converges locally in $C^{1}$ to $H(t)$ or $H(-t)$. We show that the first case implies the conclusions of this proposition, and the second case does not occur. Assume that $H(t)$ is the local limit. If $\left\|\mathcal{U}_{\varepsilon}-H\left(\frac{\cdot-z_{\varepsilon}}{\varepsilon}\right)\right\|_{\infty}=o(1)$ is false, there exists $h_{\varepsilon} \in(0,1)$ such that $\left|z_{\varepsilon}-h_{\varepsilon}\right| / \varepsilon \rightarrow \infty$ and $\left|\mathcal{U}_{\varepsilon}\left(h_{\varepsilon}\right)-H\left(\left(h_{\varepsilon}-z_{\varepsilon}\right) / \varepsilon\right)\right|$ stays away from 0 . Thus $\left|\mathcal{U}_{\varepsilon}\left(h_{\varepsilon}\right)\right|$ stays away from 1 . Now consider $\mathcal{U}_{\varepsilon}\left(\varepsilon t+h_{\varepsilon}\right)$, which converges locally in $C^{1}$ to a heteroclinic solution of $-G^{\prime \prime}+f(G)=0$. Because the derivative of the heteroclinic solution is never zero and $\mathcal{U}_{\varepsilon}^{\prime}(0)=\mathcal{U}_{\varepsilon}^{\prime}(1)=0, h_{\varepsilon} / \varepsilon \rightarrow \infty$ and $\left(1-h_{\varepsilon}\right) / \varepsilon \rightarrow \infty$. There exists $t_{\varepsilon}=O(1)$ such that $\varepsilon t_{\varepsilon}+h_{\varepsilon} \in(0,1)$ and $\mathcal{U}_{\varepsilon}\left(\varepsilon t_{\varepsilon}+h_{\varepsilon}\right)=\alpha$. But $\left|\varepsilon t_{\varepsilon}+h_{\varepsilon}-z_{\varepsilon}\right| / \varepsilon \rightarrow \infty$. So we have found two $\alpha$-points $z_{\varepsilon}$ and $\varepsilon t_{\varepsilon}+h_{\varepsilon}$, contradicting the uniqueness of $z_{\varepsilon}$. Finally $\left\|\mathcal{U}_{\varepsilon}-H\left(\left(\cdot-z_{\varepsilon}\right) / \varepsilon\right)\right\|_{\infty}=o(1)$ and $\int_{0}^{1} \mathcal{U}_{\varepsilon} \mathrm{d} z=m$ show that $z_{\varepsilon} \rightarrow(1-m) / 2$.

If $\mathcal{U}_{\varepsilon}\left(\varepsilon t+z_{\varepsilon}\right)$ converges locally in $C^{1}$ to $H(-t)$, then the same argument leads to $\| \mathcal{U}_{\varepsilon}-$ $H\left(\left(z_{\varepsilon}-\cdot\right) / \varepsilon\right) \|_{\infty}=o(1)$ and $z_{\varepsilon} \rightarrow(1+m) / 2$. Therefore $\mathcal{U}_{\varepsilon} \in B_{\delta}^{R}$ for small $\varepsilon$, contradicting $B_{\delta} \cap B_{\delta}^{R}=\emptyset$.

We define

$$
\phi_{0}(z)= \begin{cases}-\frac{d\left[\mathcal{V}_{0}(z)-\mathcal{V}_{0}\left(\frac{1-m}{2}\right)\right]}{f^{\prime}(-1)}, & 0<z \leqslant \frac{1-m}{2}, \\ -\frac{d\left[\mathcal{V}_{0}(z)-\mathcal{V}_{0}\left(\frac{1-m}{2}\right)\right]}{f^{\prime}(1)}, & \frac{1-m}{2}<z<1,\end{cases}
$$

where $\mathcal{V}_{0}=\left(-D^{2}\right)^{-1}\left(\mathcal{U}_{0}-m\right)$ (see 3.4 $)$. This function's derivative has a jump discontinuity at $(1-m) / 2$, unless $f^{\prime}(-1)=f^{\prime}(1)$.

\section{PROPOSITION 8.3}

$$
\begin{aligned}
\mathcal{U}_{\varepsilon}(z) & =H\left(\frac{z-z_{\varepsilon}}{\varepsilon}\right)+\phi_{0}(z) \varepsilon+O\left(\varepsilon^{2}\right), \\
z_{\varepsilon} & =\frac{1-m}{2}+c_{3} \varepsilon+O\left(\varepsilon^{2}\right),
\end{aligned}
$$

where $c_{3}=\frac{1}{2}\left(\int_{-\infty}^{0}(H+1) \mathrm{d} t+\int_{0}^{\infty}(H-1) \mathrm{d} t+\int_{0}^{1} \phi_{0} \mathrm{~d} z\right)$.

Proof. The first several steps are similar to those in the proof of Lemma 4.6 Anticipating an asymptotic expansion, we write

$$
\mathcal{U}_{\varepsilon}(z)=H\left(\frac{z-z_{\varepsilon}}{\varepsilon}\right)+\phi_{\varepsilon}(z) \varepsilon .
$$


By $8.91,2), \phi_{\varepsilon} \varepsilon$ satisfies $-\varepsilon^{2}\left(\phi_{\varepsilon} \varepsilon\right)^{\prime \prime}+f^{\prime}(\ldots)\left(\phi_{\varepsilon} \varepsilon\right)=O\left(\varepsilon^{1 / 2}\right)$. Arguing as in Step 2 of the proof of Lemma 4.6 on the intervals $\left(0, z_{\varepsilon}\right)$ and $\left(z_{\varepsilon}, 1\right)$ separately, with the help of Proposition 8.2 which asserts $\phi_{\varepsilon} \varepsilon=o(1)$, we deduce

$$
\phi_{\varepsilon} \varepsilon=O\left(\varepsilon^{1 / 2}\right) \text {. }
$$

Then argue as in Steps 3 and 4 of the same lemma to obtain

$$
\left(\phi_{\varepsilon} \varepsilon\right)^{\prime}=\varepsilon^{-1} O\left(\varepsilon^{1 / 2}\right) \text {. }
$$

Because of (8.11), rewrite the equation for $\phi_{\varepsilon} \varepsilon$ as

$$
-\varepsilon^{2}\left(\phi_{\varepsilon} \varepsilon\right)^{\prime \prime}+f^{\prime}(H)\left(\phi_{\varepsilon} \varepsilon\right)+O(\varepsilon)=\lambda_{\varepsilon} .
$$

Multiply this by $\varepsilon^{-1} H^{\prime}\left(\left(z-z_{\varepsilon}\right) / \varepsilon\right)$ and integrate by parts (as in the proof of Lemma 6.2):

$$
\left[-\varepsilon\left(\phi_{\varepsilon} \varepsilon\right)^{\prime} H^{\prime}\left(\frac{z-z_{\varepsilon}}{\varepsilon}\right)+\left(\phi_{\varepsilon} \varepsilon\right) H^{\prime \prime}\left(\frac{z-z_{\varepsilon}}{\varepsilon}\right)\right]_{0}^{1}=\left[\lambda_{\varepsilon}-O(\varepsilon)\right] \int_{-z_{\varepsilon} / \varepsilon}^{\left(1-z_{\varepsilon}\right) / \varepsilon} H^{\prime}(t) \mathrm{d} t .
$$

The exponential decay rates of $H^{\prime}$ and $H^{\prime \prime}, 8.11$, and 8.12 improve 8.9 ) to

$$
\lambda_{\varepsilon}=O(\varepsilon) \text {. }
$$

This estimate implies that $\phi_{\varepsilon}$ satisfies $-\varepsilon^{2} \phi_{\varepsilon}^{\prime \prime}+f^{\prime}(\ldots) \phi_{\varepsilon}=O(1)$. The argument before 8.11) and (8.12) gives

$$
\phi_{\varepsilon}=O(1), \quad \phi_{\varepsilon}^{\prime}=\varepsilon^{-1} O(1)
$$

improving 8.11 and 8.12 ).

At this point we make a preliminary estimate of $z_{\varepsilon}$. From 8.14 we see that $\mathcal{U}_{\varepsilon}(z)=$ $H\left(\left(z-z_{\varepsilon}\right) / \varepsilon\right)+O(\varepsilon)$. Integrating this over $(0,1)$ yields

$$
\begin{aligned}
m & =\int_{0}^{1} H\left(\frac{z-z_{\varepsilon}}{\varepsilon}\right) \mathrm{d} z+O(\varepsilon)=\varepsilon \int_{-z_{\varepsilon} / \varepsilon}^{\left(1-z_{\varepsilon}\right) / \varepsilon} H(t) \mathrm{d} t+O(\varepsilon) \\
& =\varepsilon\left[\int_{-z_{\varepsilon} / \varepsilon}^{0}(H(t)+1) \mathrm{d} t+\int_{0}^{\left(1-z_{\varepsilon}\right) / \varepsilon}(H(t)-1) \mathrm{d} t+\frac{1-2 z_{\varepsilon}}{\varepsilon}\right]+O(\varepsilon) \\
& =1-2 z_{\varepsilon}+O(\varepsilon) .
\end{aligned}
$$

Therefore

$$
z_{\varepsilon}=\frac{1-m}{2}+O(\varepsilon) .
$$

By (8.14) we write the equation for $\phi_{\varepsilon}$ as

$$
-\varepsilon^{2} \phi_{\varepsilon}^{\prime \prime}+f^{\prime}(H) \phi_{\varepsilon}+O(\varepsilon)+d \mathcal{V}_{\varepsilon}=\frac{\lambda_{\varepsilon}}{\varepsilon} .
$$

Again multiply it by $\varepsilon^{-1} H^{\prime}\left(\left(z-z_{\varepsilon}\right) / \varepsilon\right)$ and integrate by parts:

$$
\begin{aligned}
{\left[-\varepsilon \phi_{\varepsilon}^{\prime} H^{\prime}\left(\frac{z-z_{\varepsilon}}{\varepsilon}\right)+\phi_{\varepsilon} H^{\prime \prime}\left(\frac{z-z_{\varepsilon}}{\varepsilon}\right)\right]_{0}^{1} } & =\int_{0}^{1} \varepsilon^{-1}\left[\frac{\lambda_{\varepsilon}}{\varepsilon}-d \mathcal{V}_{\varepsilon}+O(\varepsilon)\right] H^{\prime}\left(\frac{z-z_{\varepsilon}}{\varepsilon}\right) \mathrm{d} z \\
& =\int_{-z_{\varepsilon} / \varepsilon}^{\left(1-z_{\varepsilon}\right) / \varepsilon}\left[\frac{\lambda_{\varepsilon}}{\varepsilon}-d \mathcal{V}_{\varepsilon}\left(z_{\varepsilon}\right)+O(1) \varepsilon t+O(\varepsilon)\right] H^{\prime}(t) \mathrm{d} t \\
& =2\left[\frac{\lambda_{\varepsilon}}{\varepsilon}-d \mathcal{V}_{\varepsilon}\left(z_{\varepsilon}\right)\right]+O(\varepsilon)
\end{aligned}
$$


We have used the fact $\mathcal{V}_{\varepsilon}^{\prime}=O(1)$, which follows from $\left(8.9\right.$ ) and the regularity theory for $\left(-D^{2}\right)^{-1}$, to reach the second line. The exponential decay rates of $H^{\prime}$ and $H^{\prime \prime}$ in line one imply that

$$
\lambda_{\varepsilon}=\varepsilon d \mathcal{V}_{\varepsilon}\left(z_{\varepsilon}\right)+O\left(\varepsilon^{2}\right),
$$

upgrading 8.13 .

With 8.16 we obtain $-\varepsilon^{2} \phi_{\varepsilon}^{\prime \prime}+f^{\prime}(H) \phi_{\varepsilon}+d\left(\mathcal{V}_{\varepsilon}-\mathcal{V}_{\varepsilon}\left(z_{\varepsilon}\right)\right)=O(\varepsilon)$. On $\left(0, z_{\varepsilon}\right)$ set

$$
\phi_{\varepsilon}(z)=-\frac{d\left(\mathcal{V}_{\varepsilon}(z)-\mathcal{V}_{\varepsilon}\left(z_{\varepsilon}\right)\right)}{f^{\prime}(-1)}+\psi_{\varepsilon} .
$$

Then $\psi_{\varepsilon}$ satisfies

$$
-\varepsilon^{2} \psi_{\varepsilon}^{\prime \prime}+f^{\prime}(H) \psi_{\varepsilon}+\frac{f^{\prime}(-1)-f^{\prime}(H)}{f^{\prime}(-1)} d\left[\mathcal{V}_{\varepsilon}-\mathcal{V}_{\varepsilon}\left(z_{\varepsilon}\right)\right]=O(\varepsilon),
$$

with the boundary conditions $\psi_{\varepsilon}^{\prime}(0)=-\varepsilon^{-2} H^{\prime}\left(-z_{\varepsilon} / \varepsilon\right)=\varepsilon^{-2} O\left(\mathrm{e}^{-C / \varepsilon}\right)$ and $\psi_{\varepsilon}\left(z_{\varepsilon}\right)=0$. Note that because of $\mathcal{V}_{\varepsilon}^{\prime \prime}=O(1)$ by $(8.9 \beta)$, and the exponential convergence rate of $H$ to -1 at $-\infty$,

$$
\left|\left[f^{\prime}(-1)-f^{\prime}\left(H\left(\frac{z-z_{\varepsilon}}{\varepsilon}\right)\right)\right]\left(\mathcal{V}_{\varepsilon}(z)-\mathcal{V}_{\varepsilon}\left(z_{\varepsilon}\right)\right)\right| \leqslant C|(H(t)+1) \varepsilon t| \leqslant C \varepsilon .
$$

So the equation for $\psi_{\varepsilon}$ is further simplified to $-\varepsilon^{2} \psi_{\varepsilon}^{\prime \prime}+f^{\prime}(H) \psi_{\varepsilon}=O(\varepsilon)$. Then argue as in 8.11 to conclude that $\psi_{\varepsilon}=O(\varepsilon)$.

In summary we have shown, after a similar argument on $\left(z_{\varepsilon}, 1\right)$,

$$
\mathcal{U}_{\varepsilon}(z)= \begin{cases}H\left(\frac{z-z_{\varepsilon}}{\varepsilon}\right)-\frac{\varepsilon d\left(\mathcal{V}_{\varepsilon}(z)-\mathcal{V}_{\varepsilon}\left(z_{\varepsilon}\right)\right)}{f^{\prime}(-1)}+O\left(\varepsilon^{2}\right), & z \in\left(0, z_{\varepsilon}\right), \\ \alpha, & z=z_{\varepsilon}, \\ H\left(\frac{z-z_{\varepsilon}}{\varepsilon}\right)-\frac{\varepsilon d\left(\mathcal{V}_{\varepsilon}(z)-\mathcal{V}_{\varepsilon}\left(z_{\varepsilon}\right)\right)}{f^{\prime}(1)}+O\left(\varepsilon^{2}\right), & z \in\left(z_{\varepsilon}, 1\right) .\end{cases}
$$

To complete the proof of the first estimate of this proposition, we compare $\mathcal{V}_{\varepsilon}$ to $\mathcal{V}_{0}$. Let $\mathcal{Z}=$ $\mathcal{V}_{\varepsilon}-\mathcal{V}_{0}=\left(-D^{2}\right)^{-1}\left(\mathcal{U}_{\varepsilon}-\mathcal{U}_{0}\right)$. According to 8.14 and 8.15 ,

$$
\begin{aligned}
\left\|\mathcal{U}_{\varepsilon}-\mathcal{U}_{0}\right\|_{1} & =\int_{0}^{1}\left|H\left(\frac{z-z_{\varepsilon}}{\varepsilon}\right)-\mathcal{U}_{0}\right| \mathrm{d} z+O(\varepsilon) \\
& =\varepsilon \int_{-(1-m) /(2 \varepsilon)}^{(1+m) /(2 \varepsilon)}\left|H(t+O(1))-\mathcal{U}_{0}\left(\varepsilon t+\frac{1-m}{2}\right)\right| \mathrm{d} t+O(\varepsilon)=O(\varepsilon) .
\end{aligned}
$$

Integrating the linear differential equation for $\mathcal{Z}$ we see that

$$
\mathcal{V}_{\varepsilon}=\mathcal{V}_{0}+O(\varepsilon), \quad \mathcal{V}_{\varepsilon}^{\prime}=\mathcal{V}_{0}^{\prime}+O(\varepsilon)
$$

The first estimate of the proposition then follows.

To establish the second estimate, integrate the first estimate over $(0,1)$ :

$$
\begin{aligned}
m+O\left(\varepsilon^{2}\right) & =\int_{0}^{1} H\left(\frac{z-z_{\varepsilon}}{\varepsilon}\right) \mathrm{d} z+\varepsilon \int_{0}^{1} \phi_{0} \mathrm{~d} z \\
& =\varepsilon\left[\int_{-z_{\varepsilon} / \varepsilon}^{0}(H(t)+1) \mathrm{d} t+\int_{0}^{\left(1-z_{\varepsilon}\right) / \varepsilon}(H(t)-1) \mathrm{d} t+\frac{1-2 z_{\varepsilon}}{\varepsilon}+\int_{0}^{1} \phi_{0} \mathrm{~d} z\right] \\
& =1-2 z_{\varepsilon}+\varepsilon\left[\int_{\infty}^{0}(H+1) \mathrm{d} t+\int_{0}^{\infty}(H-1) \mathrm{d} t+\int_{0}^{1} \phi_{0} \mathrm{~d} z+O\left(\mathrm{e}^{-C / \varepsilon}\right)\right] .
\end{aligned}
$$


The next result will be very handy later.

LEMMA 8.4 Let $F \in C^{2}(-\infty, \infty)$ be such that $F( \pm 1)=0$. Then

$$
\int_{0}^{1} F\left(\mathcal{U}_{\varepsilon}\right) \mathrm{d} z=\varepsilon \int_{-\infty}^{\infty} F(H) \mathrm{d} t+\varepsilon \int_{0}^{1} F^{\prime}( \pm 1) \phi_{0} \mathrm{~d} z+O\left(\varepsilon^{2}\right),
$$

where \pm 1 is -1 on $(0,(1-m) / 2)$ and 1 on $((1-m) / 2,1)$.

Proof. According to Proposition 8.3

$$
\begin{aligned}
\int_{0}^{1} F\left(\mathcal{U}_{\varepsilon}\right) \mathrm{d} z & =\int_{0}^{1} F\left(H\left(\frac{z-z_{\varepsilon}}{\varepsilon}\right)+\varepsilon \phi_{0}+O\left(\varepsilon^{2}\right)\right) \mathrm{d} z \\
& =\varepsilon \int_{-z_{\varepsilon} / \varepsilon}^{\left(1-z_{\varepsilon}\right) / \varepsilon} F(H(t)) \mathrm{d} t+\varepsilon \int_{0}^{1} F^{\prime}\left(H\left(\frac{z-z_{\varepsilon}}{\varepsilon}\right)\right) \phi_{0} \mathrm{~d} z+O\left(\varepsilon^{2}\right) \\
& =\varepsilon \int_{-\infty}^{\infty} F(H) \mathrm{d} t+\varepsilon \int_{0}^{1} F^{\prime}( \pm 1) \phi_{0} \mathrm{~d} z+O\left(\varepsilon^{2}\right)
\end{aligned}
$$

\section{The second variation of $J_{\varepsilon, d}$}

We now study the second variation of $J_{\varepsilon}$ at $\mathcal{U}_{\varepsilon}$ and give a bound on the principal eigenvalue of the linearized operator of 8.6 .

Proposition 9.1 There is $c_{4}>0$ such that for all $\varphi \in W^{1,2}(0,1)$ with $\operatorname{Ave}(\varphi)=0$,

$$
\int_{0}^{1}\left[\varepsilon^{2}\left|\varphi^{\prime}\right|^{2}+f^{\prime}\left(\mathcal{U}_{\varepsilon}\right) \varphi^{2}+\varepsilon d\left|\left(-D^{2}\right)^{-1 / 2} \varphi\right|^{2}\right] \mathrm{d} z \geqslant c_{4} \varepsilon \int_{0}^{1} \varphi^{2} \mathrm{~d} z
$$

Proof. All we need to prove is that if $\Lambda$ is an eigenvalue of the eigenvalue problem

$$
\left\{\begin{array}{l}
-\varepsilon^{2} \psi^{\prime \prime}+f^{\prime}\left(\mathcal{U}_{\varepsilon}\right) \psi+\varepsilon d\left(-D^{2}\right)^{-1} \psi=\eta+\Lambda \psi, \\
\psi^{\prime}(0)=\psi^{\prime}(1)=0, \quad \operatorname{Ave}(\psi)=0, \quad \psi \neq \equiv 0
\end{array}\right.
$$

then $\Lambda \geqslant c_{4} \varepsilon$ for some constant $c_{4}>0$. Since $\mathcal{U}_{\varepsilon}$ minimizes $J_{\varepsilon}$ locally, $\Lambda$ must be $\geqslant 0$. Suppose the assertion of the proposition is false. Then $\Lambda=o(\varepsilon)$.

We normalize the eigenfunction $\psi$ so that $\|\psi\|_{2}=1$. Let $H_{\varepsilon}$ be a modification of $H$ so that $H_{\varepsilon}(t)=-1$ if $t \leqslant-z_{\varepsilon} /(2 \varepsilon)$ and $H_{\varepsilon}(t)=1$ if $t \geqslant\left(1-z_{\varepsilon}\right) /(2 \varepsilon)$. Moreover $H_{\varepsilon}=H+O\left(\mathrm{e}^{-C / \varepsilon}\right)$, $H_{\varepsilon}^{\prime}=H^{\prime}+O\left(\mathrm{e}^{-C / \varepsilon}\right)$, and $H_{\varepsilon}^{\prime \prime}=H^{\prime \prime}+O\left(\mathrm{e}^{-C / \varepsilon}\right)$. Then let $h_{\varepsilon}(z)=\varepsilon^{-1} H_{\varepsilon}^{\prime}\left(\left(z-z_{\varepsilon}\right) / \varepsilon\right)$. This $h_{\varepsilon}$ has compact support. It follows from Lemma 2.2 that for all $\varphi \in W^{1,2}(0,1)$ with $\int_{0}^{1} \varphi h_{\varepsilon}=0$,

$$
\int_{0}^{1}\left[\varepsilon^{2}\left|\varphi^{\prime}\right|^{2}+f^{\prime}(H) \varphi^{2}\right] \mathrm{d} z \geqslant c_{5} \int_{0}^{1} \varphi^{2} \mathrm{~d} z
$$

We decompose $\psi=c h_{\varepsilon}+\psi^{\perp}$ with $\int_{0}^{1} h_{\varepsilon} \psi^{\perp} \mathrm{d} z=0$. Let $A=\left(-D^{2}\right)^{-1}\left(h_{\varepsilon}-\operatorname{Ave}\left(h_{\varepsilon}\right)\right)$ and $B=\left(-D^{2}\right)^{-1}\left(\psi^{\perp}-\operatorname{Ave}\left(\psi^{\perp}\right)\right)$. Note that

$$
A=O(1), \quad B=\left\|\psi^{\perp}\right\|_{2} O(1),
$$


and $\left(-D^{2}\right)^{-1} \psi=c A+B$. By integrating the decomposition of $\psi$ we find

$$
\int_{0}^{1} \psi^{\perp}=-2 c
$$

By integrating (9.1) we observe

$$
\eta=\int_{0}^{1} f^{\prime}\left(\mathcal{U}_{\varepsilon}\right) \psi \mathrm{d} z=\int_{0}^{1}\left[f^{\prime}(H)+O(\varepsilon)\right]\left(c h_{\varepsilon}+\psi^{\perp}\right) \mathrm{d} z
$$

After estimating the right side, we deduce

$$
|\eta|=|c| O(\varepsilon)+\left\|\psi^{\perp}\right\|_{2} O(1) .
$$

The equation for $\psi^{\perp}$ is

$-\varepsilon^{2}\left(\psi^{\perp}\right)^{\prime \prime}+f^{\prime}\left(\mathcal{U}_{\varepsilon}\right) \psi^{\perp}+\varepsilon d(c A+B)+c\left[f^{\prime}\left(\mathcal{U}_{\varepsilon}\right)-f^{\prime}(H)\right] h_{\varepsilon}+O\left(\mathrm{e}^{-C / \varepsilon}\right)=\eta+\Lambda\left(c h_{\varepsilon}+\psi^{\perp}\right)$.

Multiply this by $\psi^{\perp}$ and integrate by parts to obtain

$$
\begin{aligned}
\int_{0}^{1}\left[\varepsilon^{2}\left|\left(\psi^{\perp}\right)^{\prime}\right|^{2}\right. & \left.+f^{\prime}\left(\mathcal{U}_{\varepsilon}\right)\left|\psi^{\perp}\right|^{2}+\varepsilon d\left|B^{\prime}\right|^{2}-\Lambda\left|\psi^{\perp}\right|^{2}\right] \mathrm{d} z \\
& =\int_{0}^{1}\left[-c\left(f^{\prime}\left(\mathcal{U}_{\varepsilon}\right)-f^{\prime}(H)\right) h_{\varepsilon} \psi^{\perp}-c \varepsilon d A \psi^{\perp}+\eta \psi^{\perp}+\psi^{\perp} O\left(\mathrm{e}^{-C / \varepsilon}\right)\right] \mathrm{d} z
\end{aligned}
$$

By 9.2 and the assumption on $\Lambda$ we find that the first line is $\geqslant c_{6} \int_{0}^{1}\left|\psi^{\perp}\right|^{2} \mathrm{~d} z$. To estimate the second line we note, with the help of 9.3,

$$
\begin{aligned}
\left|\int_{0}^{1}-c\left(f^{\prime}\left(\mathcal{U}_{\varepsilon}\right)-f^{\prime}(H)\right) h_{\varepsilon} \psi^{\perp} \mathrm{d} z\right| & =|c| \cdot\left\|\psi^{\perp}\right\|_{2} O\left(\varepsilon^{3 / 2}\right), \\
\left|\int_{0}^{1}-c \varepsilon d A \psi^{\perp} \mathrm{d} z\right| & =|c| \cdot\left\|\psi^{\perp}\right\|_{2} O(\varepsilon), \\
\left|\int_{0}^{1} \eta \psi^{\perp} \mathrm{d} z\right| & =2|\eta| \cdot|c|, \\
\left|\int_{0}^{1} O\left(\mathrm{e}^{-C / \varepsilon}\right) \psi^{\perp} \mathrm{d} z\right| & =\left\|\psi^{\perp}\right\|_{2} O\left(\mathrm{e}^{-C / \varepsilon}\right) .
\end{aligned}
$$

The first one here is less obvious. Note that

$$
\begin{aligned}
\left\|\left(f^{\prime}\left(\mathcal{U}_{\varepsilon}\right)-f^{\prime}(H)\right) h_{\varepsilon}\right\|_{2}^{2} & \leqslant C \int_{0}^{1}\left(\varepsilon \phi_{0}+O\left(\varepsilon^{2}\right)\right)^{2} h_{\varepsilon}^{2} \mathrm{~d} z \\
& =C \varepsilon^{3} \int_{-z_{\varepsilon} / \varepsilon}^{\left(1-z_{\varepsilon}\right) / \varepsilon}\left[\frac{\phi_{0}\left(\varepsilon t+z_{\varepsilon}\right)-\phi_{0}((1-m) / 2)}{\varepsilon}+O(1)\right]^{2} H_{\varepsilon}^{\prime}(t)^{2} \mathrm{~d} t \\
& \leqslant C \varepsilon^{3} \int_{-z_{\varepsilon} / \varepsilon}^{\left(1-z_{\varepsilon}\right) / \varepsilon}(|t|+O(1))^{2} H_{\varepsilon}^{\prime}(t)^{2} \mathrm{~d} t=O\left(\varepsilon^{3}\right)
\end{aligned}
$$

by Proposition 8.3, $\phi_{0}((1-m) / 2)=0$ and the Lipschitz continuity of $\phi_{0}$. 
Therefore the last integral identity implies

$$
c_{6}\left\|\psi^{\perp}\right\|_{2}^{2} \leqslant|c| \cdot\left\|\psi^{\perp}\right\|_{2} O(\varepsilon)+2|\eta| \cdot|c|+\left\|\psi^{\perp}\right\|_{2} O\left(\mathrm{e}^{-C / \varepsilon}\right),
$$

which, combined with 9.5 , leads to

$$
\begin{aligned}
\left\|\psi^{\perp}\right\|_{2} & \leqslant|c| O(1)+O\left(\mathrm{e}^{-C / \varepsilon}\right), \\
|\eta| & \leqslant|c| O(1)+O\left(\mathrm{e}^{-C / \varepsilon}\right) .
\end{aligned}
$$

It also leads, with the help of $2|c|=\left|\int_{0}^{1} \psi^{\perp} \mathrm{d} z\right| \leqslant\left\|\psi^{\perp}\right\|_{2}$, to

$$
|c| \leqslant|\eta| O(1)+O\left(\mathrm{e}^{-C / \varepsilon}\right) .
$$

Next we multiply the equation for $\psi^{\perp}$ by $h_{\varepsilon}$ and integrate by parts to obtain

$$
\int_{0}^{1}\left[\psi^{\perp} O\left(\mathrm{e}^{-C / \varepsilon}\right)+\varepsilon d(c A+B) h_{\varepsilon}+\left(f^{\prime}\left(\mathcal{U}_{\varepsilon}\right)-f^{\prime}(H)\right) h_{\varepsilon} \psi\right] \mathrm{d} z=\int_{0}^{1}\left(\eta h_{\varepsilon}+\Lambda c h_{\varepsilon}^{2}\right) \mathrm{d} z .
$$

We estimate each term, using 9.7):

$$
\begin{aligned}
\left|\int_{0}^{1} \psi^{\perp} O\left(\mathrm{e}^{-C / \varepsilon}\right) \mathrm{d} z\right| & =|c| O\left(\mathrm{e}^{-C / \varepsilon}\right)+O\left(\mathrm{e}^{-C / \varepsilon}\right), \\
\left|\int_{0}^{1} \varepsilon d c A h_{\varepsilon} \mathrm{d} z\right| & =|c| O(\varepsilon) \\
\left|\int_{0}^{1} \varepsilon d B h_{\varepsilon} \mathrm{d} z\right| & =|c| O(\varepsilon)+O\left(\mathrm{e}^{-C / \varepsilon}\right) \\
\left|\int_{0}^{1}\left(f^{\prime}\left(\mathcal{U}_{\varepsilon}\right)-f^{\prime}(H)\right) h_{\varepsilon} \psi \mathrm{d} z\right| & =|c| O(\varepsilon)+O\left(\mathrm{e}^{-C / \varepsilon}\right) \\
\int_{0}^{1} \eta h_{\varepsilon} \mathrm{d} z & =-2 \eta \\
\int_{0}^{1} \Lambda c h_{\varepsilon}^{2} \mathrm{~d} z & =|c| O(1)
\end{aligned}
$$

All of the above are easy with the possible exception of the fourth estimate. One writes $\psi=c h_{\varepsilon}+$ $\psi^{\perp}$, so

$$
\left|\int_{0}^{1}\left(f^{\prime}\left(\mathcal{U}_{\varepsilon}\right)-f^{\prime}(H)\right) h_{\varepsilon} \psi^{\perp} \mathrm{d} z\right| \leqslant\left\|\left(f^{\prime}\left(\mathcal{U}_{\varepsilon}\right)-f^{\prime}(H)\right) h_{\varepsilon}\right\|_{2}\left\|\psi^{\perp}\right\|_{2}=|c| O\left(\varepsilon^{3 / 2}\right)+O\left(\mathrm{e}^{-C / \varepsilon}\right),
$$

by 9.6 . And arguing as in 9.6 we find

$$
\left|\int_{0}^{1}\left(f^{\prime}\left(\mathcal{U}_{\varepsilon}\right)-f^{\prime}(H)\right) h_{\varepsilon}^{2} \mathrm{~d} z\right|=O(\varepsilon) .
$$

The last integral identity then implies $\eta=|c| o(1)+O\left(\mathrm{e}^{-C / \varepsilon}\right)$. Because of $9.7,, 9.8$ and 9.9 we have $|c|=O\left(\mathrm{e}^{-C / \varepsilon}\right)$ and $\left\|\psi^{\perp}\right\|_{2}=O\left(\mathrm{e}^{-C / \varepsilon}\right)$. So $\|\psi\|_{2}=O\left(\mathrm{e}^{-C / \varepsilon}\right)$, contradicting $\|\psi\|_{2}=1$. 
The related result in [5] has no $\varepsilon$ after $c_{4}$. The reason is that the extra condition that $\varphi(1 / 2)$ $=0$ was assumed. Here without this condition we have small eigenvalues. A consequence of this proposition is that $\mathcal{U}_{\varepsilon}$ is unique.

PRoposition 9.2 For small $\varepsilon$, if $\mathcal{U}_{\varepsilon}, \mathcal{U}_{\varepsilon}^{*} \in B_{\delta}$ satisfy $J_{\varepsilon, d}\left(\mathcal{U}_{\varepsilon}\right)=J_{\varepsilon, d}\left(\mathcal{U}_{\varepsilon}^{*}\right)=\inf \left\{J_{\varepsilon, d}(\mathcal{U})\right.$ : $\left.\mathcal{U} \in B_{\delta}\right\}$, then $\mathcal{U}_{\varepsilon}=\mathcal{U}_{\varepsilon}^{*}$. The same is also true in $B_{\delta}^{R}$.

Proof. Let $\mathcal{U}_{\varepsilon}$ and $\mathcal{U}_{\varepsilon}^{*}$ be as in the statement. We first show that $\mathcal{U}_{\varepsilon}-\mathcal{U}_{\varepsilon}^{*}=O\left(\varepsilon^{2}\right)$, and then use Proposition 9.1 to conclude that they are identical.

The first estimate of Proposition 8.3 asserts that

$$
\mathcal{U}_{\varepsilon}^{*}-\mathcal{U}_{\varepsilon}=H\left(\frac{z-z_{\varepsilon}^{*}}{\varepsilon}\right)-H\left(\frac{z-z_{\varepsilon}}{\varepsilon}\right)+O\left(\varepsilon^{2}\right),
$$

where $z_{\varepsilon}\left(z_{\varepsilon}^{*}\right.$ respectively) is the $\alpha$-point of $\mathcal{U}_{\varepsilon}$ ( $\mathcal{U}_{\varepsilon}^{*}$ respectively). The second estimate of Proposition 8.3 says $z_{\varepsilon}^{*}-z_{\varepsilon}=O\left(\varepsilon^{2}\right)$. Therefore

$$
\mathcal{U}_{\varepsilon}^{*}-\mathcal{U}_{\varepsilon}=\frac{z_{\varepsilon}-z_{\varepsilon}^{*}}{\varepsilon} H^{\prime}\left(\frac{z-z_{\varepsilon}}{\varepsilon}\right)+O\left(\varepsilon^{2}\right)=\kappa(\varepsilon) H^{\prime}\left(\frac{z-z_{\varepsilon}}{\varepsilon}\right) \varepsilon+O\left(\varepsilon^{2}\right),
$$

where $\kappa(\varepsilon)=\left(z_{\varepsilon}-z_{\varepsilon}^{*}\right) / \varepsilon^{2}=O(1)$.

We next show that $\kappa(\varepsilon)=O\left(\varepsilon^{1 / 2}\right)$. Let $\mathcal{W}_{\varepsilon}=\mathcal{U}_{\varepsilon}^{*}-\mathcal{U}_{\varepsilon}, \mathcal{Z}_{\varepsilon}=\mathcal{V}_{\varepsilon}^{*}-\mathcal{V}_{\varepsilon}$, where $\mathcal{V}_{\varepsilon}=$ $\left(-D^{2}\right)^{-1}\left(\mathcal{U}_{\varepsilon}-m\right)$ and $\mathcal{V}_{\varepsilon}^{*}=\left(-D^{2}\right)^{-1}\left(\mathcal{U}_{\varepsilon}^{*}-m\right)$. Then $\mathcal{W}_{\varepsilon}$ satisfies

$$
-\varepsilon^{2} \mathcal{W}_{\varepsilon}^{\prime \prime}+f^{\prime}\left(\mathcal{U}_{\varepsilon}\right) \mathcal{W}_{\varepsilon}+\varepsilon d \mathcal{Z}_{\varepsilon}+\left[f\left(\mathcal{U}_{\varepsilon}^{*}\right)-f\left(\mathcal{U}_{\varepsilon}\right)-f^{\prime}\left(\mathcal{U}_{\varepsilon}\right) \mathcal{W}_{\varepsilon}\right]=\lambda_{\varepsilon}^{*}-\lambda_{\varepsilon}
$$

Multiply by $\mathcal{W}_{\varepsilon}$ and integrate by parts to deduce, with the help of 9.10:

$$
\begin{aligned}
\int_{0}^{1}\left[\varepsilon^{2}\left|\mathcal{W}_{\varepsilon}^{\prime}\right|^{2}\right. & \left.+f^{\prime}\left(\mathcal{U}_{\varepsilon}\right) \mathcal{W}_{\varepsilon}^{2}+\varepsilon d\left|\mathcal{Z}_{\varepsilon}^{\prime}\right|^{2}\right] \mathrm{d} z \\
& =-\int_{0}^{1}\left[f\left(\mathcal{U}_{\varepsilon}^{*}\right)-f\left(\mathcal{U}_{\varepsilon}\right)-f^{\prime}\left(\mathcal{U}_{\varepsilon}\right) \mathcal{W}_{\varepsilon}\right] \mathcal{W}_{\varepsilon} \mathrm{d} z \\
& =-\frac{1}{2} \int_{0}^{1} f^{\prime \prime}\left(H\left(\frac{z-z_{\varepsilon}}{\varepsilon}\right)+O(\varepsilon)\right)\left(\kappa(\varepsilon) H^{\prime}\left(\frac{z-z_{\varepsilon}}{\varepsilon}\right) \varepsilon+O\left(\varepsilon^{2}\right)\right)^{3} \mathrm{~d} z \\
& =-\frac{\varepsilon^{4}}{2} \int_{-z_{\varepsilon} / \varepsilon}^{\left(1-z_{\varepsilon}\right) / \varepsilon} f^{\prime \prime}(H(t)+O(\varepsilon))\left(\kappa(\varepsilon) H^{\prime}(t)+O(\varepsilon)\right)^{3} \mathrm{~d} t \\
& =-\frac{\kappa(\varepsilon) \varepsilon^{4}}{2} \int_{-\infty}^{\infty} f^{\prime \prime}(H)\left(H^{\prime}\right)^{3} \mathrm{~d} t+O\left(\varepsilon^{5}\right)=O\left(\varepsilon^{5}\right),
\end{aligned}
$$

since by [2.4] and integration by parts

$$
\int_{-\infty}^{\infty} f^{\prime \prime}(H)\left(H^{\prime}\right)^{3} \mathrm{~d} t=\int_{-1}^{1} 2 f^{\prime \prime}(H) W(H) \mathrm{d} H=-\left.(f(H))^{2}\right|_{H=-1} ^{H=1}=0 .
$$

Note that when the Taylor expansion is used in line three of 9.11, by Proposition 8.3 and 9.10 , both $\mathcal{U}_{\varepsilon}$ and $\mathcal{U}_{\varepsilon}^{*}$ are $H\left(\left(z-z_{\varepsilon}\right) / \varepsilon\right)+O(\varepsilon)$. So we put it in $f^{\prime \prime}(\ldots)$. 
Combining 9.11 with Proposition 9.1 we obtain $\int_{0}^{1}\left|\mathcal{W}_{\varepsilon}\right|^{2} \mathrm{~d} z=O\left(\varepsilon^{4}\right)$. But on the other hand (9.10) implies

$$
\begin{aligned}
\int_{0}^{1}\left|\mathcal{W}_{\varepsilon}\right|^{2} \mathrm{~d} z & =\int_{0}^{1}\left|\kappa(\varepsilon) H^{\prime}\left(\frac{z-z_{\varepsilon}}{\varepsilon}\right) \varepsilon+O\left(\varepsilon^{2}\right)\right|^{2} \mathrm{~d} z=\varepsilon^{3} \int_{-z_{\varepsilon} / \varepsilon}^{\left(1-z_{\varepsilon}\right) / \varepsilon}\left|\kappa(\varepsilon) H^{\prime}(t)+O(\varepsilon)\right|^{2} \mathrm{~d} t \\
& =\kappa^{2}(\varepsilon) \varepsilon^{3} \int_{-\infty}^{\infty}\left|H^{\prime}\right|^{2} \mathrm{~d} t+O\left(\varepsilon^{4}\right) .
\end{aligned}
$$

Therefore $\kappa(\varepsilon)=O\left(\varepsilon^{1 / 2}\right)$. And hence $\mathcal{W}_{\varepsilon}=O\left(\varepsilon^{3 / 2}\right)$ by 9.10 .

Finally, we revisit the first two lines of 9.11 , which imply

$$
\begin{aligned}
\int_{0}^{1}\left[\varepsilon^{2}\left|\mathcal{W}_{\varepsilon}^{\prime}\right|^{2}+f^{\prime}\left(\mathcal{U}_{\varepsilon}\right) \mathcal{W}_{\varepsilon}^{2}+\varepsilon d\left|\mathcal{Z}_{\varepsilon}^{\prime}\right|^{2}\right] \mathrm{d} z & \leqslant C \int_{0}^{1}\left|\mathcal{W}_{\varepsilon}\right|^{3} \mathrm{~d} z \\
& \leqslant C\left\|\mathcal{W}_{\varepsilon}\right\|_{L^{\infty}(0,1)} \int_{0}^{1} \mathcal{W}_{\varepsilon}^{2} \mathrm{~d} z \leqslant C \varepsilon^{3 / 2} \int_{0}^{1} \mathcal{W}_{\varepsilon}^{2} \mathrm{~d} z .
\end{aligned}
$$

Proposition 9.1 yields $c_{4} \varepsilon \int_{0}^{1} \mathcal{W}_{\varepsilon}^{2} \mathrm{~d} z \leqslant C \varepsilon^{3 / 2} \int_{0}^{1} \mathcal{W}_{\varepsilon}^{2} \mathrm{~d} z$. Thus $\mathcal{W}_{\varepsilon}=0$.

We return to the parameters $\epsilon$ and $l$. Rename $\mathcal{U}_{\varepsilon}$, the unique minimum in $B_{\delta}, \mathcal{U}_{\epsilon, l}$. The nondegeneracy implied by Proposition 9.1 allows us to apply the implicit function theorem to conclude that $\mathcal{U}_{\epsilon, l}$ is differentiable in $l$ under the $W^{1,2}$ norm. Let $\mathcal{W}_{\epsilon, l}=\partial \mathcal{U}_{\epsilon, l} / \partial l$.

\section{PROPOSITION 9.3}

$$
\mathcal{W}_{\epsilon, l}=H^{\prime}\left(\frac{l\left(z-z_{\varepsilon}\right)}{\epsilon}\right) \frac{z-z_{\varepsilon}}{\epsilon}-\operatorname{Ave}\left(H^{\prime}\left(\frac{l\left(z-z_{\varepsilon}\right)}{\epsilon}\right) \frac{z-z_{\varepsilon}}{\epsilon}\right)+\phi,
$$

with $\|\phi\|_{2}=O(1 / l)$. And

$$
\phi=c H^{\prime}\left(\frac{l\left(z-z_{\varepsilon}\right)}{\epsilon}\right) \frac{l}{\epsilon}+\phi^{\perp}
$$

with $\int_{0}^{1} H^{\prime}\left(l\left(z-z_{\varepsilon}\right) / \epsilon\right) \phi^{\perp} \mathrm{d} z=0, c=O(1)$ and $\left\|\phi^{\perp}\right\|_{2}=O(l)$.

Proof. Differentiate 8.6 with respect to $l$ to obtain the equation

$$
-\left(\frac{\epsilon}{l}\right)^{2} \mathcal{W}_{\epsilon, l}^{\prime \prime}+f^{\prime}\left(\mathcal{U}_{\epsilon, l}\right) \mathcal{W}_{\epsilon, l}+l^{2}\left(-D^{2}\right)^{-1} \mathcal{W}_{\epsilon, l}+\frac{2}{l} f\left(\mathcal{U}_{\epsilon, l}\right)+4 l \mathcal{V}_{\epsilon, l}-\frac{2 \lambda_{\varepsilon}}{l}=\lambda_{l}
$$

for $\mathcal{W}_{\epsilon, l}$, where $\lambda_{l}$ is the derivative of $\lambda_{\varepsilon}$ with respect to $l$.

As in the proof of Proposition 9.1 we replace $H$ by $H_{\varepsilon}$. Define $g_{\varepsilon}=H_{\varepsilon}^{\prime}\left(l\left(z-z_{\varepsilon}\right) / \epsilon\right)\left(z-z_{\varepsilon}\right) / \epsilon$, and $\varphi=\mathcal{W}_{\epsilon, l}-\left(g_{\varepsilon}-\operatorname{Ave}\left(g_{\varepsilon}\right)\right)$. Then $g_{\varepsilon}$ satisfies the equation

$$
-\left(\frac{\epsilon}{l}\right)^{2} g_{\varepsilon}^{\prime \prime}+f^{\prime}(H) g_{\varepsilon}+\frac{2}{l} f(H)=O\left(\mathrm{e}^{-C / \varepsilon}\right) .
$$

Subtract this from 9.12) and use the fact $\operatorname{Ave}\left(g_{\varepsilon}\right)=O(l)$ to deduce the equation for $\varphi$ :

$$
-(\epsilon / l)^{2} \varphi^{\prime \prime}+f^{\prime}\left(\mathcal{U}_{\epsilon, l}\right) \varphi+l^{2}\left(-D^{2}\right)^{-1} \mathcal{W}_{\epsilon, l}+O(l)=\lambda_{l} .
$$


After defining $A=\left(-D^{2}\right)^{-1}\left(g_{\varepsilon}-\operatorname{Ave}\left(g_{\varepsilon}\right)\right)$ and $B=\left(-D^{2}\right)^{-1}(\varphi-\operatorname{Ave}(\varphi))$, we multiply the above equation by $\varphi$ and integrate by parts:

$$
\int_{0}^{1}\left[(\epsilon / l)^{2}\left|\varphi^{\prime}\right|^{2}+f^{\prime}\left(\mathcal{U}_{\epsilon, l}\right) \varphi^{2}+l^{2} \varphi(A+B)+\varphi O(l)\right] \mathrm{d} z=0 .
$$

Note that $\int_{0}^{1} \varphi B \mathrm{~d} z=\int_{0}^{1}\left|B^{\prime}\right|^{2} \mathrm{~d} z$ and $A=O(1 / l)$. By Proposition 9.1 we find

$$
{ }_{c 7} l^{2} \int_{0}^{1} \varphi^{2} \mathrm{~d} z \leqslant \int_{0}^{1}\left[(\epsilon / l)^{2}\left|\varphi^{\prime}\right|^{2}+f^{\prime}\left(\mathcal{U}_{\epsilon, l}\right) \varphi^{2}+l^{2} \varphi B\right] \mathrm{d} z \leqslant \int_{0}^{1}|\varphi| \mathrm{d} z O(l) .
$$

Hence

$$
\|\varphi\|_{2}=O(1 / l)
$$

Since $\left\|g_{\varepsilon}\right\|_{2}=O(1 / l)$, we conclude that $\left\|\mathcal{W}_{\epsilon, l}\right\|_{2}=O(1 / l)$. Hence $A+B=O(1 / l)$. This simplifies $(9.13)$ to

$$
-(\epsilon / l)^{2} \varphi^{\prime \prime}+f^{\prime}\left(\mathcal{U}_{\epsilon, l}\right) \varphi+O(l)=\lambda_{l} .
$$

Multiply this by $h_{\varepsilon}=(l / \epsilon) H_{\varepsilon}^{\prime}\left(l\left(z-z_{\varepsilon}\right) / \epsilon\right.$ ) (as in the proof of Proposition 9.1 , and integrate by parts:

$$
\int_{0}^{1}\left[-\varphi h_{\varepsilon}^{\prime \prime}+\varphi f^{\prime}\left(\mathcal{U}_{\epsilon, l}\right) h_{\varepsilon}\right] \mathrm{d} z=2\left(\lambda_{l}-O(l)\right)
$$

Thus by 9.14) and 9.6,

$$
2\left(\lambda_{l}-O(l)\right) \leqslant\|\varphi\|_{2}\left\|O\left(\mathrm{e}^{-C / \varepsilon}\right)+\left(f^{\prime}\left(\mathcal{U}_{\epsilon, l}\right)-f^{\prime}(H)\right) h_{\varepsilon}\right\|_{2}=O(1 / l) O\left(l^{3}\right)=O\left(l^{2}\right),
$$

which implies

$$
\lambda_{l}=O(l) .
$$

The equation (9.15) becomes

$$
-(\epsilon / l)^{2} \varphi^{\prime \prime}+f^{\prime}\left(\mathcal{U}_{\epsilon, l}\right) \varphi=O(l) .
$$

We decompose $\varphi=c h_{\varepsilon}+\varphi^{\perp}$ with $\int_{0}^{1} h_{\varepsilon} \varphi^{\perp} \mathrm{d} z=0$. By 9.14,

$$
c=\frac{\int_{0}^{1} \varphi h_{\varepsilon} \mathrm{d} z}{\left\|h_{\varepsilon}\right\|_{2}^{2}} \leqslant \frac{\|\varphi\|_{2}}{\left\|h_{\varepsilon}\right\|_{2}}=O(1) .
$$

$\varphi^{\perp}$ satisfies the equation

$$
-(\epsilon / l)^{2}\left(\varphi^{\perp}\right)^{\prime \prime}+f^{\prime}\left(\mathcal{U}_{\epsilon, l}\right) \varphi^{\perp}+c\left(f^{\prime}\left(\mathcal{U}_{\epsilon, l}\right)-f^{\prime}(H)\right) h_{\varepsilon}=O(l) .
$$

However, similarly to the argument leading to 9.6,

$$
\begin{aligned}
\left|\left(f^{\prime}\left(\mathcal{U}_{\epsilon, l}\right)-f^{\prime}(H)\right) h_{\varepsilon}\right| & \leqslant C\left|\varepsilon \phi_{0}(z)+O\left(\varepsilon^{2}\right)\right|\left|h_{\varepsilon}(z)\right| \\
& =C \varepsilon\left|\frac{\phi_{0}\left(\varepsilon t+z_{\varepsilon}\right)-\phi_{0}((1-m) / 2)}{\varepsilon}+O(1)\right| \cdot\left|H_{\varepsilon}^{\prime}(t)\right| \\
& =C \varepsilon|| t|+O(1)| \cdot\left|H_{\varepsilon}^{\prime}(t)\right|=O(\varepsilon) .
\end{aligned}
$$

So the equation for $\varphi^{\perp}$ is

$$
-(\epsilon / l)^{2}\left(\varphi^{\perp}\right)^{\prime \prime}+f^{\prime}\left(\mathcal{U}_{\epsilon, l}\right) \varphi^{\perp}=O(l) .
$$

Multiply this equation by $\varphi^{\perp}$, integrate by parts, and use 9.2 to find $\left\|\varphi^{\perp}\right\|_{2}=O(l)$. 
10. The convexity of $E$ and $E / l$

As suggested in 8.1 we define

$$
E(\epsilon, l)=\inf \left\{l J_{\epsilon, l}(\mathcal{U}): \mathcal{U} \in B_{\delta}\right\}=l J_{\epsilon, l}\left(\mathcal{U}_{\epsilon, l}\right) .
$$

Through reversal we observe

$$
E(\epsilon, l)=\inf \left\{l J_{\epsilon, l}(\mathcal{U}): \mathcal{U} \in B_{\delta}^{R}\right\}=l J_{\epsilon, l}\left(\mathcal{U}_{\epsilon, l}^{R}\right),
$$

where $\mathcal{U}_{\epsilon, l}^{R}$ is the reversal of $\mathcal{U}_{\epsilon, l}$.

Proposition 10.1 In the range $(8.3)$ both $E$ and $E / l$ are strictly convex with respect to $l$. More precisely,

$$
\begin{aligned}
\frac{\partial^{2} E}{\partial l^{2}} & =\frac{\left(1-m^{2}\right)^{2}}{4} l+O\left(\epsilon^{2 / 3}\right), \\
\frac{\partial^{2}}{\partial l^{2}}\left(\frac{E}{l}\right) & =2 c_{0}\left(\frac{\epsilon}{l^{3}}\right)+\frac{\left(1-m^{2}\right)^{2}}{12}+O\left(\epsilon^{1 / 3}\right) .
\end{aligned}
$$

Proof. Multiplying 8.6 by $\mathcal{U}_{\epsilon, l}-m$ and integrating by parts, we find the useful integral identity

$$
\int_{0}^{1}\left[(\epsilon / l)^{2}\left|\mathcal{U}_{\epsilon, l}^{\prime}\right|^{2}+f\left(\mathcal{U}_{\epsilon, l}\right)\left(\mathcal{U}_{\epsilon, l}-m\right)+l^{2}\left|\mathcal{V}_{\epsilon, l}^{\prime}\right|^{2}\right] \mathrm{d} z=0
$$

where $\mathcal{V}_{\epsilon, l}=\left(-D^{2}\right)^{-1}\left(\mathcal{U}_{\epsilon, l}-m\right)$. This identity and Lemma 8.4 turn $E$ to

$$
\begin{aligned}
E(\epsilon, l)= & l \int_{0}^{1}\left[W\left(\mathcal{U}_{\epsilon, l}\right)-\frac{f\left(\mathcal{U}_{\epsilon, l}\right)\left(\mathcal{U}_{\epsilon, l}-m\right)}{2}\right] \mathrm{d} z \\
= & \epsilon \int_{-\infty}^{\infty}\left(W(H)-\frac{f(H)(H-m)}{2}\right) \mathrm{d} t \\
& +\epsilon \int_{0}^{1}\left(f( \pm 1)-\frac{f^{\prime}( \pm 1)( \pm 1-m)+f( \pm 1)}{2}\right) \phi_{0} \mathrm{~d} z+O\left(\epsilon^{5 / 3}\right) \\
= & c_{0} \epsilon+\frac{\left(1-m^{2}\right)^{2}}{24} l^{3}+O\left(\epsilon^{5 / 3}\right) .
\end{aligned}
$$

The integral in the second line is $c_{0}$ because of 2.3 and 2.4 . The computation of the integral in the third line uses the definition 8.10 ) of $\phi_{0}$ and the expression 3.4 of $\mathcal{V}_{0}$.

Differentiating $E$ with respect to $l$ yields

$$
\frac{\partial E}{\partial l}=\int_{0}^{1}\left[-\frac{\epsilon^{2}}{2 l^{2}}\left|\mathcal{U}_{\epsilon, l}^{\prime}\right|^{2}+W\left(\mathcal{U}_{\epsilon, l}\right)+\frac{3 l^{2}}{2}\left|\mathcal{V}_{\epsilon, l}^{\prime}\right|^{2}\right] \mathrm{d} z .
$$

We have used the fact that $\mathcal{U}_{\epsilon, l}$ is a critical point of $J_{\epsilon, l}$. By 10.1 and Lemma 8.4 this becomes

$$
\begin{aligned}
\frac{\partial E}{\partial l}= & \int_{0}^{1}\left[W\left(\mathcal{U}_{\epsilon, l}\right)+\frac{f\left(\mathcal{U}_{\epsilon, l}\right)\left(\mathcal{U}_{\epsilon, l}-m\right)}{2}\right] \mathrm{d} z+\int_{0}^{1} 2 l^{2}\left|\mathcal{V}_{\epsilon, l}^{\prime}\right|^{2} \mathrm{~d} z \\
= & \frac{\epsilon}{l} \int_{-\infty}^{\infty}\left[W(H)+\frac{f(H)(H-m)}{2}\right] \mathrm{d} t+\frac{\epsilon}{l} \int_{0}^{1} \frac{f^{\prime}( \pm 1)( \pm 1-m)}{2} \phi_{0} \mathrm{~d} z \\
& +\int_{0}^{1} 2 l^{2}\left|\mathcal{V}_{\epsilon, l}^{\prime}\right|^{2} \mathrm{~d} z+O\left(\epsilon^{4 / 3}\right) .
\end{aligned}
$$


The first integral in the second line is 0 again by $(2.3)$ and $(2.4)$. The second integral equals $-\left(1-m^{2}\right)^{2} / 24$ multiplied by $l^{2} / \epsilon$ as in the estimate of $E$. To estimate the integral in the last line note (8.17), so using (3.4) we deduce

$$
\int_{0}^{1} 2 l^{2}\left|\mathcal{V}_{\epsilon, l}^{\prime}\right|^{2} \mathrm{~d} z=\int_{0}^{1} 2 l^{2}\left|\mathcal{V}_{0}^{\prime}\right|^{2} \mathrm{~d} z+O\left(\epsilon^{4 / 3}\right)=\frac{\left(1-m^{2}\right)^{2}}{6} l^{2}+O\left(\epsilon^{4 / 3}\right) .
$$

Altogether

$$
\frac{\partial E}{\partial l}=\frac{\left(1-m^{2}\right)^{2}}{8} l^{2}+O\left(\epsilon^{4 / 3}\right)
$$

Differentiate (10.3) with respect to $l$. Denote the derivative of $\mathcal{U}_{\epsilon, l}$ with respect to $l$ by $\mathcal{W}_{\epsilon, l}$ and the derivative of $\mathcal{V}_{\epsilon, l}$ with respect to $l$ by $\mathcal{Z}_{\epsilon, l}$. Then

$$
\frac{\partial^{2} E}{\partial l^{2}}=\int_{0}^{1}\left[\frac{\epsilon^{2}}{l^{3}}\left|\mathcal{U}_{\epsilon, l}^{\prime}\right|^{2}+\left.3 l|| \mathcal{V}_{\epsilon, l}^{\prime}\right|^{2}\right] \mathrm{d} z+\int_{0}^{1}\left[-\frac{\epsilon^{2}}{l^{2}} \mathcal{U}_{\epsilon, l}^{\prime} \mathcal{W}_{\epsilon, l}^{\prime}+f\left(\mathcal{U}_{\epsilon, l}\right) \mathcal{W}_{\epsilon, l}+3 l^{2} \mathcal{V}_{\epsilon, l}^{\prime} \mathcal{Z}_{\epsilon, l}^{\prime}\right] \mathrm{d} z .
$$

Call the first term on the right $T_{1}$ and the second term $T_{2}$. The estimation of $T_{1}$ is similar to the earlier ones. Using (10.1) we find

$$
\begin{aligned}
T_{1} & =\frac{1}{l} \int_{0}^{1}\left[-f\left(\mathcal{U}_{\epsilon, l}\right)\left(\mathcal{U}_{\epsilon, l}-m\right)+2 l^{2}\left|\mathcal{V}_{\epsilon, l}^{\prime}\right|^{2}\right] \mathrm{d} z \\
& =\frac{\epsilon}{l^{2}} \int_{-\infty}^{\infty}-f(H)(H-m) \mathrm{d} t+\frac{\left(1-m^{2}\right)^{2}}{12} l+\frac{\left(1-m^{2}\right)^{2}}{6} l+O(\epsilon) \\
& =\frac{\epsilon}{l^{2}} \int_{-\infty}^{\infty}-f(H) H \mathrm{~d} t+\frac{\left(1-m^{2}\right)^{2}}{4} l+O(\epsilon) .
\end{aligned}
$$

To estimate $T_{2}$, first use 8.6 and $\mathcal{Z}_{\epsilon, l}=\left(-D^{2}\right)^{-1} \mathcal{W}_{\epsilon, l}$ to simplify it to

$$
T_{2}=\int_{0}^{1}\left[2 f\left(\mathcal{U}_{\epsilon, l}\right) \mathcal{W}_{\epsilon, l}+4 l^{2} \mathcal{V}_{\epsilon, l} \mathcal{W}_{\epsilon, l}\right] \mathrm{d} z
$$

By Propositions 8.3 and 9.3

$$
\begin{aligned}
T_{2} & =\int_{0}^{1}\left[2 f(H)+O\left(\epsilon^{2 / 3}\right)\right]\left(\frac{z-z_{\varepsilon}}{\epsilon} H^{\prime}+\phi^{\perp}+O\left(\epsilon^{1 / 3}\right)\right) \mathrm{d} z \\
& =\frac{\epsilon}{l^{2}} \int_{-z_{\varepsilon} / \varepsilon}^{\left(1-z_{\varepsilon}\right) / \varepsilon} 2 f(H(t)) H^{\prime}(t) t \mathrm{~d} t+O\left(\epsilon^{2 / 3}\right)=\frac{\epsilon}{l^{2}} \int_{-\infty}^{\infty}-2 W(H) \mathrm{d} t+O\left(\epsilon^{2 / 3}\right) .
\end{aligned}
$$

We have used the estimates

$$
\begin{gathered}
\int_{0}^{1}\left|\frac{z-z_{\varepsilon}}{\epsilon} H^{\prime}\right| \mathrm{d} z=\frac{\epsilon}{l^{2}} \int_{-z_{\varepsilon} / \varepsilon}^{\left(1-z_{\varepsilon}\right) / \varepsilon}\left|H^{\prime}(t) t\right| \mathrm{d} t=O\left(\epsilon^{1 / 3}\right), \\
\int_{0}^{1}|f(H)| \mathrm{d} z=\frac{\epsilon}{l} \int_{-z_{\varepsilon} / \varepsilon}^{\left(1-z_{\varepsilon}\right) / \varepsilon}|f(H(t))| \mathrm{d} t=O\left(\epsilon^{2 / 3}\right), \\
\left\|2 f(H)+O\left(\epsilon^{2 / 3}\right)\right\|_{2}=O\left(\epsilon^{1 / 3}\right) .
\end{gathered}
$$


Adding $T_{1}$ and $T_{2}$, since $\int_{-\infty}^{\infty}(f(H) H+2 W(H)) \mathrm{d} t=0$ as in the estimation for $\partial E / \partial l$, we arrive at

$$
\frac{\partial^{2} E}{\partial l^{2}}=\frac{\left(1-m^{2}\right)^{2}}{4} l+O\left(\epsilon^{2 / 3}\right),
$$

proving the estimate for $\partial^{2} E / \partial l^{2}$. From $10.2, \sqrt{10.4}$ and 10.5 , we deduce

$$
\frac{\partial^{2}}{\partial l^{2}}\left(\frac{E}{l}\right)=\frac{\frac{\partial^{2} E}{\partial l^{2}} l^{2}-2 \frac{\partial E}{\partial l} l+2 E}{l^{3}}=2 c_{0}\left(\frac{\epsilon}{l^{3}}\right)+\frac{\left(1-m^{2}\right)^{2}}{12}+O\left(\epsilon^{1 / 3}\right) .
$$

We now prove the three theorems stated in Section 1. Recall that a global minimum of $I_{\epsilon}$ is denoted by $u_{\epsilon}$, with $N_{\epsilon} \alpha$-points, denoted by $x_{1}, \ldots, x_{N_{\epsilon}}$. Between them there are $N_{\epsilon}-1$ zeros of the derivative of $v_{\epsilon}=\left(-D^{2}\right)^{-1}\left(u_{\epsilon}-m\right)$, denoted by $y_{1}, \ldots, y_{N_{\epsilon}-1}$, satisfying $0<x_{1}<y_{1}<$ $x_{2}<y_{2}<\ldots<x_{N_{\epsilon}-1}<y_{N_{\epsilon}-1}<x_{N_{\epsilon}}<1$. We set $l_{i}=y_{i}-y_{i-1}$ for $i=1, \ldots, N_{\epsilon}$ with $y_{0}=0$ and $y_{N_{\epsilon}}=1$. There are two possibilities for $u_{\epsilon}$ on $\left(0, x_{1}\right): u_{\epsilon}>\alpha$ or $u_{\epsilon}<\alpha$.

Proof of Theorem 1.1 Without loss of generality we suppose that $u_{\epsilon}>\alpha$ on $\left(0, x_{1}\right)$. We construct a particular periodic solution $u_{\epsilon}^{*}$ with $N_{\epsilon} \alpha$-points (i.e. $N_{\epsilon} / 2$ periods), and show that $u_{\epsilon}=u_{\epsilon}^{*}$.

Let $\mathcal{U}_{\epsilon, 1 / N_{\epsilon}}$ be the unique minimum of $J_{\epsilon, l}$ in $B_{\delta}$ (Proposition 9.2), with $l=1 / N_{\epsilon}$, and let $\mathcal{U}_{\epsilon, 1 / N_{\epsilon}}^{R}$, its reversal, be the unique minimum of $J_{\epsilon, 1 / N_{\epsilon}}$ in $B_{\delta}^{R}$. Set $u_{\epsilon}^{*}(x)=\mathcal{U}_{\epsilon, 1 / N_{\epsilon}}^{R}\left(N_{\epsilon} x\right)$ for $x \in\left(0,1 / N_{\epsilon}\right)$. Extend $u_{\epsilon}^{*}$ anti-periodically to $(0,1)$, i.e. $u_{\epsilon}^{*}(x)=\mathcal{U}_{\epsilon, 1 / N_{\epsilon}}\left(N_{\epsilon} x-1\right)$ for $x \in\left(1 / N_{\epsilon}, 2 / N_{\epsilon}\right), u_{\epsilon}^{*}(x)=\mathcal{U}_{\epsilon, 1 / N_{\epsilon}}^{R}\left(N_{\epsilon} x-2\right)$ for $x \in\left(2 / N_{\epsilon}, 3 / N_{\epsilon}\right)$, etc. Clearly $u_{\epsilon}^{*}$ is periodic with $N_{\epsilon} / 2$ periods.

For small $\epsilon$ by Lemma 4.6 and Propositions 7.1 and 7.2, $u_{\epsilon}\left(l_{i} \cdot+y_{i-1}\right) \in B_{\delta}$ when $i$ is even, and $\in B_{\delta}^{R}$ when $i$ is odd. Using the strict convexity of $E$ in Proposition 10.1 and 8.1 we find

$$
I_{\epsilon}\left(u_{\epsilon}^{*}\right) \geqslant I_{\epsilon}\left(u_{\epsilon}\right) \geqslant \sum_{i=1}^{N_{\epsilon}} l_{i} J_{\epsilon, l_{i}}\left(u_{\epsilon}\left(l_{i} \cdot+y_{i-1}\right)\right) \geqslant \sum_{i=1}^{N_{\epsilon}} E\left(\epsilon, l_{i}\right) \geqslant N_{\epsilon} E\left(\epsilon, 1 / N_{\epsilon}\right)=I_{\epsilon}\left(u_{\epsilon}^{*}\right) .
$$

All the inequalities above must be equalities. Therefore $l_{i}=1 / N_{\epsilon}$ for all $i$, and $u_{\epsilon}\left(l_{i} \cdot+y_{i-1}\right)=$ $\mathcal{U}_{\epsilon, 1 / N_{\epsilon}}$ when $i$ is even, and $=\mathcal{U}_{\epsilon, 1 / N_{\epsilon}}^{R}$ when $i$ is odd, by Proposition 9.2. Thus $u_{\epsilon}=u_{\epsilon}^{*}$.

If on $\left(0, x_{1}\right), u_{\epsilon}<\alpha$, then $u_{\epsilon}$ must be the reversal of $u_{\epsilon}^{*}$.

Proof of Theorem 1.2 In the previous proof we have shown that if $N_{\epsilon}$ is known, there are exactly two global minima of $I_{\epsilon}, u_{\epsilon}^{*}$ and its reversal, with $N_{\epsilon} \alpha$-points. Here we determine whether $N_{\epsilon}$ is unique.

By the strict convexity of $E / l$ (Proposition 10.1), $E / l$ attains its minimum at a unique $l_{*}$. But for $I_{\epsilon}\left(u_{\epsilon}\right)=N_{\epsilon} E\left(\epsilon, 1 / N_{\epsilon}\right)$, its minimum with respect to $N_{\epsilon}$ is achieved at one or two integers.

If $1 / l_{*}$ happens to be an integer, then there is only one $N_{\epsilon}=1 / l_{*}$. If $1 / l_{*}$ is not an integer, there exist two consecutive integers, say $N$ and $N+1$, such that $N<1 / l_{*}<N+1$. If $N E(\epsilon, 1 / N) \neq$ $(N+1) E(\epsilon, 1 /(N+1))$, then again there is only one $N_{\epsilon}$. It must be the one of $N$ and $N+1$ which offers the smaller of $N E(\epsilon, 1 / N)$ and $(N+1) E(\epsilon, 1 /(N+1))$. In these two cases we have two global minima of $I_{\epsilon}$.

In the less likely third case that $1 / l_{*}$ is not an integer, and $N E(\epsilon, 1 / N)=(N+1) E(\epsilon, 1 /(N+1))$, we have two values, $N$ and $N+1$, for $N_{\epsilon}$. Then there are four global minima of $I_{\epsilon}$.

Proof of Theorem 1.3 Collecting the estimates 10.2 and 10.4 , we have

$$
\frac{\partial}{\partial l}\left(\frac{E}{l}\right)=\frac{\frac{\partial E}{\partial l} l-E}{l^{2}}=\frac{-c_{0} \epsilon+\frac{\left(1-m^{2}\right)^{2}}{12} l^{3}+O\left(\epsilon^{5 / 3}\right)}{l^{2}} .
$$


If $E / l$ is minimized at $l=l_{*}$, the above estimate implies

$$
-c_{0} \epsilon+\frac{\left(1-m^{2}\right)^{2}}{12} l_{*}^{3}+O\left(\epsilon^{5 / 3}\right)=0,
$$

which in turn yields

$$
l_{*}=\left(\frac{12 c_{0}}{\left(1-m^{2}\right)^{2}}\right)^{1 / 3} \epsilon^{1 / 3}+O(\epsilon) .
$$

$N_{\epsilon}$, the number of $\alpha$-points of $u_{\epsilon}$, is either $1 / l_{*}$ if it happens to be an integer, or one of the two consecutive integers, $N$ and $N+1$, such that $1 /(N+1)<l_{*}<1 / N$. In the first case the theorem is proved since $2 l_{*}=2 / N_{\epsilon}$ is the period. In the second case, since

$$
\frac{1}{N}-\frac{1}{N+1}<\frac{l_{*}^{2}}{1-l_{*}}=O\left(\epsilon^{2 / 3}\right),
$$

the period $2 / N$ or $2 /(N+1)$ of $u_{\epsilon}$ is $2 l_{*}+O\left(\epsilon^{2 / 3}\right)$, proving the theorem by 10.6 .

\section{Acknowledgments}

Some of the ideas in this paper emerged during our several stays in 1997 and 98 at Mathematisches Forschungsinstitut Oberwolfach, which we would like to thank for its hospitality. The first author also thanks the Department of Mathematics and the Institute of Mathematical Sciences at the Chinese University of Hong Kong where he spent several crucial weeks during the course of this work.

\section{REFERENCES}

1. Carr, J., Gurtin, M. E., \& Slemrod, M. Structured phase transitions on a finite interval. Arch. Rational Mech. Anal. 86 (1984), 317-351. Zbl 0564.76075 MR 86i:80001

2. CHOKSI, R. Scaling laws in microphase separation of diblock copolymers. J. Nonlinear Sci. 11 (2001), 223-236. Zbl pre01709140 MR 1852942

3. Fife, P. C. \& Hilhorst, D. The Nishiura-Ohnishi free boundary problem in the 1D case. SIAM J. Math. Anal. 33 (2001), 589-606. Zbl pre01670366 MR 2003a:35207

4. Henry, M. Singular limit of a fourth-order problem arising in the micro-phase separation of diblock copolymers. Adv. Differential Equations 6 (2001), 1049-1114. Z Zbl pre01700808 MR 2002k:35027

5. MÜLLER, S. Singular perturbations as a selection criterion for periodic minimizing sequences. Calc. Var. Partial Differential Equations 1 (1993), 169-204. Zbl 0821.49015 MR 95k:49030

6. Muratov, C. B. Theory of domain patterns in systems with long-range interactions of Coulomb type. Preprint.

7. Ni, W.-M. \& TAKAGI, I. On the shape of least energy solution to a semilinear Neumann problem. Comm. Pure Appl. Math. 41 (1991), 819-851. Zbl 0754.35042 MR 92i:35052

8. NI, W.-M. \& TAKAGI, I. Locating the peaks of least energy solutions to a semilinear Neumann problem. Duke Math. J. 70 (1993), 247-281. Zbl 0796.35056 MR 94h:35072

9. NI, W.-M. \& WEI, J. On the location and profile of spike-layer solutions to singularly perturbed semilinear Dirichlet problems. Comm. Pure Appl. Math. 48 (1995), 723-761. Zbl 0838.35009 MR 96g:35077 
10. NishiurA, Y. \& OHNishi, I. Some mathematical aspects of the microphase separation in diblock copolymers. Phys. D 84 (1995), 31-39.

11. Ohnishi, I., Nishiura, Y., Imai, M., \& Matsushita, Y. Analytical solutions describing the phase separation driven by a free energy functional containing a long-range interaction term. Chaos 9 (1999), 329-341. Zbl 0970.35151 MR 2000d:35244

12. Ohta, T. \& KAWASAKI, K. Equilibrium morphology of block copolymer melts. Macromolecules 19 (1986), 2621-2632.

13. REN, X. \& WEI, J. On the multiplicity of solutions of two nonlocal variational problems. SIAM J. Math. Anal. 31 (2000), 909-924. Zbl 0973.49007 MR 2001a:74035

14. REN, X. \& WEI, J. Concentrically layered energy equilibria of the di-block copolymer problem. European J. Appl. Math. 31 (2002), 479-496. MR 1939157

15. REN, X. \& WEI, J. Triblock copolymer theory: Free energy, disordered phase and weak segregation. Phys. $D$, to appear.

16. REN, X. \& WEI, J. Triblock copolymer theory: Ordered ABC lamellar phase. J. Nonlinear Sci., to appear.

17. REN, X. \& WEI, J. On the spectra of 3-D lamellar solutions of the diblock copolymer problem. SIAM J. Math. Anal., to appear.

18. REN, X. \& WEI, J. Wriggled lamellar solutions and their stability in the diblock copolymer problem. Preprint.

19. Truskinovsky, L. \& ZANZOtTo, G. Finite scale microstructures and metastability in one dimensional elasticity. Meccanica 30 (1995), 577-589. MR 96h:73012

20. Truskinovsky, L. \& Zanzotto, G. Ericksen's bar revisited: Energy wiggles. J. Mech. Phys. Solids 44 (1996), 1371-1408. MR 97e:73015

21. WeI, J. Conditions for two-peaked solutions of singularly perturbed elliptic equations. Manuscripta Math. 96 (1998), 113-131. Zbl 0901.35003 MR 99e:35014 